\title{
A Study of Selected Methods of Nonparametric Regression Estimation
}

\author{
by
}

\section{Oksana Chkrebtii, B. Math (Hons.)}

\author{
A Thesis submitted to \\ the Faculty of Graduate Studies and Research \\ in partial fulfillment of \\ the requirements for the degree of \\ Master of Science
}

Ottawa-Carleton Institute for

Mathematics and Statistics

School of Mathematics and Statistics

Carleton University

Ottawa, Ontario, Canada

May 2008

(c) Copyright

2008 - Oksana Chkrebtii 


$\begin{array}{ll}\begin{array}{l}\text { Library and } \\ \text { Archives Canada }\end{array} & \begin{array}{l}\text { Bibliothèque et } \\ \text { Archives Canada }\end{array} \\ \begin{array}{l}\text { Published Heritage } \\ \text { Branch }\end{array} & \begin{array}{l}\text { Direction du } \\ \text { Patrimoine de l'édition }\end{array} \\ \begin{array}{l}\text { 395 Wellington Street } \\ \text { Ottawa ON K1A 0N4 } \\ \text { Canada }\end{array} & \begin{array}{l}\text { 395, rue Wellington } \\ \text { Ottawa ON K1A 0N4 } \\ \text { Canada }\end{array}\end{array}$

Your file Votre référence ISBN: 978-0-494-44118-3 Our file Notre référence ISBN: 978-0-494-44118-3

NOTICE:

The author has granted a nonexclusive license allowing Library and Archives Canada to reproduce, publish, archive, preserve, conserve, communicate to the public by telecommunication or on the Internet, loan, distribute and sell theses worldwide, for commercial or noncommercial purposes, in microform, paper, electronic and/or any other formats.

The author retains copyright ownership and moral rights in this thesis. Neither the thesis nor substantial extracts from it may be printed or otherwise reproduced without the author's permission.
AVIS:

L'auteur a accordé une licence non exclusive permettant à la Bibliothèque et Archives Canada de reproduire, publier, archiver, sauvegarder, conserver, transmettre au public par télécommunication ou par l'Internet, prêter, distribuer et vendre des thèses partout dans le monde, à des fins commerciales ou autres, sur support microforme, papier, électronique et/ou autres formats.

L'auteur conserve la propriété du droit d'auteur et des droits moraux qui protège cette thèse. $\mathrm{Ni}$ la thèse ni des extraits substantiels de celle-ci ne doivent être imprimés ou autrement reproduits sans son autorisation.
In compliance with the Canadian Privacy Act some supporting forms may have been removed from this thesis.

While these forms may be included in the document page count, their removal does not represent any loss of content from the thesis.
Conformément à la loi canadienne sur la protection de la vie privée, quelques formulaires secondaires ont été enlevés de cette thèse.

Bien que ces formulaires aient inclus dans la pagination, il n'y aura aucun contenu manquant.

\section{Canada}




\begin{abstract}
Nonparametric regression methods are widely used to estimate an unknown regression function from noisy data. In this thesis, some topics in nonparametric regression analysis are investigated in detail and then applied to simulated and actual medical data under a fixed design model. The topics under study include kernel estimation, orthogonal series estimation and spline smoothing. The accuracy of a nonparametric estimator crucially depends on the choice of the so-called "smoothing parameter". The adaptive methods of cross-validation and generalized cross-validation for choosing the smoothing parameter are considered. The performance of various regression estimators and their relationship to each other are discussed, and this discussion is illustrated with simulations.
\end{abstract}




\section{Acknowledgements}

I would like to thank all the people who have helped and inspired me during my time as a Master's student at the School of Mathematics and Statistics. I would especially like to thank my supervisor, Dr. Natalia Stepanova, for her guidance and support during this research. Her knowledge and valuable advice have been essential throughout. I would also like to thank Drs. Raluca Balan, Jason Nielsen and Michiel Smid for acting on my graduate committee.

I am especially grateful to my family for their love and encouragement. They have always been the best of role models, and were always there for me. 


\section{Contents}

1 Introduction and Review of Literature 1

1.1 Statement of the Problem . . . . . . . . . . . . . . . 1

1.2 Fixed Design Regression Model ... . . . . . . . . . . . . 2

1.3 Nonparametric Regression and Review of Literature . . . . . . . . . . 3

1.4 Measures of Fit . . . . . . . . . . . . . . . 8

1.5 Linear Estimators . . . . . . . . . . . . . . . . 10

2 Kernel Regression Estimation 12

2.1 Kernel Functions . . . . . . . . . . . . . . . . . . . 14

2.2 Kernel Regression Estimators . . . . . . . . . . . . . . . 17

2.3 Asymptotic Properties . . . . . . . . . . . . . . . 25

3 Orthogonal Series Estimation $\quad 33$

3.1 Some Function Space Theory . . . . . . . . . . . . . . . 33

3.2 Two Types of Design and General Form of Projection Estimators . . 37

3.3 Projection Estimators Corresponding to Different Designs . . . . . . . 44

3.4 Relationship to Kernel Regression Estimators . . . . . . . . . . . . . 62

4 Spline Smoothing $\quad 67$

4.1 Definition and Basic Properties of Spline Functions . . . . . . . . . 67

4.2 Spline Smoothing Estimators . . . . . . . . . . . . . 71

4.3 Relationship to Other Regression Estimators . . . . . . . . . . . . . . 87

4.4 Asymptotic Performance . . . . . . . . . . . . . 91

5 Choice of Smoothing Parameter $\quad 93$

6 Conclusion $\quad 111$ 


\section{List of Tables}

1 Some standard kernel functions and their Fourier transforms. . . . . . . . . . . 14

2 Some non-standard kernel functions and their Fourier transforms. . . . . . . . . 15

3 Liver Function Test Data. . . . . . . . . . . . . . . . . . 77

\section{List of Figures}

1 Some well-known kernels. . . . . . . . . . . . . . . . . . 15

2 Some uncommon kernel functions . . . . . . . . . . . . . . . 16

3 The kernel regression estimator $f_{2 n+1}(x)=\frac{1}{2 n+1} \sum_{i=-n}^{n} K_{h}\left(x-x_{i}\right) Y_{i}$ is shown (dotted line), where $K_{h}(\cdot)$ is a standard kernel. The estimator is based on $2 n+1=$ 40 data points from $Y_{i}=f\left(x_{i}\right)+\varepsilon_{i}$, where $x_{i}=\frac{2 i}{2 n+1}, i=-n, \ldots,-1,0,1, \ldots, n$. The regression function is given by $f(x)=-0.95 \cos ^{3}(2 \pi x+1.6 \pi)$ (solid line) and the error terms $\varepsilon_{1}, \ldots, \varepsilon_{n}$ are randomly generated from a normal distribution with mean 0 and standard deviation 0.2. The smoothing parameters $h_{C V}$ and $h_{G C V}$ were obtained by the methods of cross-validation, and generalized cross-validation respectively (see Chapter 5 for details). . . . . . . . . . . . . . . .

4 The kernel regression estimator $f_{2 n+1}(x)=\frac{1}{2 n+1} \sum_{i=1}^{n} K_{h}\left(x-x_{i}\right) Y_{i}$ is shown (dotted line), where $K_{h}(\cdot)$ is a non-standard kernel. The estimator is based on $2 n+1=40$ data points from $Y_{i}=f\left(x_{i}\right)+\varepsilon_{i}$, where $x_{i}=\frac{2 i}{2 n+1}, i=$ $-n, \ldots,-1,0,1, \ldots, n$. The regression function is given by $f(x)=-0.95 \cos ^{3}(2 \pi x+1.6 \pi)$ (solid line) and the error terms $\varepsilon_{1}, \ldots, \varepsilon_{n}$ are randomly generated from a normal distribution with mean 0 and standard deviation 0.2. The smoothing parameters $h_{C V}$ and $h_{G C V}$ were obtained by the methods of cross-validation, and generalized cross-validation respectively (see Chapter 5 for details). . . . . . . . . . . .

5 Chebyshev design on the interval $[-1,1]$ is equivalent to the projection on the $x$ axis of $n$ points that are equally-spaced on the unit semi-circle centered at the point

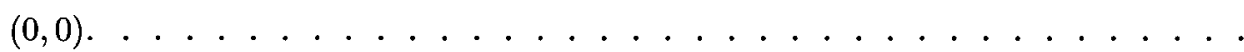

6 The regression estimator $\tilde{f}_{n, 1}(x)=\sum_{k=0}^{N-1} \tilde{d}_{k} q_{k}(x)$ (dotted line) is shown. It is based on $n=20$ data points from $Y_{i}=f\left(x_{i}\right)+\varepsilon_{i}$, where $x_{i}=\frac{2 i-1}{2 n}$. The regression function is given by $f(x)=-0.95 \cos ^{3}(2 \pi x+1.6 \pi)$ (solid line) and the error terms $\varepsilon_{1}, \ldots, \varepsilon_{n}$ are randomly generated from a normal distribution with mean 0 and standard deviation 0.2. The smoothing parameter values, $N_{C V}=5$ and $N_{G C V}=5$, were obtained by the methods of cross-validation and generalized cross-validation, respectively (see Chapter 5 for details). . . . . . . . . . . . . . . . 
7 The regression estimator $\tilde{f}_{n, 2}(x)=\sum_{k=0}^{N-1} \tilde{a}_{k} h_{k}(x)$ (dotted line) is shown. It is based on $n=40$ data points from $Y_{i}=f\left(x_{i}\right)+\varepsilon_{i}$, where $x_{i}=\frac{2 i}{2 n+1}$. The regression function is given by $f(x)=-0.95 \cos ^{3}(2 \pi x+1.6 \pi)$ (solid line) and the error terms $\varepsilon_{1}, \ldots, \varepsilon_{n}$ are randomly generated from a normal distribution with mean 0 and standard deviation 0.2 . The smoothing parameters $N_{C V}=7$ and $N_{G C V}=9$ were obtained by the methods of cross-validation and generalized crossvalidation, respectively (see Chapter 5 for details). . . . . . . . . . .

8 The regression estimator $\tilde{f}_{n, 3}(x)=\sum_{k=0}^{N-1} \tilde{b}_{k} t_{k}(x)$ (dotted line) is shown. It is based on $n=40$ data points from $Y_{i}=f\left(x_{i}\right)+\varepsilon_{i}$, where $x_{i}=\frac{(2 i-1) \pi}{2 n}$. The regression function is given by $f(x)=-0.95 \cos ^{3}(2 \pi x+1.6 \pi)$ (solid line) and the error terms $\varepsilon_{1}, \ldots, \varepsilon_{n}$ are randomly generated from a normal distribution with mean 0 and standard deviation 0.2. The smoothing parameters $N_{C V}=10$ and $N_{G C V}=10$ were obtained by the methods of cross-validation and generalized cross-validation, respectively (see Chapter 5 for details). . . . . . . . . . .

9 Cosine Fourier transforms of the uniform and Epanechnikov kernels (kernels with support on $[-1,1].) \ldots \ldots \ldots \ldots \ldots \ldots$

10 A cubic spline $s$ (thick solid line), its first derivative $s^{\prime}$ (thin solid line) and its second derivative $s^{\prime \prime}$ (dashed line) with respect to $\{0,2,4,6,8,10\}$. . . . . . .

11 A cubic smoothing spline (dotted line) fit to $n=40$ data points from $Y_{i}=f\left(x_{i}\right)+\varepsilon_{i}$, where $\left\{x_{i}\right\}_{i=1}^{n}$ follows the Chebyshev design. The regression function is $f(x)=$ $-0.95 \cos ^{3}(2 \pi x+1.6 \pi)$ (solid line) and the error terms $\varepsilon_{1}, \ldots, \varepsilon_{n}$ are randomly generated from a normal distribution with mean 0 and standard deviation 0.2 . The spline smoother was produced by the function smooth.spline() in S-Plus. . .

12 Liver function test scores $\left(X_{i}\right)$ versus survival times $\left(Y_{i}\right)$ of liver patients. The data set was obtained from the paper by Shih and Weisberg (1986). The data has several replications (more than one response value for a single value $X_{i}$ ). For the purpose of spline smoothing, the replicate response were averaged for every level $X_{i} .78$

13 Liver function test scores $\left(X_{i}\right)$ versus survival times $\left(Y_{i}\right)$ of liver patients with weighted smoothing spline estimators (based on the averaged responses for every $X_{i}$ with weights $\left.w_{i}=n_{i}, i=1, \ldots, m\right)$. The smoothing parameters were chosen by the methods of cross-validation (estimator as solid line, $\lambda_{C V}=0.0019944384$ ) and generalized cross-validation (estimator as dotted line, $\lambda_{C V}=0.003827022$ ). .

14 Residual plot for weighted smoothing spline estimator (based on the averaged responses for every $X_{i}$ with weights $w_{i}=n_{i}, i=1, \ldots, m$ ) with smoothing parameter $\lambda_{C V}=0.0019944384 \ldots \ldots \ldots \ldots \ldots \ldots \ldots$

15 QQ-plot for weighted smoothing spline estimator (based on the averaged responses for every $X_{i}$ with weights $\left.w_{i}=n_{i}, i=1, \ldots, m\right)$ with smoothing parameter $\lambda_{C V}=$ $0.0019944384 \ldots \ldots \ldots \ldots \ldots \ldots \ldots$

16 Spline kernel function $K_{s}(u)=\frac{1}{2} \exp \left(-\frac{|u|}{\sqrt{2}}\right) \sin \left(\frac{|u|}{\sqrt{2}}+\frac{\pi}{4}\right) \ldots \ldots \ldots \ldots$ 
17 A cubic smoothing spline (solid line) fit to $n=50$ data points using model $Y_{i}=f\left(x_{i}\right)+\varepsilon_{i}$, where $\left\{x_{i}\right\}_{i=1}^{50}$ is a uniform design. The regression function is $f(x)=\cos ^{3}(2 \pi x)$ and the error terms $\varepsilon_{1}, \ldots, \varepsilon_{n}$ are randomly generated from an $N\left(0,(0.15)^{2}\right)$ normal distribution. The spline smoother was produced by the function smooth.spline() in S-Plus with smoothing parameter $\lambda$ chosen by the methods of cross-validation and generalized cross-validation, respectively. An equivalent spline kernel fit to the data (dotted line) uses a kernel regression estimator with the spine kernel $K_{s}$ and a bandwidth of $\left(n^{-1} \lambda\right)^{1 / 4}$, where $\lambda=\lambda_{C V}$ and $\lambda=\lambda_{G C V} . \quad 90$ 


\title{
Symbols and Notation
}

\section{Estimators}

\author{
$f(x)$ \\ $f_{n}(x)$ \\ $f_{n,-i}(x)$ \\ regression function evaluated at the point $x$ \\ estimator of regression function evaluated at the \\ point $x$ \\ leave-one-out estimator of regression function evalu- \\ ated at the point $x$
}

\section{Function Spaces}

$$
\begin{aligned}
& L_{2}[a, b] \\
& C^{m}[a, b] \\
& C^{\infty}[a, b] \\
& W_{2}^{m}[a, b] \\
& \mathcal{P}^{r}[a, b] \\
& S^{r}\left(x_{0}, \ldots, x_{n+1}\right) \\
& \text { the space of splines of degree } r \text { on the interval }[a, b] \\
& \text { with respect to design points } x_{1}, \ldots, x_{n} \text { with } x_{0}=a \\
& \text { and } x_{n+1}=b \\
& N S^{2 m-1}\left(x_{0}, \ldots, x_{n+1}\right) \quad \text { the space of natural splines of degree } 2 m-1 \text { on the } \\
& \text { interval }[a, b] \text { with respect to design points } x_{1}, \ldots, x_{n} \\
& \text { with } x_{0}=a \text { and } x_{n+1}=b
\end{aligned}
$$




\section{Abbreviations}

$P\left(f_{n}, f\right)$ or $P(h) \quad$ prediction risk

$R S S\left(f_{n}, f\right)$ or $R S S(h) \quad$ regression sum of squares

$R\left(f_{n}, f\right)$ or $R(h) \quad$ unbiased risk

$C V(h)$

cross-validation score

$G C V(h)$

generalized cross-validation score

\section{Convergence}

$\begin{array}{ll}f=o(g) & \text { "small oh" symbol means: } f(x) / g(x) \rightarrow 0 \text { as } x \rightarrow a \\ f=O(g) & \text { "big oh" symbol means: there exists a constant } C> \\ & 0 \text { such that } 0<|f(x) / g(x)|<C \text { as } x \rightarrow a \\ & \\ & \text { "small oh-P" symbol means: suppose that }\left\{X_{n}\right\} \text { and } \\ & \left\{Y_{n}\right\} \text { are two sequences of random variables, then } \\ & X_{n}=o_{P}\left(Y_{n}\right) \text { if for any } \varepsilon>0, \mathbf{P}\left(\left|X_{n} / Y_{n}\right|>\varepsilon\right) \rightarrow 0 \\ & \text { as } n \rightarrow \infty \\ & \text { "big oh-P" symbol means: suppose that }\left\{X_{n}\right\} \text { and } \\ & \left\{Y_{n}\right\} \text { are two sequences of random variables, then } \\ & X_{n}=O_{P}\left(Y_{n}\right) \text { if for any } \varepsilon>0 \text { there exists } c>0 \\ & \text { such that } \mathbf{P}\left(\left|X_{n} / Y_{n}\right|>c\right)<\varepsilon \text { as } n \rightarrow \infty \\ & \\ & \text { convergence in probability }\end{array}$




\section{Introduction and Review of Literature}

\subsection{Statement of the Problem}

A common problem in statistics is to find the relationship between a predictor and a response variable in the presence of a random error, or "noise". This problem appears in many fields including economics, biology and the social sciences. A common analogy is a radio transmission. A message is sent from a source to the recipient who does not know the true message and furthermore experiences some interference. The recipient must recover the message based on its relationship to some known "predictor", by separating the true message form the random noise. This broad topic is divided into parametric regression and nonparametric regression. As we shall see, the use of each method depends on the amount of information that is available about the regression function before it is estimated. The earliest description of regression appears in the work of Francis Galton (1886) who coined the term and applied simple linear regression to the problem of predicting hereditary traits. One of the first applications of nonparametric regression dates to 1857 when the economist Ernst Engel analyzed Belgian data on household expenditure by using a method now known as a regressogram.

At the time of undertaking this project, we were interested in a variety of topics in nonparametric regression theory. Our main goal was to study various aspects of nonparametric regression analysis in enough detail to allow us to apply nonparametric regression techniques in practice.

Some well-known introductory texts on the subject, such as Härdle et al. (2000) and Wasserman (2006) merely describe some methods of nonparametric regression with very little theoretical justification. Indeed, many textbooks on the subject do not give a thorough treatment of the theory, especially concerning methods of smooth- 
ing parameter selection. On the other hand, a wealth of articles and texts exist that prove important results for certain nonparametric regression topics, but that are too technical and either sketch or altogether omit the details of some important proofs. Texts such as Eubank (1999) and Härdle (1989) offer a compromise, but cover very specific topics with varying degrees of detail. Our goal is to discuss three important techniques of nonparametric regression in detail, namely kernel regression estimation, orthogonal series estimation, and spline smoothing. We justify each method theoretically and apply it to a simulated data set. For this purpose, S-Plus functions are written for kernel regression and orthogonal projection estimation including the corresponding parameter selection techniques. While, spline smoothing and corresponding parameter selection procedures are obtained by using the smooth.spline() function already available from S-Plus.

\subsection{Fixed Design Regression Model}

The fixed design regression model assumes that observations $Y_{1}, \ldots, Y_{n}$ satisfy

$$
Y_{i}=f\left(x_{i}\right)+\varepsilon_{i}, \quad i=1, \ldots, n
$$

where $f$ is an unknown regression function, $x_{i}$ are design points fixed by the experimenter, and $\varepsilon_{i}$ are independent $N\left(0, \sigma^{2}\right)$ normal random errors. We shall discuss two popular designs $\left\{x_{i}\right\}_{i=1}^{n}$, uniform design and Chebyshev design. Now, put

$$
\begin{gathered}
\mathbf{Y}=\left(Y_{1}, \ldots, Y_{n}\right)^{\top} \\
\mathbf{f}=\left(f\left(x_{1}\right), \ldots, f\left(x_{n}\right)\right)^{\top} \\
\boldsymbol{\varepsilon}=\left(\varepsilon_{1}, \ldots, \varepsilon_{n}\right)^{\top} .
\end{gathered}
$$


Then, in matrix notation, model (1) has the form

$$
\mathbf{Y}=\mathbf{f}+\boldsymbol{\varepsilon}
$$

Under model (1), or (2), the regression function $f$ is unknown and must be estimated given a realization $\mathbf{y}=\left(y_{1}, \ldots, y_{n}\right)^{\top}$ of $\mathbf{Y}$. This can be done by using parametric regression or nonparametric regression depending on how much we know about the form of $f$.

\subsection{Nonparametric Regression and Review of Literature}

Parametric regression requires the knowledge of the form of $f$ up to a finite number of unknown parameters that are then estimated from the data. Typically, the parametric regression estimator of $f$ converges to the true regression function in mean square error at the rate of $n^{-1 / 2}$. However, the form of the true regression function is not usually known in practice, so parametric regression is not always appropriate. If one has little knowledge about the form of the true regression function, an arbitrary selection of the form may result in an estimator that does not converge to the true regression function or one that has a slower rate of convergence. For a practical example, see Gasser et al. (1984), who showed this drawback of parametric regression in the context of human growth data. In this example, a misspecified form of the regression function leads to incorrect inferences. In such cases it is preferable to use only the information contained in the data.

Nonparametric regression makes no assumptions about the form of the regression function $f$ other than that it belong to some infinite-dimensional collection of functions $\mathcal{F}=\{f\}$. Therefore, one only chooses an appropriate functional space $\mathcal{F}$ that is believed to contain the regression function, and uses the data to estimate the 
regression function from among that class. Nonparametric regression estimators are widely studied in statistical literature, since they are mathematically attractive and have many useful applications.

In the early 1980s, an approach with emphasis on optimal minimax rates of convergence for estimating an unknown regression function from a certain functional class became very popular. See Golubev, Levit, and Tsybakov (1996) for a good overview. Consider the problem of estimating the regression function $f$ in model (1)

$$
Y_{i}=f\left(x_{i}\right)+\varepsilon_{i}, \quad i=1, \ldots, n,
$$

where $x_{i}$ are fixed design points belonging to some bounded or unbounded observation interval, and where $f$ is an unknown regression function that belongs to some functional class $\mathcal{F}=\{f\}$. For any $x$ from the observation interval, denote by $f_{n}(x)=f_{n}\left(x, Y_{1}, \ldots, Y_{n}\right)$ an estimator of $f(x)$ based on the observations $Y_{1}, \ldots, Y_{n}$. A reasonable estimator $f_{n}$ should be such that $f_{n}-f \rightarrow 0$ as $n \rightarrow \infty$. Therefore, we have to norm the difference, $f_{n}-f$, through multiplication by a factor $r_{n}^{-1}$, where $r_{n} \rightarrow 0$ as $n \rightarrow \infty$. For fixed $x$, an estimator $f_{n}^{*}(x)$ is called asymptotically minimax, or efficient estimator of $f(x)$ if it asymptotically minimizes the maximum risk:

$$
\lim _{n \rightarrow \infty} \sup _{f} \mathbf{E}_{f}\left(r_{n}^{-1}\left(f_{n}^{*}(x)-f(x)\right)\right)^{2}=\lim _{n \rightarrow \infty} \inf _{f_{n}} \sup _{f} \mathbf{E}_{f}\left(r_{n}^{-1}\left(f_{n}(x)-f(x)\right)\right)^{2},
$$

where the infimum is taken over all estimators $f_{n}$, and the supremum over all $f \in \mathcal{F}$, and $r_{n}$ is the minimax rate of convergence. That is, $r_{n}$ is such that

$$
\lim _{n \rightarrow \infty} \sup _{f \in \mathcal{F}} \mathbf{E}_{f}\left(r_{n}^{-1}\left(f_{n}^{*}(x)-f(x)\right)\right)^{2}=c^{2}(x)
$$

where $c(x)$ is a positive bounded quantity that may depend on $x$, called the exact 
asymptotic constant. The faster the rate of convergence (the growth of the factor $r_{n}^{-1}$ as $n \rightarrow \infty)$ is for an estimator, the better the estimator. Exact estimation of $f$ refers to the problem of finding both the optimal rate of convergence and the exact asymptotic constant, and of deriving the corresponding asymptotically minimax estimator. In some interesting cases, the problem of exact estimation was addressed in the work of Pinsker (1980), Nussbaum (1985), Golubev and Levit (1996), Guerre and Tsybakov (1998), Levit and Stepanova (2004).

When dealing with the problem of exact estimation of $f$, one distinguishes between two cases: (i) an unknown regression function $f$ is observed on a grid $\left\{x_{i}\right\}$ on the entire real line, and (ii) an unknown regression function $f$ is observed on a grid $\left\{x_{i}\right\}$ on a bounded interval $[a, b]$ of the real line. In practice, observations of the regression function $f(x)$ are available on a bounded interval. For this reason, we consider the bounded interval case in this thesis as it is more realistic. Compared to regression on the real line, regression on a bounded interval suffers from the so-called boundary effect when the accuracy of estimation at the end-points of the observation interval is worse compared to the accuracy obtained inside the interval. In other words, the asymptotic variance of an estimator is greater at the end-points (the value of $c(x)$ at $x=a$ and $x=b$ is greater compared to its value at $x \in(a, b))$. This effect was studied in detail in Artiles (2001), Section 3. The boundary effect will be illustrated in Chapter 2 in connection with estimating $f$ by means of orthogonal series estimators. Loss of accuracy of estimation near the boundary is not a drawback of any specific method of estimation, but rather a built-in defect of the design, especially of uniform design. For this reason, the presence of the boundary must be incorporated into the study of optimal statistical procedures. For example, the situation can be improved by using a non-uniform design, which balances the distribution of the design points in favour of increasing the accuracy of estimation at the end-points. A classical 
design with this property is the Chebyshev design. For the remainder of this thesis, the observation interval $[a, b]$ is assumed to be bounded, and the design points are assumed to be arranged from the smallest to the largest: $a \leq x_{1}<\ldots<x_{n} \leq b$.

For most parametric estimators, when the underlying distribution is assumed to be known up to a finite number of unknown parameters, the risk decays to zero at a rate of $n^{-1 / 2}$. The corresponding rate for nonparametric estimators is lower: usually $n^{-(1-\delta) / 2}$, where $\delta \in(0,1)$. The exact value of $\delta$ depends on the smoothness of $f$. In order to find the best possible rate of convergence for estimating a function $f$ most accurately, one must assume that $f$ comes from a specific family of classes $\left\{\mathcal{F}_{\beta}\right\}$. These can be divided into two categories: classes of finite smoothness and infinite smoothness. Much work has been done on the relationship between the smoothness of a regression function $f$ and the optimal rate of convergence. Typically a smoother estimable function $f$ leads to a faster rate of convergence of the minimax estimator $f_{n}^{*}(x)$.

During the 1980s and 1990s, functional estimation was dominated by the assumption that the unknown function $f$ belongs to some functional class of finite smoothness. One well-known example is the collection of Hölder classes $\left\{\mathcal{F}_{\beta}(M)\right\}$ with $\beta=k+\alpha, 0 \leq \alpha \leq 1$, and $k \geq 1$. These classes contain functions defined on the real line that are smooth to the order $k$ and are such that $f^{(k)}$ satisfies the Hölder condition with exponent $\alpha$ and constant $M>0$. It is well-known that the optimal rate of convergence in estimating $f(x)$ when $f \in \mathcal{F}_{\beta}(M)$ is $r_{n}=n^{-\beta /(2 \beta+1)}$, see, for example, Prokhorov (1999), p. 395. Another example of a collection of finite smoothness classes is the set $\mathcal{F}_{m}(M)$ of all functions on $[0,1]$ that, for some integer $m \geq 1$ and a finite positive constant $M$, have $m-1$ absolutely continuous derivatives and satisfy $\int_{a}^{b}\left(f^{(m)}(x)\right)^{2} d x \leq M$. Speckman (1985) established that, in the case of uniform design, the optimal rate of convergence of a linear regression estimator $f_{n}$ to 
$f \in \mathcal{F}_{m}(M)$ using the risk $R\left(f_{n}, f\right)=n^{-1} \sum_{i=1}^{n} \mathbf{E}\left(f_{n}\left(x_{i}\right)-f\left(x_{i}\right)\right)^{2}$ is $r_{n}=n^{-m /(2 m+1)}$. Moreover, Golubev and Nussbaum (1990) presented results showing that this rate is optimal among all estimators. Efromovich (1996) generalized this result for the case of random design. In this thesis, we discuss several functional spaces of finite smoothness that are subclasses of $L_{2}[a, b],-\infty<a<b<\infty$, the collection of all square integrable functions on the interval $[a, b]$.

Classes of analytic (infinitely-smooth) functions can be studied in the same setting, for a large variety of estimation schemes. Minimax estimators for infinitelydifferentiable functions were first constructed by Ibragimov and Hasminskii (1983, 1984). Functions $f \in \mathcal{F}_{\gamma}(M)$ that are analytic and bounded in absolute value by $M$ on a symmetric strip of size $2 \gamma$ around the real axis have been considered for various statistical models. See, for example, Parzen (1958), Ibragimov and Hasminskii (1983), Golubev and Levit (1996), Golubev, Levit, and Tsybakov (1996), Guerre and Tsybakov (1998), Levit and Stepanova (2003). Unlike the more familiar finite smoothness classes, they yield nonparametric estimators of $f(x)$ for which, not only the optimal rate of convergence, but also the exact minimax constants are known. The corresponding rate of convergence is $r_{n}=\left(n^{-1} \ln n\right)^{1 / 2}$, which is better than the rates for finite smoothness classes.

We have just seen that the optimal estimator $f_{n}^{*}$ depends on the choice of $\mathcal{F}$. However, choosing a suitable functional class $\mathcal{F}=\{f\}$ does not mean finding the "true" functional class of $f$. Indeed, we will probably never know the "true" smoothness of an estimable function $f$. For this reason, adaptive estimation is a widely used alternative to minimax estimation. Adaptive estimation addresses this problem by assuning that an unknown function $f$ may be, in reality, of any smoothness. By adaptive methods of estimation we refer to data-driven methods that, in a sense, adapt to the uncertainty about the actual class $\mathcal{F}$. The construction of optimal adaptive procedures for 
estimating a regression function is a complex problem and is not addressed in this thesis. In practice, when dealing with problems of nonparametric regression analysis, cross-validation and generalized cross-validation methods are often used. These two adaptive methods perform reasonably well compared to efficient estimation procedures that require knowledge of the smoothness of $f \in \mathcal{F}$. More details are given in Chapter 5.

\subsection{Measures of Fit}

The problem of selecting a nonparametric regression estimator consists of choosing, out of a class of estimators, the best estimator in terms of some predetermined criterion, called a measure of fit or a performance criterion. A performance criterion is a measure of proximity between the true regression function $f(x)$ and an estimator $f_{n}(x)=f_{n}(x, \mathbf{Y})$. There are several commonly used measures of fit. Typically, in order to calculate their values one must know the true regression function. In practice a criterion must be estimated from the data, and minimized with respect to the function $f_{n}$ chosen from a certain class of estimators.

The proximity between $f_{n}$ and $f$ is assessed by using a loss function. One popular choice of loss function is the (normalized) Euclidian distance between the vector of values of regression function $\mathbf{f}=\left(f\left(x_{1}\right), \ldots, f\left(x_{n}\right)\right)^{\top}$ and the vector of

regression estimates at the design points $\mathbf{f}_{n}=\left(f_{n}\left(x_{1}\right), \ldots, f_{n}\left(x_{n}\right)\right)^{\top}$. This loss is sometimes known as the average squared error (ASE):

$$
L\left(f_{n}, f\right)=\frac{1}{n} \sum_{i=1}^{n}\left(f\left(x_{i}\right)-f_{n}\left(x_{i}\right)\right)^{2}
$$

The expected value of the loss function is called the risk, also known as the mean 
average squared error (MASE):

$$
R\left(f_{n}, f\right)=\mathbf{E}\left(\frac{1}{n} \sum_{i=1}^{n}\left(f\left(x_{i}\right)-f_{n}\left(x_{i}\right)\right)^{2}\right)
$$

The quantities $L\left(f_{n}, f\right)$ and $R\left(f_{n}, f\right)$ are local measures of fit, because they provide a performance measure of the regression estimator at the design points. These can be modified to become global measures of fit by integrating them over the observation interval $[a, b]$. The integrated loss, also known as integrated squared error (ISE), is defined by

$$
I L\left(f_{n}, f\right)=\int_{a}^{b}\left(f(x)-f_{n}(x)\right)^{2} d x
$$

Then the integrated risk, also known as mean integrated squared error (MISE), is given by

$$
I R\left(f_{n}, f\right)=\mathbf{E}\left(\int_{b}^{a}\left(f(x)-f_{n}(x)\right)^{2} d x\right)
$$

Another local performance criterion is the prediction risk or mean squared error (MSE). It is used to measure the performance of the estimator $\mathbf{f}_{n}=\left(f_{n}\left(x_{1}\right), \ldots, f_{n}\left(x_{n}\right)\right)^{\top}$ obtained from (2) for predicting $n$ new observations from $\mathbf{Y}^{*}=\mathbf{f}+\boldsymbol{\varepsilon}^{*}$, where $\mathbf{f}$ is defined as above and $\varepsilon^{*}$ is a vector of independent $N\left(0, \sigma^{2}\right)$ random variables indepdentent of $\varepsilon$. The prediction risk is defined by

$$
P\left(f_{n}, f\right)=\frac{1}{n} \sum_{i=1}^{n} \mathbf{E}\left(Y_{i}^{*}-f_{n}\left(x_{i}\right)\right)^{2}
$$

There is no one generally accepted measure of performance of a regression estimator. When estimating a regression function in the nonparametric setting, one must choose 
a performance criterion first, and then find the estimator that minimizes this criterion among some class of estimators that is believed to contain the true regression function.

The performance criteria described here are all related to one another. Indeed, using the independence of $\varepsilon$ and $\varepsilon^{*}$ and the fact that $\mathrm{E} \varepsilon_{i}^{*}=0$, the prediction risk can be expressed as a function of the risk as follows:

$$
\begin{aligned}
P\left(f_{n}, f\right) & =\frac{1}{n} \sum_{i=1}^{n} \mathbf{E}\left(Y_{i}^{*}-f_{n}\left(x_{i}\right)\right)^{2}=\frac{1}{n} \sum_{i=1}^{n} \mathbf{E}\left(f\left(x_{i}\right)+\varepsilon_{i}^{*}-f_{n}\left(x_{i}\right)\right)^{2} \\
& =\frac{1}{n} \sum_{i=1}^{n} \mathbf{E}\left(f\left(x_{i}\right)-f_{n}\left(x_{i}\right)\right)^{2}+\frac{1}{n} \sum_{i=1}^{n} \mathbf{E}\left(\varepsilon_{i}^{*}\right)^{2}+\frac{2}{n} \sum_{i=1}^{n} \mathbf{E}\left(\varepsilon_{i}^{*}\left(f\left(x_{i}\right)\right)-f_{n}\left(x_{i}\right)\right) \\
& =R\left(f_{n}, f\right)+\sigma^{2} .
\end{aligned}
$$

Thus, minimizing one of these criteria also minimizes the others. In other words, the choice of the regression estimator is not affected by the choice of a performance criterion from the above.

\subsection{Linear Estimators}

An estimator of $f$ must use the information about the response available at the design points $x_{i}$ to estimate values of the regression function at non-design points. As before, consider the fixed design model (1) or (2). A linear estimator of $f$ "weighs" the contribution of each of the $n$ response observations towards the estimation of the regression function at a point $x$, not necessarily a design point.

Definition 1. A linear estimator is an estimator that can be written in the form

$$
f_{n}(x)=\sum_{i=1}^{n} k_{n}\left(x, x_{i} ; h\right) Y_{i},
$$

where $k_{n}(x, y ; h)$ is a weight function independent of the design. It weighs the 
$i$ th response according to some scaled (by means of the smoothing parameter $h=$ $h_{n}$ ) measure of distance between $x_{i}$ and the point of evaluation $x$. Putting $\mathbf{f}_{n}=$ $\left(f_{n}\left(x_{1}\right), \ldots, f_{n}\left(x_{n}\right)\right)^{\top}$ and defining an $n \times n$ non-random matrix

$$
\mathbf{S}_{n}=\left(\mathbf{S}_{i j}\right)_{i, j=1}^{n}, \quad \mathbf{S}_{i j}=\mathbf{S}_{i j}^{(n)}=k_{n}\left(x_{i}, x_{j} ; h\right)
$$

yields the vector of estimates $f_{n}\left(x_{i}\right)$ in the form

$$
\mathbf{f}_{n}=\mathbf{S}_{n} \mathbf{Y}
$$

Although it is possible to create an estimator with asymmetric weights, the regression estimators discussed in this thesis are linear in the design and have symmetric weights, i.e. $k_{n}(x, y ; h)=k_{n}(y, x ; h)$, which implies symmetry of the $\mathbf{S}_{n}$ matrix. Therefore, for the remainder of this thesis, we will deal with a symmetric $\mathbf{S}_{n}$ matrix, unless otherwise specified. 


\section{Kernel Regression Estimation}

A kernel estimator of the unknown regression function $f$ from model (1) is a linear estimator obtained by weighting the response values $Y_{i}, i=1, \ldots, n$, by a so-called kernel function or simply kernel. Kernel estimators were first studied in relation to density estimation by Rosenblatt (1956) and Parzen (1962). Priestly and Chao (1972) generalized the Parzen-Rosenblatt kernel estimator by adding flexibility under a nonuniform design. Subsequently, various kernel regression estimators were suggested by Nadaraya (1964) and Watson (1964), Gasser and Muller (1979) and others. This chapter gives an intuitive justification for using a Parzen-Rosenblatt kernel estimator for regression function estimation and informally presents the derivation of its asymptotic risk. Several types of kernel estimators are discussed and their asymptotic risk is given. The chapter ends with a short note that, under some conditions on the kernel, the Parzen-Rosenblatt kernel estimator is closely related to other nonparametric estimators.

Return to model (1) and consider a linear estimator $f_{n}$ of $f$ :

$$
f_{n}(x)=\sum_{i=1}^{n} k_{n}\left(x, x_{i} ; h\right) Y_{i}
$$

The weights for kernel estimators are obtained by an intuitive argument and are then shown to have desirable properties of approximation. More precisely, a kernel estimator with kernel $K$ and bandwidth $h$ is defined as

$$
f_{n}(x)=\frac{1}{n} \sum_{i=1}^{n} \frac{1}{h} K\left(\frac{x-x_{i}}{h}\right) Y_{i}
$$

where $h=h_{n} \rightarrow 0$ and $n h \rightarrow \infty$, as $n \rightarrow \infty$, and $K$ satisfies the following properties. 
First, it integrates to one:

$$
\int_{-\infty}^{\infty} K(u) d u=1
$$

Second, $K$ is assumed symmetric about 0 , which is ensured the by condition

$$
\int_{-\infty}^{\infty} u K(u) d u=0
$$

and attains a maximum at zero:

$$
\max _{x} K(x)=K(0)
$$

Also, as will be seen later, the following two assumptions lead to some desirable convergence properties of the kernel regression estimator:

$$
\begin{gathered}
\int_{-\infty}^{\infty} u^{2} K(u) d u \neq 0 \\
\int_{-\infty}^{\infty} K^{2}(u) d u<\infty
\end{gathered}
$$

Note that we do not restrict a kernel function to be non-negative. Moreover, we allow kernel functions $K_{n}(x)$ that depend on $n$. It is generally accepted that the choice of the kernel $K$, or $K_{n}$, is far less crucial for the quality of $f_{n}$ as an estimator of $f$ than the choice of the bandwidth $h=h_{n}$. However, as shown, for example, in Guerre and Tsybakov (1998), this is not always true. In the more delicate problems of exact efficient and exact adaptive estimation, when not only the rate of convergence but also the exact asymptotic constant is of interest, the choice of a suitable kernel is as 
crucial as the choice of bandwidth. Table 2 gives some non-standard kernel functions that first appeared in the literature in the context of exact efficient estimation of density functions and of regression functions.

\section{$2.1 \quad$ Kernel Functions}

We have defined a kernel function, or kernel, as any function that has properties (3) - (7). Some commonly used kernel functions are given in Table 1. These standard kernel functions have the additional property that $K(x) \geq 0, x \in \mathbb{R}$. The uniform and Epanechnikov kernels have finite support, while the Gaussian kernel has infinite support.

\begin{tabular}{lll}
\hline Kernel & $K(x)$ & $\widehat{K}(t)$ \\
\hline \hline Uniform & $\frac{1}{2} \mathbb{I}(|x| \leq 1)$ & $\frac{\sin t}{t}$ \\
Epanechnikov & $\frac{3}{4}\left(1-x^{2}\right) \mathbb{I}(|x| \leq 1)$ & $\frac{3 \sin t}{t^{3}}-\frac{3 \cos t}{t^{2}}$ \\
Gaussian & $\frac{1}{\sqrt{2 \pi}} \exp \left(-\frac{x^{2}}{2}\right)$ & $\exp \left(-\frac{t^{2}}{2}\right)$ \\
\hline
\end{tabular}

Table 1: Some standard kernel functions and their Fourier transforms.

The kernel functions in Table 2 are less known. They first arose in problems of exact efficient estimation as leading to the best achievable rates of convergence and to the smallest exact asymptotic constants. 


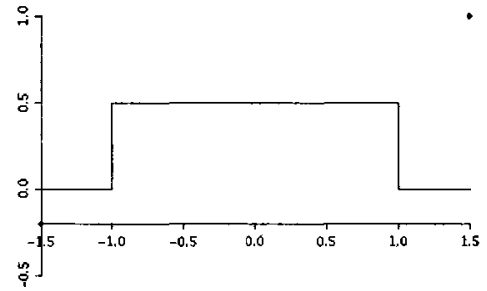

(a) Uniform kernel function

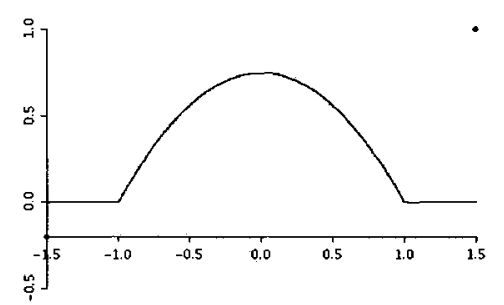

(b) Epanechnikov kernel function

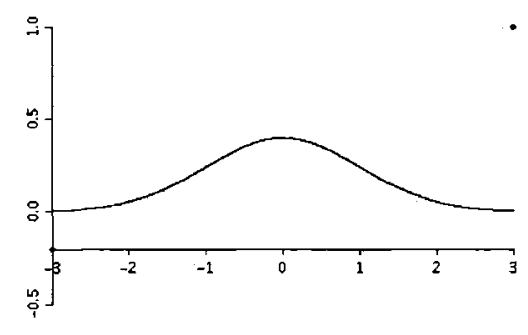

(c) Gaussian kernel function

Figure 1: Some well-known kernels.

\begin{tabular}{|c|c|c|}
\hline Kernel & $K(x)$ or $K_{n}(x)$ & $\widehat{K}(t)$ or $\widehat{K}_{n}(t)$ \\
\hline de la Vallée Poussin & $\frac{\cos (x)-\cos (2 x)}{\pi x^{2}}$ & 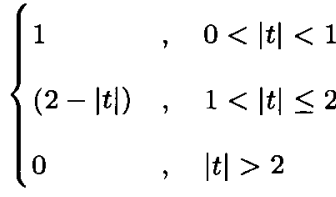 \\
\hline Golubev-Levit & $\sqrt{\frac{n}{n-1}} \frac{\sin \left(x \cosh ^{-1}(2 n-1)\right)}{\sinh (\pi x)}$ & $\frac{n}{n+\sinh ^{2}(t / 2)}$ \\
\hline Fejér & $\frac{\sin x}{\pi x}$ & $\begin{cases}1, & |t|<1 \\
0, & |t|>1\end{cases}$ \\
\hline
\end{tabular}

Table 2: Some non-standard kernel functions and their Fourier transforms.

These kernels alternate between positive and negative values. All three kernels satisfy properties (3)-(7). The Fejér kernel is used by several authors. Notably, Guerre and Tsybakov (1998) use it to construct an efficient kernel estimator of an unknown 
infinitely-smooth signal in the continuous analog of model (1). A drawback of the Fejér kernel is that it is not absolutely integrable. This fact greatly complicates the theoretical study of the properties of the corresponding estimator. The Golubev-Levit kernel first appeared in Golubev and Levit (1996) in connection with the estimation of the infinitely-smooth distribution function $F$ and its derivatives $F^{(m)}, m \geq 1$. The kernel-type estimator of $F^{(m)}, m \geq 0$, based on this kernel was shown to be asymptotically efficient. The de la Vallée Poussin kernel was borrowed from Approximation Theory (see Nikolskii, 1975), and successfully used in the field of functional estimation by Ibragimov and Hasminskii $(1983,1984)$. Any of these three non-standard kernels are preferred to any of the standard kernels, because the exact asymptotic risk of the corresponding kernel estimators is smaller than the risk of estimators based on standard kernels.

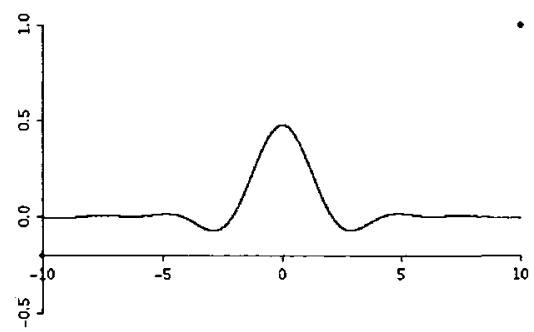

(a) De La Vallée Poussin kernel

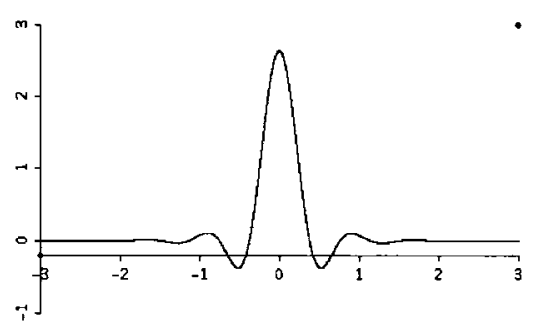

(b) Golubev-Levit kernel

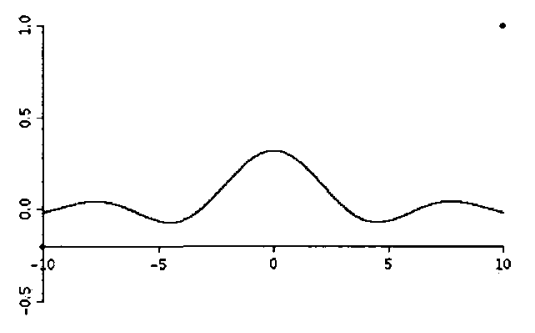

(c) Fejér kernel

Figure 2: Some uncommon kernel functions 


\section{$2.2 \quad$ Kernel Regression Estimators}

Let us return to the problem of estimating the regression function $f$ in the fixed design model (1)

$$
Y_{i}=f\left(x_{i}\right)+\varepsilon_{i}, \quad i=1, \ldots, n,
$$

where $x_{i}$ are fixed design points, or knots, inside the observation interval $[a, b]=[0,1]$ and where $\varepsilon_{i}$ are independent $N\left(0, \sigma^{2}\right)$ normal random errors. We now discuss finding a set of intuitive weight functions for the linear regression estimator

$$
f_{n}(x)=\sum_{i=1}^{n} k_{n}\left(x, x_{i} ; h\right) Y_{i} .
$$

We want the kernel weight associated with the $i$ th design point to be a function of the scaled distance between the point of evaluation $x$ and the design point $x_{i}$. For this, define the scaled kernel

$$
K_{h}(x)=\frac{1}{h} K\left(\frac{x}{h}\right)
$$

where the smoothing parameter $h=h_{n}$ is chosen by the experimenter in such a way that

$$
h \rightarrow 0 \quad \text { and } \quad n h \rightarrow \infty, \quad \text { as } n \rightarrow \infty .
$$

Then the regression function $f$ at a point $x$ can be approximated by a weighted average of the $n$ responses $Y_{1}, \ldots, Y_{n}$, with weights given by the scaled kernel (8). This weighted average, called a kernel regression estimator or simply kernel 
estimator, has the form

$$
f_{n}(x)=\frac{1}{n} \sum_{i=1}^{n} K_{h}\left(x-x_{i}\right) Y_{i}
$$

As will be shown later, the above assumption on $h$ implies that $f_{n}(x)$ is a consistent estimator of $f(x)$. Originally, the kernel estimator was introduced by Rosenblatt (1956) and Parzen (1962) for estimating an unknown density function. It was subsequently adjusted to the purpose of regression estimation. Estimator (9) gives each response value $Y_{i}$ a weight proportional to the distance between $x$ and $x_{i}$ scaled by $h$. The smaller the distance between the design point $x_{i}$ and $x$, the more weight is given to the corresponding response variable $Y_{i}$. The smoothing parameter $h$, which is simply a compression factor of the kernel, governs the effect that the design will have on the weighting of each observation. As a result the kernel estimator's smoothness is directly proportional to the value of $h$. Some methods for selecting the smoothing parameter are discussed in Chapter 5.

In Figures 3 and 4, a data set with 40 observations was generated from model (1) under the design $x_{i}=\frac{2 i}{2 n+1}, i=-n, \ldots,-1,0,1, \ldots, n$ with $\varepsilon_{i}$ being independent $N\left(0,(0.2)^{2}\right)$ normal random variables. As expected from theory, the nonstandard kernels (De La Vallée Poussin, Golubev-Levit and Fejér) seem to provide estimates that are closer to the true regression function than the estimates generated by using the standard kernels. 


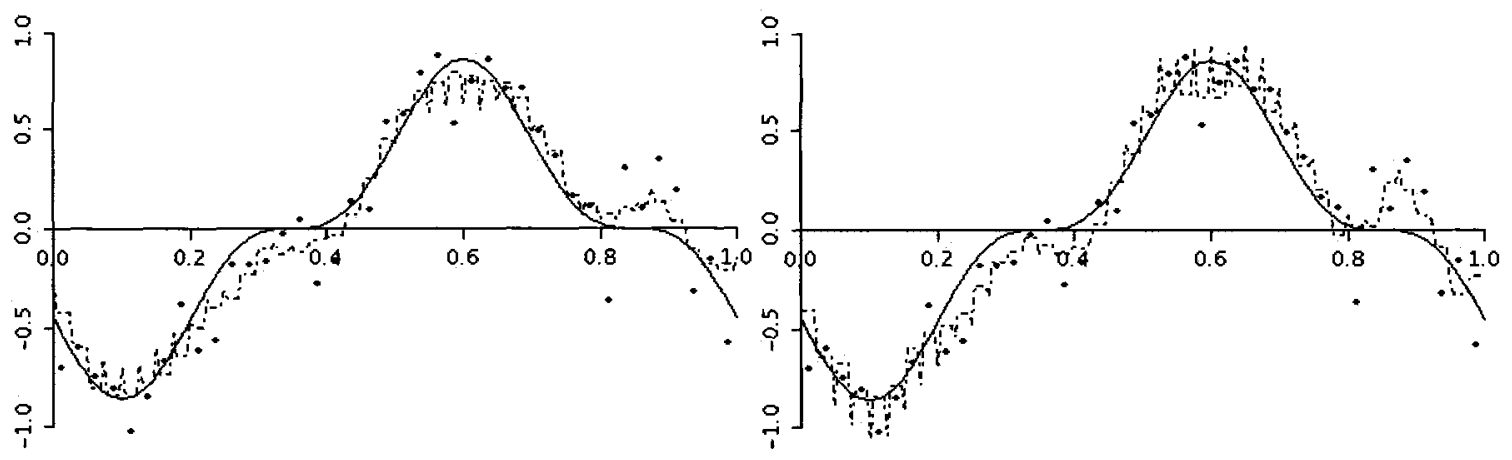

(a) Uniform kernel, $h_{C V}=0.06$

(b) Uniform kernel, $h_{G C V}=0.04$

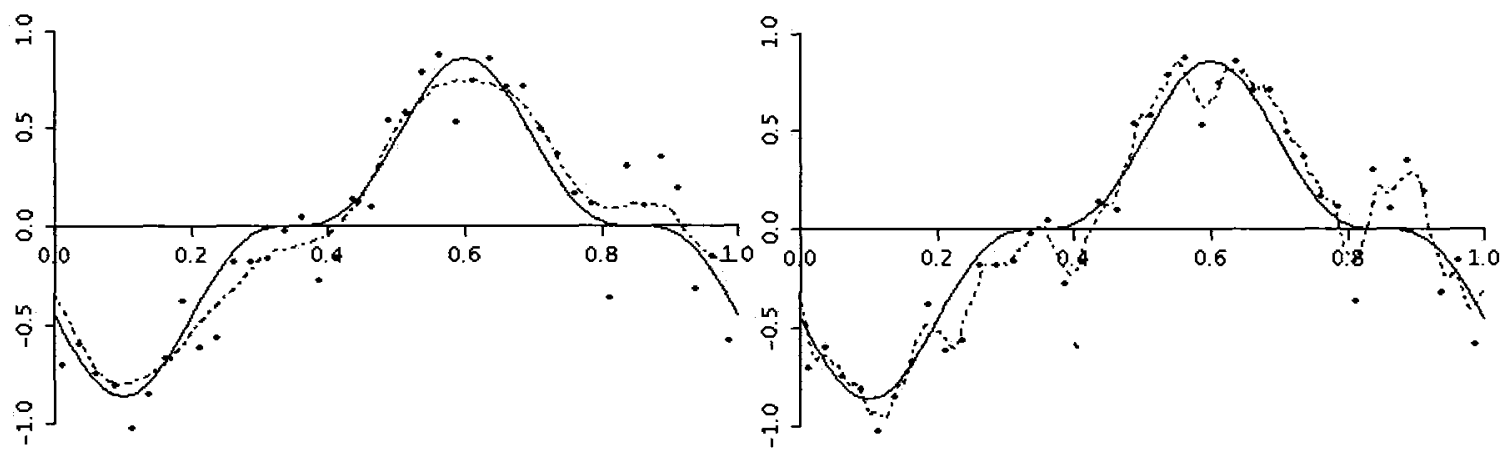

(c) Epanechnikov kernel, $h_{C V}=0.09$

(d) Epanechnikov kernel, $h_{G C V}=0.003$

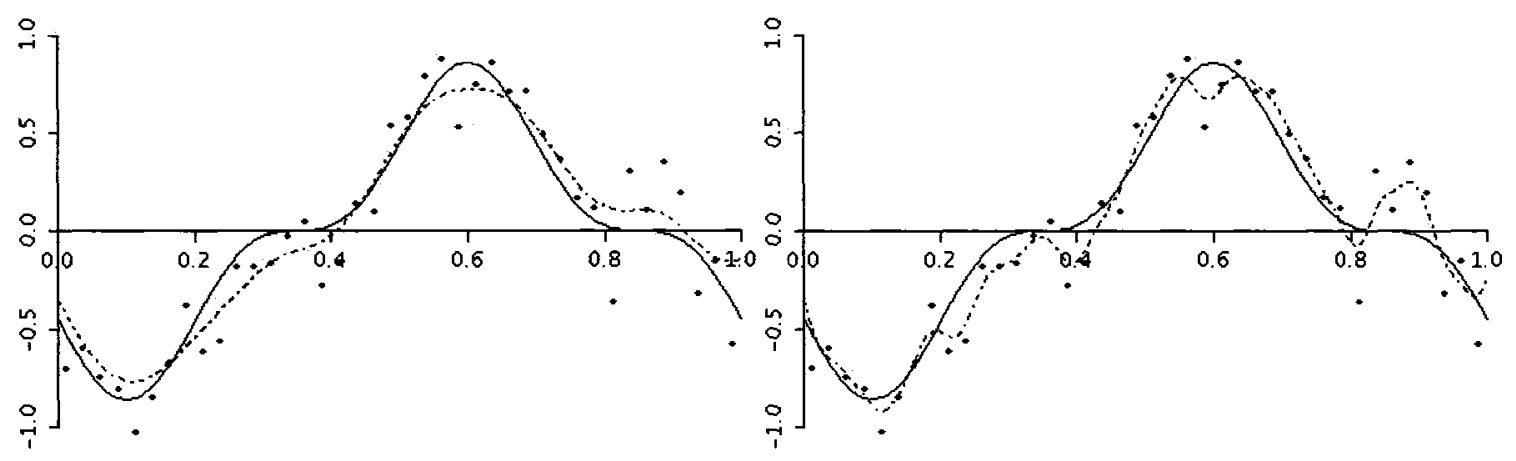

$\begin{array}{ll}\text { (e) Gaussian kernel, } h_{C V}=0.05 & \text { (f) Gaussian kernel, } h_{G C V}=0.02\end{array}$

Figure 3: The kernel regression estimator $f_{2 n+1}(x)=\frac{1}{2 n+1} \sum_{i=-n}^{n} K_{h}\left(x-x_{i}\right) Y_{i}$ is shown (dotted line), where $K_{h}(\cdot)$ is a standard kernel. The estimator is based on $2 n+1=40$ data points from $Y_{i}=f\left(x_{i}\right)+\varepsilon_{i}$, where $x_{i}=\frac{2 i}{2 n+1}, i=-n, \ldots,-1,0,1, \ldots, n$. The regression function is given by $f(x)=-0.95 \cos ^{3}(2 \pi x+1.6 \pi)$ (solid line) and the error terms $\varepsilon_{1}, \ldots, \varepsilon_{n}$ are randomly generated from a normal distribution with mean 0 and standard deviation 0.2. The smoothing parameters $h_{C V}$ and $h_{G C V}$ were obtained by the methods of cross-validation, and generalized cross-validation respectively (see Chapter 5 for details). 


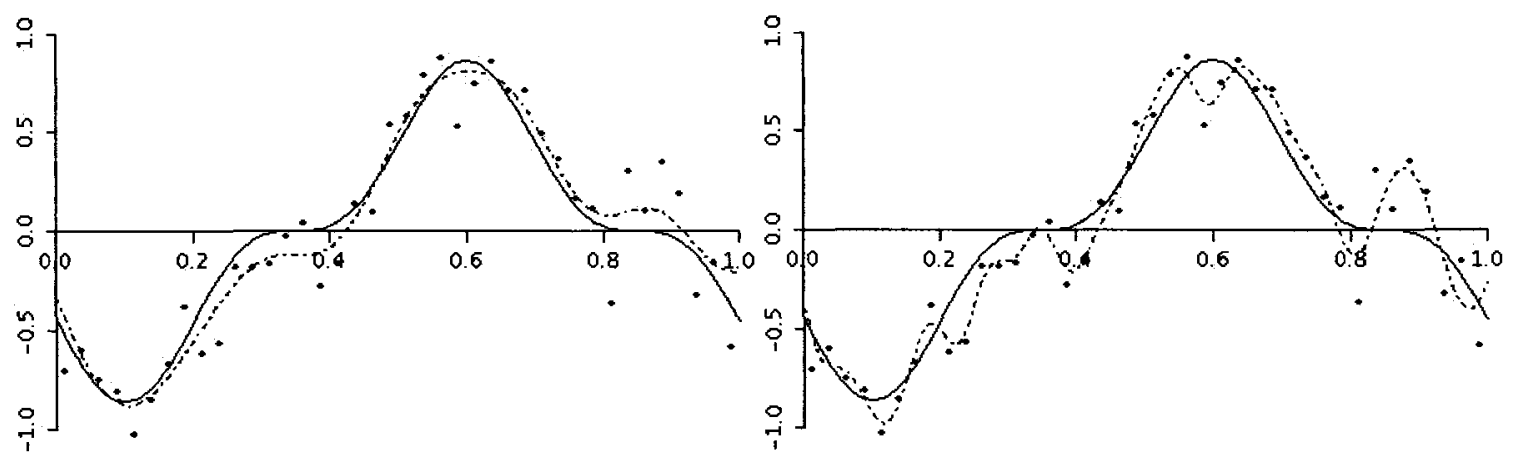

(a) De La Vallée Poussin kernel, $h_{C V}=0.005$

(b) De La Vallée Poussin kernel, $h_{G C V}=0.002$

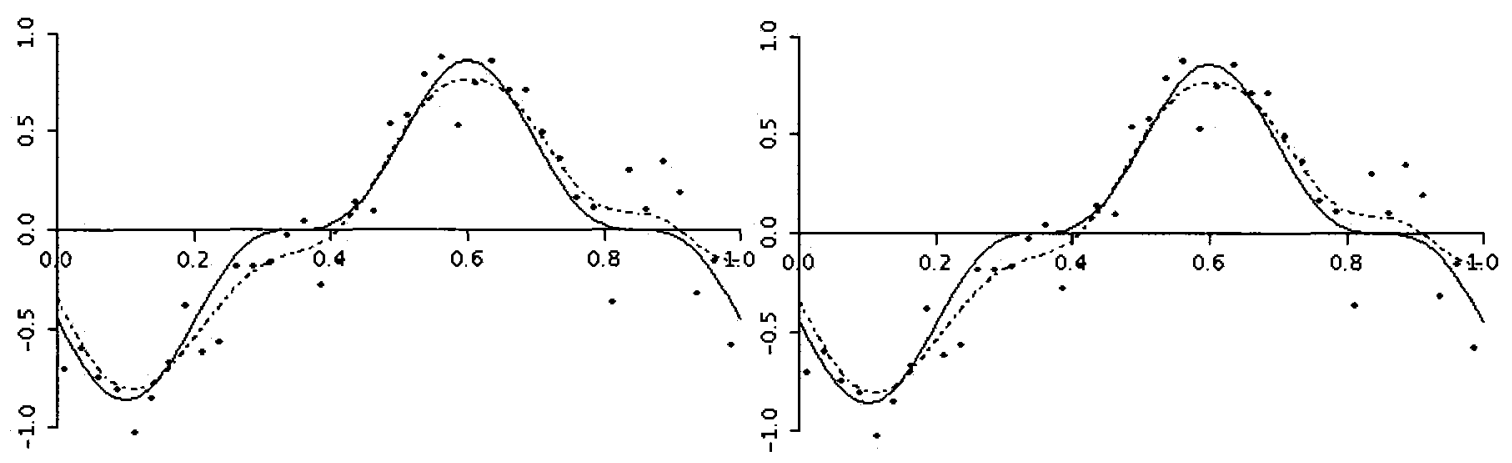

(c) Golubev-Levit kernel, $h_{C V}=0.2$

(d) Golubev-Levit kernel, $h_{G C V}=0.15$

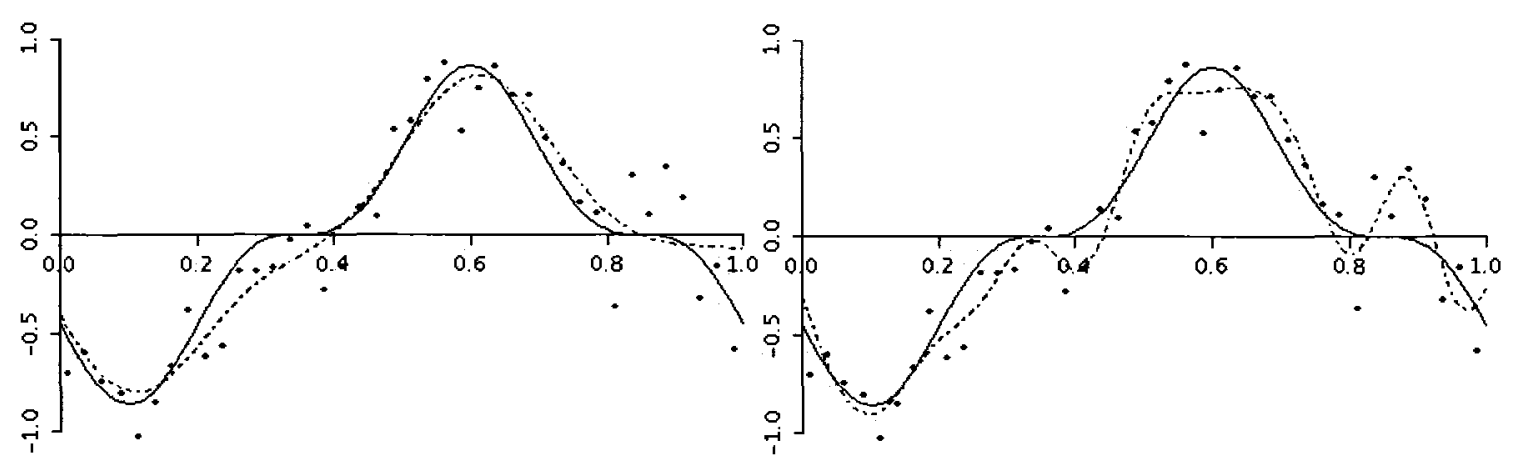

$\begin{array}{ll}\text { (e) Fejér kernel, } h_{C V}=0.05 & \text { (f) Fejér kernel, } h_{G C V}=0.02\end{array}$

Figure 4: The kernel regression estimator $f_{2 n+1}(x)=\frac{1}{2 n+1} \sum_{i=1}^{n} K_{h}\left(x-x_{i}\right) Y_{i}$ is shown (dotted line), where $K_{h}(\cdot)$ is a non-standard kernel. The estimator is based on $2 n+1=40$ data points from $Y_{i}=f\left(x_{i}\right)+\varepsilon_{i}$, where $x_{i}=\frac{2 i}{2 n+1}, i=-n, \ldots,-1,0,1, \ldots, n$. The regression function is given by $f(x)=-0.95 \cos ^{3}(2 \pi x+1.6 \pi)$ (solid line) and the error terms $\varepsilon_{1}, \ldots, \varepsilon_{n}$ are randomly generated from a normal distribution with mean 0 and standard deviation 0.2. The smoothing parameters $h_{C V}$ and $h_{G C V}$ were obtained by the methods of cross-validation, and generalized cross-validation respectively (see Chapter 5 for details). 
Another interesting observation from Figures 3 and 4 is that the bandwidths selected by the method of cross-validation provide a much better fit to the data than those provided by generalized cross-validation. As we will see in Chapter 5, this is due to the fact that while cross-validation is an intuitively reasonable approach to selecting the bandwidth, generalized cross-validation is simply a modification of the regression sum of squares that approximates the cross-validation score for large $n$. For small sample sizes, generalized cross-validation does not differ much from the regression sum of squares, which tends to emphasize under-smoothing, and therefore leads to lower estimates of the optimal bandwidth. Our simulated data set is of a "moderate" size, and thus we expect to obtain under-smoothing if we use the generalized cross-validation estimates of the bandwidth in our estimator.

Kernel estimator (9) is one of many estimators that use kernel functions as weights for the response values $Y_{1}, \ldots, Y_{n}$. This section gives the form and the intuition behind some common kernel-type regression estimators. As we shall see, each estimator performs best under specific conditions, and may be chosen depending on the type of design.

One generalization of estimator (9) is the Priestley-Chao estimator, introduced by Priestly and Chao (1972):

$$
f_{n, 1}(x)=\sum_{i=1}^{n}\left(x_{i}-x_{i-1}\right) K_{h}\left(x-x_{i}\right) Y_{i} .
$$

This estimator replaces the factor $1 / n$ in estimator (9) by the difference $x_{i}-x_{i-1}$, which is a value between 0 and 1 , with $x_{0}=0$ and $x_{n+1}=1$. In a region of sparse data, where the design points $x_{i}$ and $x_{i-1}$ are far apart, the $i$ th weighted response will have more impact on the estimator than the weighted response corresponding to a 
design point in a region with many observations, where $x_{i}$ and $x_{i-1}$ are closer together. As a result, the Priestly-Chao estimator will not emphasize responses coming from a region where observations are clustered together over responses from a region that is not as well-represented. This modification makes the Priestley-Chao estimator better suited for designs with unequally-spaced knots than the kernel estimator (9). The latter is a special case of estimator (10) when the design knots are equally-spaced. Another well-known kernel estimator is the Nadaya-Watson estimator introduced by Nadaraya (1964) and Watson (1964). This estimator is intuitively appealing because it is a weighted average of the responses with weights that add up to 1. Moreover, this estimator is an intuitive consequence of estimating the unknown conditional expectation function under random design. The Nadaraya-Watson estimator is given by

$$
f_{n, 2}(x)=\frac{\sum_{i=1}^{n} K_{h}\left(x-x_{i}\right) Y_{i}}{\sum_{j=1}^{n} K_{h}\left(x-x_{j}\right)}
$$

This estimator can be thought of as a local constant estimator because it essentially fits a constant in the neighbourhood around each point. Currently, the NadarayaWatson estimator is rarely used in practice due to its drawbacks. One defect of this estimator is its poor performance near the boundary of the observation interval, which can be improved by fitting a higher order polynomial around each point. Also, in a region of sparse data, it is possible for the denominator to be zero. Hence the Nadaraya-Watson estimator may be undefined in regions of sparse data. This is another serious drawback.

Local $p$ th order polynomial regression estimators are commonly used to fit a polynomial of order $p$ in the neigbourhood of each design point to obtain an estimate of 
the regression function at $x$. Suppose that $f$ is $p$-times differentiable at the point $x$. Then we can write Taylor's expansion of $f$ at a point $x_{i}$ in a neighbourhood around $x$. If we let $x_{i}-x=h$, and if we let $h \rightarrow 0$, this is given by

$$
\begin{aligned}
f\left(x_{i}\right) & =\sum_{j=0}^{\infty} \frac{f^{(j)}(x)\left(x_{i}-x\right)^{j}}{j !} \\
& =\sum_{j=0}^{p} \frac{f^{(j)}(x)\left(x_{i}-x\right)^{j}}{j !}+o\left(h^{p}\right) .
\end{aligned}
$$

Denote the vector of the first $p+1$ Taylor coefficients by

$$
\boldsymbol{\beta}=\left(f(x), f^{\prime}(x), \ldots, \frac{f^{(p)}(x)}{p !}\right)^{\top}
$$

Notice that the first element of $\boldsymbol{\beta}$ is just the regression function evaluated at the point $x$. Therefore, to obtain an estimate of $f(x)$, we may estimate the vector $\boldsymbol{\beta}$ by solving the weighted minimization problem

$$
\sum_{i=1}^{n} K_{h}\left(x-x_{i}\right)\left(Y_{i}-\sum_{j=0}^{p} \beta_{j}\left(x_{i}-x\right)^{j}\right)^{2} \rightarrow \min _{\boldsymbol{\beta}=\left(\beta_{0}, \ldots, \beta_{p}\right)^{\top}}
$$

where the kernel weights serve to control the contribution of different design points based on their distance from the point of evaluation $x$. The first element of the solution vector to this optimization problem is called the local polynomial estimator of order $p$ and it is given by the first element of the vector

$$
\hat{\boldsymbol{\beta}}=\left(\hat{\beta}_{0}, \ldots, \hat{\beta}_{p}\right)^{\top}=\left(\mathbf{X}^{\top} \mathbf{W} \mathbf{X}\right)^{-1} \mathbf{X}^{\top} \mathbf{W} \mathbf{Y}
$$


where $\mathbf{W}=\operatorname{diag}\left(K_{h}\left(x-x_{i}\right)\right), \mathbf{Y}=\left(Y_{1}, \ldots, Y_{n}\right)^{\top}$ and

$$
\mathbf{X}=\left(\begin{array}{cccc}
1 & \left(x_{1}-x\right) & \ldots & \left(x_{1}-x\right)^{p} \\
\vdots & \vdots & & \vdots \\
1 & \left(x_{n}-x\right) & \ldots & \left(x_{n}-x\right)^{p}
\end{array}\right)
$$

In other words, the vector $\hat{\boldsymbol{\beta}}$ is an estimator of the first $p+1$ coefficients obtained by fitting a polynomial of order $p$ around each design point, and the first element $\hat{\beta}_{0}$ is exactly the $p$ th order local polynomial estimator of $f$ :

$$
f_{n, 3}(x)=\hat{\beta}_{0}=\left[\left(\mathbf{X}^{\top} \mathbf{W X}\right)^{-1} \mathbf{X}^{\top} \mathbf{W} \mathbf{Y}\right]_{1}
$$

Generally, local polynomial estimators of odd degree have better end-point performance than those of even degree. Fan and Gijbels (1996) showed that increasing $p$ decreases the bias of the estimator while increasing the variance, because increasing the order also increases the number of coefficients to be estimated. From the definition of the local polynomial estimator it is clear that the Nadaraya-Watson estimator (11) is a 0th order local, or local constant, polynomial estimator. However, estimator (12) with $p>0$ is more flexible than the Nadaraya-Watson estimator and has better performance at the boundaries for odd $p$ (see Fan and Gijbels, 1996).

Another type of estimator is the convolution estimator given by the convolution of the kernel function with some function of the data. A well-known example is the estimator proposed by Gasser and Müller (1979). The Gasser-Müller estimator is essentially the convolution of the kernel function with a step function based on the data. Under the assumption that the observation interval is the interval $[0,1]$, the Gasser-Müller estimator can be written as 


$$
f_{n, 4}(x)=\sum_{i=1}^{n} Y_{i} \int_{s_{i-1}}^{s_{i}} K_{h}(x-y) d y
$$

where $s_{i}=\frac{x_{i+1}-x_{i}}{2}, s_{0}=0$ and $s_{n+1}=1$. Although convolution estimators are well suited to theoretical analysis, they present problems in high-dimensional regression estimation.

\subsection{Asymptotic Properties}

A popular criterion to judge the quality of estimators is the point-wise risk. The risk (mean squared error) of $f_{n}(x)$ as an estimator of $f(x)$ is defined by

$$
R_{n}(x)=\mathbf{E}_{f}\left(f(x)-f_{n}(x)\right)^{2}
$$

It represents the expected loss to the statistician who uses estimator $f_{n}$ to estimate the regression function $f$ at the point $x$. One advantage of using this criterion is that convergence of $R_{n}(x)$ to zero implies consistency of the estimator $f_{n}(x)$. Indeed, if

$$
\lim _{n \rightarrow \infty} R_{n}(x)=0
$$

then due to Chebyshev's inequality

$$
f_{n}(x) \stackrel{\mathbf{P}}{\longrightarrow} f(x), \quad n \rightarrow \infty
$$

i.e., $f_{n}(x)$ is a consistent estimator of $f(x)$.

The risk $R_{n}(x)$ can be broken up into a "variance term" and a "bias term" (sys- 
tematic error) as follows

$$
\begin{aligned}
R_{n}(x) & =\mathbf{E}_{f}\left(f(x)-f_{n}(x)\right)^{2} \\
& =\mathbf{E}_{f}\left(f_{n}(x)-\mathbf{E}_{f} f_{n}(x)\right)+\left(\mathbf{E}_{f} f_{n}(x)-f(x)\right)^{2} \\
& =\mathbf{E}_{f}\left(f_{n}(x)-\mathbf{E}_{f} f_{n}(x)\right)^{2}+\left(\mathbf{E}_{f} f_{n}(x)-f(x)\right)^{2} \\
& =\operatorname{Var}_{f}\left(f_{n}(x)\right)+\mathbf{b}_{n}^{2}(x)
\end{aligned}
$$

We shall now demonstrate that there is always a bias-variance tradeoff between these two terms: as variance decreases, bias increases at a known rate for various estimators. In the problem of finding a rate-optimal estimator of an unknown regression function, the key to minimizing the risk is to balance these terms optimally. We shall examine this problem in detail for the kernel estimator

$$
f_{n}(x)=n^{-1} \sum_{i=1}^{n} K_{h}\left(x-x_{i}\right) Y_{i}
$$

The asymptotic risk of $f_{n}(x)$ in the case of equally-spaced design points was first derived by Benedetti (1977). We shall informally present the ideas behind this derivation. The rigorous derivation is omitted for two reasons. First, informal discussion makes the basic ideas more transparent. Second, a rigorous proof would require exact smoothness conditions on $f$. Imposing such conditions would restrain our consideration to a specific functional class $\mathcal{F}=\{f\}$. 
For any $x \in[a, b]=[0,1]$, using the fact that $\mathbf{E} Y_{i}=f\left(x_{i}\right)$,

$$
\begin{aligned}
\mathrm{E} f_{n}(x) & =\mathbf{E}\left(n^{-1} \sum_{i=1}^{n} K_{h}\left(x-x_{i}\right) Y_{i}\right) \\
& =n^{-1} \sum_{i=1}^{n} K_{h}\left(x-x_{i}\right) \mathbf{E} Y_{i} \\
& =n^{-1} \sum_{i=1}^{n} K_{h}\left(x-x_{i}\right) f\left(x_{i}\right) .
\end{aligned}
$$

Therefore, the variance term is equal to

$$
\begin{aligned}
\operatorname{Var} f_{n}(x) & =\mathbf{E}\left(f_{n}(x)-\mathbf{E} f_{n}(x)\right)^{2} \\
& =\mathbf{E}\left(n^{-1} \sum_{i=1}^{n} K_{h}\left(x-x_{i}\right) Y_{i}-n^{-1} \sum_{i=1}^{n} K_{h}\left(x-x_{i}\right) f\left(x_{i}\right)\right)^{2} \\
& =\mathbf{E}\left(n^{-1} \sum_{i=1}^{n} K_{h}\left(x-x_{i}\right)\left(Y_{i}-f\left(x_{i}\right)\right)\right)^{2} \\
& =\mathbf{E}\left(n^{-1} \sum_{i=1}^{n} K_{h}\left(x-x_{i}\right) \varepsilon_{i}\right)^{2} \\
& =\operatorname{Var}\left(n^{-1} \sum_{i=1}^{n} K_{h}\left(x-x_{i}\right) \varepsilon_{i}\right)+\left[\mathbf{E}\left(n^{-1} \sum_{i=1}^{n} K_{h}\left(x-x_{i}\right) \varepsilon_{i}\right)\right]^{2}
\end{aligned}
$$

where $\mathbf{E}\left(n^{-1} \sum_{i=1}^{n} K_{h}\left(x-x_{i}\right) \varepsilon_{i}\right)=n^{-1} \sum_{i=1}^{n} K_{h}\left(x-x_{i}\right) \mathbf{E} \varepsilon_{i}=0$. From this, by the independence of $Y_{1}, \ldots, Y_{n}$,

$$
\begin{aligned}
\operatorname{Var} f_{n}(x) & =n^{-2} \sum_{i=1}^{n} \operatorname{Var}\left(K_{h}\left(x-x_{i}\right) \varepsilon_{i}\right) \\
& =n^{-2} \sum_{i=1}^{n} K_{h}^{2}\left(x-x_{i}\right) \operatorname{Var}\left(\varepsilon_{i}\right) \\
& =\sigma^{2} n^{-2} \sum_{i=1}^{n} K_{h}^{2}\left(x-x_{i}\right) .
\end{aligned}
$$

We are interested in the behavior of $\operatorname{Var} f_{n}(x)$ when $n$ is large. Recall that for any 
sufficiently smooth function $g$ we can write

$$
\int g(x)=\delta \sum_{k=1}^{n} g\left(x_{k}\right)+R
$$

Here $x_{0}=a, x_{n}=b, x_{k}=x_{0}+k \delta, k=1,2, \ldots, n$ are the interpolation nodes, $\delta=(b-a) / n$, and $R=O\left(n^{-1}\right)$, as $n \rightarrow \infty$, is the error of approximation (see Section 19.3.2, Bronshtein, et al., 1998).

We assume that $a=0$ and $b=1$, and therefore $\delta=n^{-1}$. For given $x \in[0,1]$ and $h$, putting $g(y)=K_{h}^{2}(x-y)$ and applying formula (14) we can continue, informally,

$$
\operatorname{Var} f_{n}(x)=\sigma^{2} n^{-2} \sum_{i=1}^{n} K_{h}\left(x-x_{i}\right)=\sigma^{2} n^{-1} \int_{0}^{1} K_{h}^{2}(x-u) d u+\ldots
$$

Recall that

$$
K_{h}(x)=h^{-1} K\left(h^{-1} x\right)
$$

Then making the substitution $v=h^{-1}(x-u)$, and letting $h \rightarrow \infty$, we obtain, informally,

$$
\operatorname{Var} f_{n}(x)=\sigma^{2}(n h)^{-1} \int_{-\infty}^{\infty} K^{2}(v) d v+\ldots
$$

Now let us analyze the systematic error, or bias term. For any $x \in[0,1]$, recalling that $\mathbf{E} Y_{i}=f\left(x_{i}\right)$ 


$$
\begin{aligned}
\mathbf{b}_{n}(x) & =\mathbf{E} f_{n}(x)-f(x) \\
& =\mathbf{E}\left(n^{-1} \sum_{i=1}^{n} K_{h}\left(x-x_{i}\right) Y_{i}\right)-f(x) \\
& =n^{-1} \sum_{i=1}^{n} K_{h}\left(x-x_{i}\right) \mathbf{E} Y_{i}-f(x) \\
& =n^{-1} \sum_{i=1}^{n} K_{h}\left(x-x_{i}\right) f\left(x_{i}\right)-f(x) .
\end{aligned}
$$

Applying (14), we can rewrite, informally,

$$
\mathbf{b}_{n}(x)=\int_{0}^{1} K_{h}(x-u) f(u) d u-f(x)+\ldots
$$

Now, by making the substitution $v=h^{-1}(u-x)$ and by using property (3), we obtain, as $n \rightarrow \infty$,

$$
\mathbf{b}_{n}(x)=\int_{-\infty}^{\infty} K(v)(f(x+h v)-f(x)) d v+\ldots
$$

Assuming that $f$ is at least two times differentiable at $x$,

$$
f(x+h v)-f(x)=f^{\prime}(x) h v+\frac{1}{2} f^{\prime \prime}(x) h^{2} v^{2}+o\left(h^{2}\right) .
$$

Therefore, taking into account (15), we can rewrite the bias $\mathbf{b}_{n}(x)$ in the form

$$
\mathbf{b}_{n}(x)=h f^{\prime}(x) \int_{-\infty}^{\infty} v K(v) d v+\frac{h^{2}}{2} f^{\prime \prime}(x) \int_{-\infty}^{\infty} v^{2} K(v) d v+\ldots
$$


where the first term is zero by property (4). Thus,

$$
\mathbf{b}_{n}(x)=\frac{h^{2}}{2} f^{\prime \prime}(x) \int_{-\infty}^{\infty} v^{2} K(v) d v+\ldots
$$

And therefore, informally, the squared bias is given by

$$
\mathrm{b}_{n}(x)=\frac{h^{4}}{4}\left(f^{\prime \prime}(x)\right)^{2}\left(\int_{-\infty}^{\infty} v^{2} K(v) d v\right)^{2}+\ldots
$$

This gives the approximate value of the risk for large $n$ in the form

$$
\begin{aligned}
R_{n}(x) & =\operatorname{Var}_{f} f_{n}(x)+\mathbf{b}_{n}^{2}(x) \\
& =\frac{\sigma^{2}}{n h} \int_{-\infty}^{\infty} K^{2}(v) d v+\frac{h^{4}}{4}\left(f^{\prime \prime}(x)\right)^{2}\left(\int_{-\infty}^{\infty} v^{2} K(v) d v\right)^{2}+\ldots
\end{aligned}
$$

If we assume, for example, that $f$ is twice continuously differentiable on the interval $[0,1]$, then the above derivation can be shown to be valid and, for any $x \in[0,1]$, the risk will be

$$
R_{n}(x)=O\left((n h)^{-1}\right)+O\left(h^{4}\right), \quad n \rightarrow \infty
$$

The first term of the point-wise risk represents the variance term and the second represents the squared bias term. This illustrates that the choice of the smoothing parameter $h$ is crucial: we would like to keep both terms small, but increasing $h$ will lower variance while increasing bias. This is often called the variance-bias tradeoff. The key is to chose $h$ to balance both terms as will be seen shortly. Müller (1988) showed that if $f \in C^{2}[0,1]$, then the risk of the Gasser-Müller estimator (13) of $f$ is 
given by

$$
\begin{aligned}
R_{n}(x) & =\frac{\sigma^{2} \int_{-\infty}^{\infty} K^{2}(u) d u}{n h}+O\left((n h)^{-2}\right)+\frac{h^{4}}{4}\left(f^{\prime \prime}(x)\right)^{2}\left(\int_{-\infty}^{\infty} v^{2} K(v) d v\right)^{2} \\
& +o\left(h^{4}\right)+O\left(n^{-2}\right)=O\left((n h)^{-1}\right)+O\left(h^{4}\right), \quad n \rightarrow \infty
\end{aligned}
$$

A similar representation for the risk of the local polynomial estimator was derived in Ruppert and Wand (1994). From this result one can obtain the asymptotic risk of the Nadaraya--Watson estimator by setting $p=0$.

Note that all the estimators considered here have asymptotic risk of the form $C_{1}(n h)^{-1}+C_{2} h^{4}$, where $C_{1}$ and $C_{2}$ are constants that depend on the estimated function $f$ and its estimator $f_{n}$. In other words, the order of magnitude of the variance term is $O\left((n h)^{-1}\right)$, and the order of magnitude of the bias is $O\left(h^{4}\right)$. In the problem of constructing a rate-optimal estimator of $f(x)$, one has to choose a bandwidth $h$ that minimizes the asymptotic risk up to a constant. This value of $h$ is called optimal bandwidth and is denoted by $h_{\text {opt }}$ :

$$
h_{\text {opt }}=\underset{h}{\operatorname{argmin}}\left\{C_{1} \frac{1}{n h}+C_{2} h^{4}\right\}
$$

This minimization problem is easily solved for

$$
h_{\text {opt }}=O\left(n^{-1 / 5}\right)
$$

For any $x \in[0,1]$, under the optimal bandwidth, the risk of the kernel estimators considered in this chapter converges (point-wisely) to zero at the rate $r_{n}=n^{-2 / 5}$ :

$$
\sup _{f \in \mathcal{F}_{2}} \mathbf{E}\left(n^{2 / 5}\left(f_{n}(x)-f(x)\right)\right)^{2}=O(1), \quad n \rightarrow \infty
$$


In other words, the point-wise quadratic risk of kernel estimator $f_{n}$ as an estimator of $f \in C^{2}[0,1]$ is $O\left(n^{-4 / 5}\right)$. Moreover, the rate $r_{n}=n^{-2 / 5}$ is know to be optimal.

Finally note that in some cases, kernel regression estimation is related to other types of nonparametric regression estimation. For example, in the following chapter, we will show that under some conditions, a kernel regression estimator is approximately equivalent to a type of orthogonal projection estimator. In Chapter 4, we will discuss a result by Silverman (1984) that under some conditions, a spline smoothing estimator is asymptotically equivalent to a kernel regression estimator that uses a so-called "spline kernel". 


\section{Orthogonal Series Estimation}

\subsection{Some Function Space Theory}

For the purpose of nonparamteric regression analysis, the true regression function $f$ is assumed to belong to some general functional class $\mathcal{F}$. $\mathcal{F}$ is often assumed to be a subset of $L_{2}[a, b]$, the class of all square integrable functions on the interval $[a, b]$. Let us examine this class and some of its useful properties.

Let $L_{2}[a, b]$ denote the set of functions $f:[a, b] \rightarrow \mathbb{R}$ such that $\int_{a}^{b} f^{2}(x) d x<\infty$. The scalar product between two functions $f$ and $g$ in $L_{2}[a, b]$ is

$$
(f, g)=\int_{a}^{b} f(x) g(x) d x
$$

and the norm of $f$ is

$$
\|f\|_{2}=(f, f)^{1 / 2}=\left(\int_{a}^{b} f^{2}(x) d x\right)^{1 / 2} .
$$

Note: Although elements of $L_{2}[a, b]$ are called functions, they are actually classes of equivalent functions. In other words, an element $f$ of $L_{2}[a, b]$ is $f=\left\{g: \int_{a}^{b} g^{2}(x) d x<\right.$ $\left.\infty,\|g-f\|_{2}=0\right\}$.

The functional space $L_{2}[a, b]$ is a vector space. Indeed, suppose that $f$ and $g$ are two arbitrary elements of $L_{2}[a, b]$. By applying Minkowski's inequality,

$$
\begin{aligned}
\|f+g\|_{2} & =\left(\int_{a}^{b}|f(x)+g(x)|^{2} d x\right)^{1 / 2} \\
& \leq\left(\int_{a}^{b}|f(x)|^{2} d x\right)^{1 / 2}+\left(\int_{a}^{b}|g(x)|^{2} d x\right)^{1 / 2}=\|f\|_{2}+\|g\|_{2}<\infty
\end{aligned}
$$


and for $c \in \mathbb{R}$,

$$
\begin{aligned}
\|c f\|_{2} & =\left(\int_{a}^{b}(c f(x))^{2} d x\right)^{1 / 2} \\
& \leq|c|\left(\int_{a}^{b} f(x)^{2} d x\right)^{1 / 2}=c\|f\|_{2}<\infty
\end{aligned}
$$

The proofs of the remaining 6 axioms of a vector space are trivial. Thus, $L_{2}[a, b]$ is a vector space. Therefore, intuitively, it should be possible to represent any element of this class as a linear combination of basis functions.

Definition 3.1. A sequence of functions $\phi_{0}, \phi_{1}, \ldots$ is called orthonormal with respect to the scalar product $(\cdot, \cdot)$ if for any $k, l=0,1, \ldots$,

$$
\left(\phi_{k}, \phi_{l}\right)=\delta_{k l}
$$

where

$$
\delta_{k l}=\left\{\begin{array}{cc}
1, & k=l \\
0, & k \neq l
\end{array}\right.
$$

is the Kronecker delta. The sequence is complete if $\left(f, \phi_{k}\right)=0$, for all $k$, implies that $f$ is the zero function in $L_{2}[a, b]$.

A complete, orthogonal set of functions in $L_{2}[a, b]$ forms a basis. More precisely, the following result holds (see, for example, Wasserman (2006), Sect. 7.2.) Theorem 3.1. If $f \in L_{2}[a, b]$ then

$$
f(x)=\sum_{k=0}^{\infty} c_{k} \phi_{k}(x)
$$

where $c_{k}=\left(f, \phi_{k}\right), k=0,1, \ldots$ are the Fourier coefficients of $f$ with respect to the 
sequence $\phi_{0}, \phi_{1}, \ldots$ Furthermore,

$$
\int_{a}^{b} f^{2}(x) d x=\sum_{k=0}^{\infty} c_{k}^{2}
$$

holds. This is known as Parseval's equality.

Expansion (16) is the Fourier series of $f$ with respect to the sequence $\phi_{0}, \phi_{1}, \ldots$ The equality sign in (16) means that $\int_{a}^{b}\left(f(x)-f_{N}(x)\right)^{2} d x \rightarrow 0$, as $N \rightarrow \infty$, where $f_{N}(x)=\sum_{k=0}^{N-1} c_{k} \phi_{k}(x)$ is the Nth partial sum of the Fourier series of function $f$. The $N$ th partial sum $f_{N}$ has the property of best approximation, i.e., for fixed $N, f_{N}(x)$ gives the smallest value of $\left\|f-\sum_{k=0}^{N-1} a_{k} \phi_{k}\right\|_{2}$ for all $\left(a_{0}, \ldots, a_{N-1}\right) \in \mathbb{R}^{N}$. In the following, we restrict our attention to sequences of functions $\left\{\phi_{k}\right\}_{k=0}^{\infty}$ that are both complete and orthonormal. This is known as a complete orthonormal system.

However, a problem arises if we choose to work with the functional class $L_{2}[a, b]$. Elements of $L_{2}[a, b]$ are difficult to evaluate at a point on the interval $[a, b]$. If two functions $f$ and $g$ in $L_{2}[a, b]$ have the property $\|f-g\|_{2}=0$, they are equal almost everywhere, but not necessarily everywhere. To overcome this difficulty, subsets of $L_{2}[a, b]$ will be examined that retain the useful properties of this functional space, yet do not have its difficulty of evaluation.

Let $C^{m}[a, b]$ be the set of all functions on the interval $[a, b]$ with $m$ continuous derivatives:

$$
C^{m}[a, b]=\left\{f: f^{(j)} \text { is continuous, } j=0, \ldots, m\right\}
$$

The symbol $C^{0}[a, b]$ denotes the set of all continuous functions on $[a, b]$. Also, let $C^{\infty}[a, b]$ be a set of all infinitely-differentiable functions on $[a, b]$. All functions continuous on a closed interval are bounded, and hence are square integrable. For any 
$m \geq 1$, we have

$$
C^{\infty}[a, b] \subset C^{m}[a, b] \subset C^{1}[a, b] \subset C^{0}[a, b] \subset L_{2}[a, b]
$$

Let $W_{2}^{m}[a, b]$ be the set of all functions on the interval $[a, b]$ with $(m-1)$ absolutely continuous derivatives, and with a square integrable $m$ th derivative. This is known as the mth order Sobolev space:

$W_{2}^{m}[a, b]=\left\{f: f^{(j)}\right.$ is absolutely continuous, $j=0, \ldots, m-1$, and $\left.f^{(m)} \in L_{2}[a, b]\right\}$

Thus, the 0th order Sobolev space $W_{2}^{0}[a, b]$ is simply the class of square integrable functions. Moreover, an element belonging to the $m$ th order Sobolev space is a subset of the set of all functions with $m$ continuous derivatives:

$$
C^{m}[a, b] \subset W_{2}^{m}[a, b] \subset C^{0}[a, b] \subset W_{2}^{0}[a, b]=L_{2}[a, b], \quad m \geq 0 .
$$

The subsets $C^{m}[a, b], m \geq 0$, and $W_{2}^{m}[a, b], m \geq 1$, contain only continuous functions. Hence two elements $f$ and $g$ in $C^{m}[a, b], m \geq 0$, or $W_{2}^{m}[a, b], m \geq 1$, such that $\|f-g\|_{2}=0$ must be equal, and thus point evaluation is possible. Moreover, these two functional classes share the useful properties of the space $L_{2}[a, b]$. Therefore, it is often convenient to assume that the regression function belongs to either $C^{m}[a, b]$, for some $m \geq 0$, or $W_{2}^{m}[a, b]$, for some $m \geq 1$.

Another useful functional space is a weighted $L_{2}$-space. For some non-negative function $\omega$ defined on $[a, b]$ and satisfying $\int_{a}^{b} \omega(x) d x<\infty$, denote by $L_{2}([a, b], \omega)$ the set of functions $f:[a, b] \rightarrow \mathbb{R}$ such that $\int_{a}^{b} f^{2}(x) \omega(x) d x<\infty$. The function $\omega$ is called the weight function. For two functions $f$ and $g$ in $L_{2}([a, b], \omega)$ the scalar 
product is defined by

$$
(f, g)=\int_{a}^{b} f(x) g(x) \omega(x) d x
$$

and the norm is defined by

$$
\|f\|=(f, f)^{1 / 2}=\left\{\int_{a}^{b} f^{2}(x) \omega(x) d x\right\}^{1 / 2}
$$

In the remainder of this chapter, it will be clear from the context whether the weighted norm or the regular unweighted norm is used.

\subsection{Two Types of Design and General Form of Projection Estimators}

Consider the discrete fixed design regression model

$$
Y_{i}=f\left(x_{i}\right)+\varepsilon_{i}, \quad i=1, \ldots, n,
$$

where $f$ is an unknown regression function that belongs to some known functional class $\mathcal{F}$. The class $\mathcal{F}$ is a subset of the $L_{2}$-space (either weighted or unweighted), $x_{i}$ are design points, or design knots, and $\varepsilon_{i}, i=1, \ldots, n$ are independent $N\left(0, \sigma^{2}\right)$ normal random errors. By Theorem 3.1, the function $f$ can be written as a Fourier series

$$
f(x)=\sum_{k=0}^{\infty} c_{k} \phi_{k}(x)
$$

where $\left\{\phi_{k}\right\}_{k=0}^{\infty}$ is a complete orthonormal system in $L_{2}[a, b]$ (or $L_{2}([a, b], \omega)$ ) and $c_{k}$ is the $k$ th Fourier coefficient given by $c_{k}=\left(f, \phi_{k}\right)$. 
To illustrate the idea of a projection estimator, we consider two types of design on a finite interval: uniform or equidistant and non-uniform.

Case 1. The uniform design on $[0,1]$ consists of equally spaced knots

$$
x_{i}=\frac{2 i-1}{2 n}, \quad i=1, \ldots, n
$$

Case 2. The uniform design on $[-1,1]$ consists of equally spaced knots

$$
x_{i}=\frac{2 i}{2 n+1}, \quad i=-n, \ldots,-1,0,1, \ldots, n
$$

(The corresponding regression model has the form

$$
Y_{i}=f\left(x_{i}\right)+\varepsilon_{i}, \quad i=-n, \ldots, n
$$

where $f$ is an unknown regression function and $\varepsilon_{i}$ are independent $N\left(0, \sigma^{2}\right)$ normal random errors.)

When estimating a regression function on a finite interval, the risk of the estimator becomes larger at the end-points relative to the interior of the observation interval. One way to increase the accuracy of estimation at the end-points is to use a nonuniform design. A popular choice for such a design is the Chebyshev design.

Case 3. The Chebyshev design is a non-uniform design on the interval $[-1,1]$ that consists of design knots

$$
x_{i}=\cos \frac{(2 i-1) \pi}{2 n}, \quad i=1, \ldots, n
$$

The Chebyshev knots $x_{i}$ are unequally spaced with spacing decreasing near the endpoints: they are the roots of Chebyshev polynomials of the first kind. Alternatively, 
the Chebyshev knots can be represented by projecting $n$ equally spaced points on a semi-circle on the $x$-axis (see Suetin, 1979, Section 3.1).

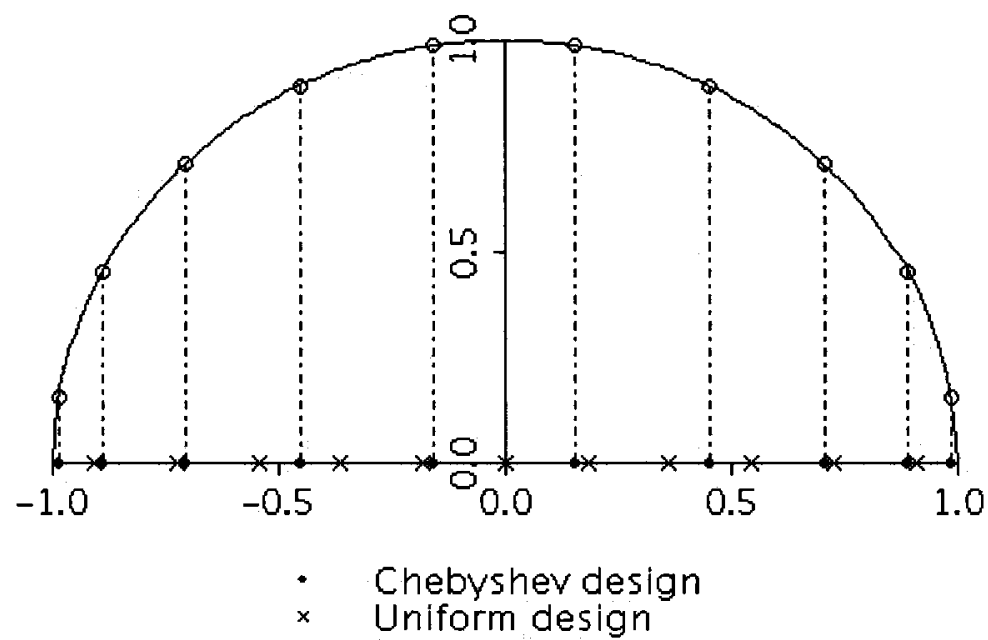

Figure 5: Chebyshev design on the interval $[-1,1]$ is equivalent to the projection on the $x$-axis of $n$ points that are equally-spaced on the unit semi-circle centered at the point $(0,0)$.

For further discussion, depending on the choice of a particular complete orthonormal system and the corresponding design $\left\{x_{i}\right\}$, we will need two discrete scalar products. For a given design $\left\{x_{i}\right\}$, define the discrete scalar product of two functions $f$ and $g$ to be either

$$
\langle f, g\rangle=\frac{1}{n} \sum_{i=1}^{n} f\left(x_{i}\right) g\left(x_{i}\right),
$$

or

$$
\langle f, g\rangle=\frac{2}{2 n+1} \sum_{i=-n}^{n} f\left(x_{i}\right) g\left(x_{i}\right) .
$$

The symbol $\langle\cdot, \cdot\rangle$ will be used to represent either the scalar product (20) or (21), depending on the context.

Definition 3.2. We say that two continuous functions $f$ and $g$ defined on $[a, b]$ are 
orthonormal with respect to the design $\left\{x_{i}\right\}$ if $\langle f, g\rangle=1$ when $f$ coincides with $g$ on $[a, b]$, and $\langle f, g\rangle=0$ otherwise.

A system of functions $\left\{\phi_{k}\right\}_{k=0}^{\infty}$ that is orthonormal with respect to both the scalar products $(\cdot, \cdot)$ and $\langle\cdot, \cdot\rangle$ is called double-orthonormal.

Orthogonal series estimators, sometimes called projection estimators, are a popular method of estimating nonparametric regression functions. This type of estimator was introduced in the field of density estimation by $\check{C}$ encov (1962). Further work on this subject was done by Wahba (1975) and Walter (1977). The idea behind a projection estimator is simple. It is well-known that for various classes of smooth functions contained in $L_{2}$, the Fourier coefficients in decomposition (16) decrease very fast. If $f$ is a function of finite smoothness, then the Fourier coefficient $c_{k}=\left(f, \phi_{k}\right)$ tends to zero as fast as a power of $k$ (see, for example, Section 707 of Fikhtengolts, 1963). If $f$ belongs to certain classes of infinitely-differentiable functions, then $c_{k}=$ $\left(f, \phi_{k}\right)$ decreases much faster, at an exponential rate (see, for example, page 140 of Timan, 1994). Therefore, one would only need to estimate those Fourier coefficient which could a priori be "large". Thus, a natural estimator of $f(x)$ based on the vector of observations $\mathbf{Y}=\left(Y_{1}, \ldots, Y_{n}\right)^{\top}$ is given by

$$
\tilde{f}_{n}(x)=\tilde{f}_{n}(x, \mathbf{Y})=\sum_{k=0}^{N-1} \tilde{c}_{k} \phi_{k}(x)
$$

where $\left\{\phi_{k}\right\}_{k=0}^{\infty}$ is a complete double-orthonormal system with respect to the design $\left\{x_{i}\right\}_{i=1}^{n}$, and, for $k \geq 0$,

$$
\tilde{c}_{k}:=\left\langle\mathbf{Y}, \phi_{k}\right\rangle=\frac{1}{n} \sum_{i=1}^{n} Y_{i} \phi_{k}\left(x_{i}\right)
$$

is an unbiased estimator of $c_{k}$. The unbiasedness of $c_{k}$ is obvious. Indeed, since 
$\left\{\phi_{k}\right\}_{k=0}^{\infty}$ is a complete double-orthonormal system in $L_{2}[a, b]$ with respect to the design $\left\{x_{i}\right\}_{i=1}^{n}$, it follows that

$$
\frac{1}{n} \sum_{i=1}^{n} \phi_{k}\left(x_{i}\right) \phi_{l}\left(x_{i}\right)=\delta_{k l}
$$

Using this and recalling that $f(x)=\sum_{k=0}^{\infty} c_{k} \phi_{k}(x)$, we have

$$
\begin{aligned}
\mathbf{E} \tilde{c}_{k} & =\mathbf{E}\left(\frac{1}{n} \sum_{i=1}^{n} Y_{i} \phi_{k}\left(x_{i}\right)\right)=\frac{1}{n} \sum_{i=1}^{n} \phi_{k}\left(x_{i}\right) \mathbf{E} Y_{i}=\frac{1}{n} \sum_{i=1}^{n} \phi_{k}\left(x_{i}\right) f\left(x_{i}\right) \\
& =\frac{1}{n} \sum_{i=1}^{n} \phi_{k}\left(x_{i}\right) \sum_{j=0}^{\infty} c_{j} \phi_{j}\left(x_{i}\right)=\frac{1}{n} \sum_{i=1}^{n} \sum_{j=0}^{\infty} c_{j} \phi_{k}\left(x_{i}\right) \phi_{j}\left(x_{i}\right) \\
& =\frac{1}{n} \sum_{i=1}^{n} c_{k} \phi_{k}\left(x_{i}\right) \phi_{k}\left(x_{i}\right)=c_{k} .
\end{aligned}
$$

In order to guarantee consistency of estimator (22), we assume that the parameter $N=N_{n}$ tends to infinity, but not as fast as $n$. That is, the orthogonal series estimator is consistent if

$$
N \rightarrow \infty \quad \text { and } \quad n^{-1} N \rightarrow 0, \quad \text { as } n \rightarrow \infty .
$$

Let us show this by deriving the risk of the orthogonal series estimator. Consider the risk, using the fact that $\mathbf{E} \tilde{c}_{k}=c_{k}$ :

$$
\begin{aligned}
\mathbf{E}\left(\tilde{f}_{n}(x)-f(x)\right)^{2} & =\mathbf{E}\left(\sum_{k=0}^{N-1}\left(\tilde{c}_{k}-c_{k}\right) \phi_{k}(x)-\sum_{k=N}^{\infty} c_{k} \phi_{k}(x)\right)^{2} \\
& =\mathbf{E}\left(\sum_{k=0}^{N-1}\left(\tilde{c}_{k}-c_{k}\right) \phi_{k}(x)\right)^{2}+\mathbf{E}\left(\sum_{k=N}^{\infty} c_{k} \phi_{k}(x)\right)^{2} \\
& =\mathbf{E} \sum_{k=0}^{N-1}\left(\tilde{c}_{k}-c_{k}\right)^{2} \phi_{k}^{2}(x)+b_{n}^{2}(x) \\
& =\text { main term }+ \text { bias term. }
\end{aligned}
$$


Using $\operatorname{Var} Y_{i}=\sigma^{2}$, independence of $Y_{1}, \ldots, Y_{n}$ and double-orthonormality of $\left\{\phi_{k}\right\}_{k=0}^{\infty}$, we obtain

$$
\begin{aligned}
\mathbf{E}\left(\tilde{c}_{k}-c_{k}\right)^{2} & =\operatorname{Var} \tilde{c}_{k}=\operatorname{Var}\left(\frac{1}{n} \sum_{i=1}^{n} Y_{i} \phi_{k}\left(x_{i}\right)\right) \\
& =\frac{\sigma^{2}}{n^{2}} \sum_{i=1}^{n} \phi_{k}^{2}\left(x_{i}\right)=\frac{\sigma^{2}}{n} .
\end{aligned}
$$

Therefore,

$$
\text { main term }=\frac{\sigma^{2}}{n} \sum_{k=0}^{N-1} \phi_{k}^{2}(x)=\sigma^{2} O\left(n^{-1} N\right)
$$

Now, since the basis functions $\phi_{k}, k=0,1, \ldots$ are bounded

$$
b_{n}^{2}(x) \leq\left(\sum_{k=N}^{\infty} c_{k} \phi_{k}(x)\right)^{2} \leq O(1)\left(\sum_{k=N}^{\infty}\left|c_{k}\right|\right)^{2}
$$

where, for example, $\left|c_{k}\right|=O\left(\frac{1}{k^{m+1}}\right)$ provided $\left|f^{(m)}(x)\right| \leq M, x \in[a, b]$ (see Section 707 of Fikhtengolts, 1963). In this case,

$$
b_{n}^{2}(x) \leq O(1)\left(\int_{N}^{\infty} \frac{d x}{x^{m+1}}\right)^{2}=O\left(\frac{1}{N^{2 m}}\right)
$$

Thus,

$$
\text { Risk } \leq O\left(n^{-1} N\right)+O\left(N^{-2 m}\right)
$$

This representation shows that if $N$ grows at a slower rate than $n$, then $\tilde{f}_{n}(x)$ is a consistent estimator of $f$. Optimizing the function

$$
C_{1} \frac{N}{n}+C_{2} N^{-2 m}
$$


with respect to $N$ gives

$$
N_{o p t}=O\left(n^{1 /(2 m+1)}\right)
$$

and the corresponding risk is

$$
\text { Risk } \leq O\left(n^{-2 m /(2 m+1)}\right)
$$

Thus, by choosing the number of terms in the estimator to be of order $n^{1 /(2 m+1)}$ the risk can be made to decay to zero at the rate $n^{-m /(2 m+1)}$ for a regression function $f$ satisfying $\left|f^{(m)}(x)\right| \leq M$ for all $x \in[a, b]$.

Note also that projection estimator (22) is asymptotically unbiased. Indeed, for every $x \in[a, b]$

$$
\begin{aligned}
\mathbf{E} \tilde{f}_{n}(x) & =\mathbf{E}\left(\sum_{k=0}^{N-1} \tilde{c}_{k} \phi_{k}(x)\right)=\mathbf{E}\left(\sum_{k=0}^{N-1} \frac{1}{n} \sum_{i=1}^{n} Y_{i} \phi_{k}\left(x_{i}\right) \phi_{k}(x)\right) \\
& =\sum_{k=0}^{N-1} \frac{1}{n} \sum_{i=1}^{n} \mathbf{E}\left(Y_{i}\right) \phi_{k}\left(x_{i}\right) \phi_{k}(x)=\sum_{k=0}^{N-1} \phi_{k}(x) \frac{1}{n} \sum_{i=1}^{n}\left(\sum_{j=0}^{\infty} c_{j} \phi_{j}\left(x_{i}\right)\right) \phi_{k}\left(x_{i}\right) \\
& =\sum_{k=0}^{N-1} \phi_{k}(x) \sum_{j=0}^{\infty} c_{j}\left(\frac{1}{n} \sum_{i=1}^{n} \phi_{j}\left(x_{i}\right) \phi_{k}\left(x_{i}\right)\right)=\sum_{k=0}^{N-1} \phi_{k}(x) \sum_{j=0}^{\infty} c_{j} \delta_{j k} \\
& =\sum_{k=0}^{N-1} c_{k} \phi_{k}(x) .
\end{aligned}
$$

Then, recalling that $N \rightarrow \infty$ as $n \rightarrow \infty$, we get

$$
\lim _{n \rightarrow \infty} \mathbf{E} \tilde{f}_{n}(x)=\lim _{n \rightarrow \infty} \sum_{k=0}^{N-1} c_{k} \phi_{k}(x)=\sum_{k=0}^{\infty} c_{k} \phi_{k}(x)=f(x)
$$

That is, for large numbers $n$ of design points, $\tilde{f}_{n}(x)$ is nearly an unbiased estimator of $f(x)$. 
The goal of the statistician is to choose a value of $N$ that will lead to the "best" possible estimator $\tilde{f}_{n}$ from a wide class of estimators of $f$. Methods of choosing $N$ in practice include the adaptive methods of cross-validation and generalized crossvalidation, which will be discussed in Chapter 5 .

\subsection{Projection Estimators Corresponding to Different De- signs}

Case 1. When dealing with the uniform design on $[0,1]$, we consider the system of normalized cosine polynomials $\left\{q_{k}(x)\right\}_{k=0}^{\infty}$ defined by

$$
q_{k}(x)= \begin{cases}1 & , k=0 \\ \sqrt{2} \cos (k \pi x) & , k=1,2, \ldots\end{cases}
$$

Clearly, each element of the system $\left\{q_{k}(x)\right\}_{k=0}^{\infty}$ belongs to $L_{2}[0,1]$. Moreover, the following result holds true.

Lemma 3.1. The system $\left\{q_{k}\right\}_{k=0}^{\infty}$ is complete and orthonormal with respect to the scalar product

$$
(f, g)=\int_{0}^{1} f(x) g(x) d x .
$$

It is also orthonormal over the uniform design (17), i.e.,

$$
\left\langle q_{k}, q_{l}\right\rangle=\frac{1}{n} \sum_{i=1}^{n} q_{k}\left(x_{i}\right) q_{l}\left(x_{i}\right)=\delta_{k l}, \quad k, l=0,1, \ldots
$$

Proof. Orthonormality of the cosine polynomial system is trivial. For $k=1,2 \ldots$,

$$
\left\|q_{k}\right\|^{2}=2 \int_{0}^{1} \cos ^{2}(k \pi x) d x=2\left(\frac{x}{2}+\left.\frac{\sin (2 k \pi x)}{4 k \pi}\right|_{0} ^{1}\right)=1,
$$


and for $k=0$,

$$
\left\|q_{0}\right\|^{2}=\int_{0}^{1} 1^{2} d x=1
$$

In the following calculations we use the trigonometric identity,

$$
\cos x+\cos y=2 \cos \frac{x+y}{2} \cos \frac{x-y}{2}
$$

For positive integers $k \neq l$, using identity (23)

$$
\begin{aligned}
\left(q_{k}, q_{l}\right) & =2 \int_{0}^{1} \cos (k \pi x) \cos (l \pi x) d x \\
& =\left.2\left(\frac{\sin [(k \pi-l \pi) x]}{2(k \pi-l \pi)}+\frac{\sin [(k \pi+l \pi) x]}{2(k \pi+l \pi)}\right)\right|_{0} ^{1} \\
& =\frac{\sin [(k-l) \pi]}{(k-l) \pi}+\frac{\sin [(k+l) \pi]}{(k+l) \pi} \\
& =0 .
\end{aligned}
$$

Also, for $k=0$ and $l=1,2, \ldots$,

$$
\left(q_{0}, q_{l}\right)=\sqrt{2} \int_{0}^{1} \cos (l \pi x) d x=\left.\frac{\sqrt{2} \sin (l \pi x)}{l \pi}\right|_{0} ^{1}=0 .
$$

Completeness of the cosine system $\left\{q_{k}\right\}_{k=0}^{\infty}$ in the space $L_{2}[0,1]$ is a well-known result in Functional Analysis. As the exact reference is not easily available, we will derive it from the fact that $\left\{e^{i k x}\right\}_{k \in \mathbb{Z}}$ is complete in $L_{2}[-\pi, \pi]$ (see, for example, Vretblad, 2003, Section 5.4.)

First, let us show that the system $\{\cos (k x)\}_{k=0}^{\infty}$ is complete in $L_{2}[0, \pi]$, i.e., that the equality $\int_{0}^{\pi} g(x) \cos (k x) d x=0$ for $k=0,1, \ldots$ implies that $g(x)=0$ almost 
everywhere. By the symmetry of $\cos x$ about zero,

$$
\int_{0}^{\pi} g(x) \cos (k x) d x=\int_{-\pi}^{0} g(-x) \cos (k x) d y
$$

Put

$$
G(x)= \begin{cases}g(x) & , \quad x \in[0, \pi], \\ g(-x), & x \in[-\pi, 0] .\end{cases}
$$

Then using the identity $\cos (k x)=\left(e^{i k x}+e^{-i k x}\right) / 2$, and the symmetry of $G(x)$, we get

$$
\begin{aligned}
& 2 \int_{0}^{\pi} g(x) \cos (k x) d x=\int_{-\pi}^{0} g(-x) \cos (k x) d x+\int_{0}^{\pi} g(x) \cos (k x) d x \\
& =\int_{-\pi}^{0} G(x) \cos (k x) d x+\int_{0}^{\pi} G(x) \cos (k x) d x=\int_{-\pi}^{\pi} G(x) \cos (k x) d x \\
& =\frac{1}{2} \int_{-\pi}^{\pi} G(x) e^{i k x} d x-\frac{1}{2} \int_{\pi}^{-\pi} G(-x) e^{i k x} d x=\int_{-\pi}^{\pi} G(x) e^{i k x} d x .
\end{aligned}
$$

Therefore, due to the completeness of the system $\left\{e^{i k x}\right\}_{k \in \mathbb{Z}}$, equality

$$
\int_{0}^{\pi} g(x) \cos (k x) d x=0
$$

implies $g(x)=0$ for almost all $x \in[0, \pi]$. So, the system $\{\cos (k t)\}_{k=0}^{\infty}$ is complete in $L_{2}[0, \pi]$. The completentness of $\{\sqrt{2} \cos (\pi k x)\}_{k=0}^{\infty}$ in $L_{2}[0,1]$ is now an immediate consequence of the identity

$$
\sqrt{2} \int_{0}^{\pi} g(x) \cos (k x) d x=\frac{1}{\pi} \int_{0}^{1} g(\pi y) \sqrt{2} \cos (k \pi y) d y
$$

Next, let us show that the cosine polynomial system $\left\{q_{k}\right\}_{k=0}^{\infty}$ is orthonormal over the 
uniform design (17). For positive integers $k \neq l$, using the trigonometric identity (see Gradshteyn and Ryzhik, 1979, 1.342.4)

$$
\sum_{k=1}^{n} \cos (2 k-1) x=\frac{\sin (2 n x)}{2 \sin x}
$$

and the identity

$$
\cos x \cos y=\frac{1}{2}(\cos (x+y)+\cos (x-y))
$$

we get

$$
\begin{aligned}
\left\langle q_{k}, q_{l}\right\rangle & =\frac{1}{n} \sum_{i=1}^{n} \sqrt{2} \cos \left(k \pi x_{i}\right) \sqrt{2} \cos \left(l \pi x_{i}\right) \\
& =\frac{2}{n} \sum_{i=1}^{n} \cos \left(\frac{k \pi(2 i-1)}{2 n}\right) \cos \left(\frac{l \pi(2 i-1)}{2 n}\right) \\
& =\frac{2}{n} \sum_{i=1}^{n} \frac{1}{2}\left[\cos \left(\frac{\pi(k+l)(2 i-1)}{2 n}\right)+\cos \left(\frac{\pi(k-l)(2 i-1)}{2 n}\right)\right] \\
& =\frac{1}{n} \sum_{i=1}^{n} \cos \left(\frac{\pi(k+l)(2 i-1)}{2 n}\right)+\frac{1}{n} \sum_{i=1}^{n} \cos \left(\frac{\pi(k-l)(2 i-1)}{2 n}\right) \\
& =\frac{1}{2 n} \frac{\sin (k+l) \pi}{\sin \frac{(k+l) \pi}{2 n}}+\frac{1}{2 n} \frac{\sin (k-l) \pi}{\sin \frac{(k-l) \pi}{2 n}}=0 .
\end{aligned}
$$

For $k=0$ and $l=1,2 \ldots$, due to (24), we obtain

$$
\begin{aligned}
\left\langle q_{0}, q_{l}\right\rangle & =\frac{1}{n} \sum_{i=1}^{n} q_{0}\left(x_{i}\right) q_{l}\left(x_{i}\right)=\frac{\sqrt{2}}{n} \sum_{i=1}^{n} \cos \left(\frac{l \pi(2 i-1)}{2 n}\right) \\
& =\frac{1}{\sqrt{2} n} \frac{\sin (l \pi)}{\sin \left(\frac{l \pi}{2 n}\right)}=0 .
\end{aligned}
$$

The above covers all the cases where $k \neq l$. The normalization condition $\left\langle q_{k}, q_{k}\right\rangle^{1 / 2}=$ 
$\left\|q_{k}\right\|=1$ is also easy to verify. For $k=l \neq 0$, using the trigonometric identity

$$
\cos ^{2} x=\frac{\cos 2 x+1}{2}
$$

we obtain

$$
\begin{aligned}
\left\langle q_{k}, q_{k}\right\rangle & =\frac{1}{n} \sum_{i=1}^{n} \sqrt{2} \cos \left(k \pi x_{i}\right) \sqrt{2} \cos \left(k \pi x_{i}\right)=\frac{2}{n} \sum_{i=1}^{n} \cos ^{2}\left(\frac{k \pi(2 i-1)}{2 n}\right) \\
& =\frac{1}{n} \sum_{i=1}^{n}\left(1+\cos \left(\frac{2 l \pi(2 i-1)}{2 n}\right)\right)=1+\frac{1}{n} \sum_{i=1}^{n} \cos \left(\frac{l \pi(2 i-1)}{n}\right) \\
& =1+\frac{1}{n} \frac{\sin (2 l \pi)}{2 \sin \left(\frac{l \pi}{n}\right)}=1 .
\end{aligned}
$$

For $k=l=0$,

$$
\left\langle q_{0}, q_{0}\right\rangle=\frac{1}{n} \sum_{i=1}^{n} q_{0}\left(x_{i}\right) q_{0}\left(x_{i}\right)=\frac{1}{n} \sum_{i=1}^{n} 1^{2}=1 .
$$

Thus, we have shown that the cosine polynomial system $\left\{q_{k}\right\}_{k=0}^{\infty}$ is orthonormal with respect to the design knots $x_{i}=\frac{2 i-1}{2 n}, i=1, \ldots, n$. The proof of Lemma 3.1 is complete.

Case 2. When dealing with the uniform design on $[-1,1]$, a natural choice for basis is the system of normalized trigonometric polynomials $\left\{h_{k}(x)\right\}_{k \in \mathbb{Z}}$ :

$$
h_{k}(x)= \begin{cases}\cos (k \pi x) & , k<0 \\ 1 / \sqrt{2} & , k=0 \\ \sin (k \pi x) & , k>0 .\end{cases}
$$

Lemma 3.2. The system $\left\{h_{k}\right\}_{k \in \mathbb{Z}}$ is complete and orthonormal with respect to the 
scalar product

$$
(f, g)=\int_{-1}^{1} f(x) g(x) d x
$$

Moreover, it is orthonormal over the uniform design (18), i.e.,

$$
\left\langle h_{k}, h_{l}\right\rangle=\frac{2}{2 n+1} \sum_{i=-n}^{n} h_{k}\left(x_{i}\right) h_{l}\left(x_{i}\right)=\delta_{k l}, \quad k, l \in \mathbb{Z}
$$

Proof. The trigonometric system $\left\{h_{k}(x)\right\}_{k \in \mathbb{Z}}$ is complete in $L_{2}[-1,1]$ (see, for example, Vretblad, 2003, Section 5.4.). Orthonormality of $\left\{h_{k}(x)\right\}_{k \in \mathbb{Z}}$ with respect to the scalar product $(f, g)$ is easy to check. Indeed, for negative integers $k \neq l$, using identity (25), we have

$$
\begin{aligned}
\left(h_{k}, h_{l}\right) & =\int_{-1}^{1} \cos (k \pi x) \cos (l \pi x) d x=\frac{1}{2} \int_{-1}^{1}(\cos ((k+l) \pi x)+\cos ((k-l) \pi x)) d x \\
& =\left.\left(\frac{\sin (k+l) \pi x}{2(k+l) \pi}+\frac{\sin (k-l) \pi x}{2(k-l) \pi}\right)\right|_{-1} ^{1}=0 .
\end{aligned}
$$

For positive integers $k \neq l$, using the fact that

$$
\sin x \sin y=\frac{1}{2}(\cos (x-y)-\cos (x+y)),
$$

we get

$$
\begin{aligned}
\left(h_{k}, h_{l}\right) & =\int_{-1}^{1} \sin (k \pi x) \sin (l \pi x) d x=\frac{1}{2} \int_{-1}^{1}(\cos (k-l) \pi x-\cos (k+l) \pi x) d x \\
& =\left.\left(\frac{\sin (k-l) \pi x}{2(k-l) \pi}-\frac{\sin (k+l) \pi x}{2(k+l) \pi}\right)\right|_{-1} ^{1}=0 .
\end{aligned}
$$


For positive integer $k$ and negative integer $l$, using

$$
\sin x \cos y=\frac{1}{2}(\sin (x+y)+\sin (x-y))
$$

we obtain

$$
\begin{aligned}
\left(h_{k}, h_{l}\right) & =\int_{-1}^{1} \sin (k \pi x) \cos (l \pi x) d x=\frac{1}{2} \int_{-1}^{1}(\sin (k+l) \pi x+\sin (k-l) \pi x) d x \\
& =\left.\left(-\frac{\cos (k-l) \pi x}{2(k-l) \pi}-\frac{\cos (k+l) \pi x}{2(k+l) \pi}\right)\right|_{-1} ^{1}=0
\end{aligned}
$$

Next, for $k=0$ and a negative integer $l$,

$$
\left(h_{0}, h_{l}\right)=\int_{-1}^{1} \frac{1}{\sqrt{2}} \cos (l \pi x) d x=\left.\frac{\sin (l \pi x)}{\sqrt{2} l \pi}\right|_{-1} ^{1}=0
$$

Similarly, for $k=0$ and a positive integer $l$,

$$
\left(h_{0}, h_{l}\right)=\int_{-1}^{1} \frac{1}{\sqrt{2}} \sin (l \pi x) d x=\left.\frac{-\cos (l \pi x)}{\sqrt{2} l \pi}\right|_{-1} ^{1}=0
$$

The above covers all the cases where $k \neq l$. Therefore, $\left(h_{k}, h_{l}\right)=0$ holds for any $k \in \mathbb{Z}$ and $l \in \mathbb{Z}$ such that $k \neq l$. Next, we show that each trigonometric polynomial is normalized so that $\left(h_{k}, h_{k}\right)^{1 / 2}=\left\|h_{k}\right\|=1$. For negative $k \neq 0$,

$$
\left\|h_{k}\right\|=\left(h_{k}, h_{k}\right)^{1 / 2}=\left[\int_{-1}^{1} \cos ^{2}(k \pi x) d x\right]^{1 / 2}=\left[\left.\left(\frac{x}{2}+\frac{\sin (2 k \pi x)}{4 k \pi}\right)\right|_{-1} ^{1}\right]^{1 / 2}=1
$$

For positive $k \neq 0$,

$$
\left\|h_{k}\right\|=\left(h_{k}, h_{k}\right)^{1 / 2}=\left[\int_{-1}^{1} \sin ^{2}(k \pi x) d x\right]^{1 / 2}=\left[\left.\left(\frac{x}{2}-\frac{\sin (2 k \pi x)}{4 k \pi}\right)\right|_{-1} ^{1}\right]^{1 / 2}=1
$$


And for $k=0$,

$$
\left\|h_{0}\right\|=\left(h_{0}, h_{0}\right)^{1 / 2}=\left[\int_{-1}^{1}(1 / \sqrt{2})^{2} d x\right]^{1 / 2}=1 .
$$

Thus, the trigonometric polynomial system $\left\{h_{k}(x)\right\}_{k \in \mathbb{Z}}$ is orthonormal with respect to the scalar product $(f, g)=\int_{-1}^{1} f(x) g(x) d x$.

Now, let us show that the system $\left\{h_{k}(x)\right\}_{k \in \mathbb{Z}}$ is orthonormnal with respect to the equidistant design knots $x_{i}=\frac{2 i}{2 n+1}, i=-n, \ldots, n$. First, we must check the orthogonality of $\left\{h_{k}(x)\right\}_{k \in \mathbb{Z}}$. Noting that $\sin x$ is an odd function, we have

$$
\sum_{i=-n}^{n} \sin \left(k \pi x_{i}\right)=0
$$

Also, since $\cos (x)$ is an even function, for any $k \neq 0$, due to the identity (see Gradshteyn and Ryzhik, 1979, 1.342.2)

$$
\sum_{k=0}^{n} \cos k x=\frac{1}{2}+\frac{\sin \left(n+\frac{1}{2}\right) x}{2 \sin \frac{x}{2}}
$$

we get,

$$
\begin{aligned}
\sum_{i=-n}^{n} \cos \left(k \pi x_{i}\right) & =\sum_{i=-n}^{n} \cos \left(\frac{2 k \pi i}{2 n+1}\right)=2 \sum_{i=0}^{n} \cos \left(\frac{2 k \pi i}{2 n+1}\right)-\cos (0) \\
& =2\left(\frac{1}{2}+\frac{\sin \left(n+\frac{1}{2}\right)\left(\frac{2 k \pi}{2 n+1}\right)}{2 \sin \frac{k \pi}{2 n+1}}\right)-1=\frac{\sin (k \pi)}{\sin \frac{k \pi}{2 n+1}}=0
\end{aligned}
$$

Thus, for $k \neq 0$,

$$
\sum_{i=-n}^{n} \cos \left(k \pi x_{i}\right)=0
$$


By $(25)\left\{h_{k}(x)\right\}_{k \in \mathbb{Z}}$ for negative $k$ and $l$,

$$
\begin{aligned}
\left\langle h_{k}, h_{l}\right\rangle & =\frac{2}{2 n+1} \sum_{i=-n}^{n} \cos \left(k \pi x_{i}\right) \cos \left(l \pi x_{i}\right) \\
& =\frac{1}{2 n+1} \sum_{i=-n}^{n} \cos \left((k+l) \pi x_{i}\right)+\frac{1}{2 n+1} \sum_{i=-n}^{n} \cos \left((k-l) \pi x_{i}\right) \\
& = \begin{cases}0, & k \neq l, \\
1, & k=l,\end{cases}
\end{aligned}
$$

where the last equality is due to (29). Next, applying (27), for positive $k$ an $l$,

$$
\begin{aligned}
\left\langle h_{k}, h_{l}\right\rangle & =\frac{2}{2 n+1} \sum_{i=-n}^{n} \sin \left(k \pi x_{i}\right) \sin \left(l \pi x_{i}\right) \\
& =\frac{1}{2 n+1} \sum_{i=-n}^{n} \cos ((k-l) \pi x)-\frac{1}{2 n+1} \sum_{i=-n}^{n} \cos ((k+l) \pi x) \\
& = \begin{cases}0, & k \neq l, \\
1, & k=l\end{cases}
\end{aligned}
$$

where the last equality is due to (29). Also, using (28), for negative $k$ and positive $l$,

$$
\begin{aligned}
\left\langle h_{k}, h_{l}\right\rangle & =\frac{2}{2 n+1} \sum_{i=-n}^{n} \cos \left(k \pi x_{i}\right) \sin \left(l \pi x_{i}\right) \\
& =\frac{1}{2 n+1} \sum_{i=-n}^{n} \sin \left((k+l) \pi x_{i}\right)+\frac{1}{2 n+1} \sum_{i=-n}^{n} \sin \left((k-l) \pi x_{i}\right) \\
& = \begin{cases}0, & k \neq l, \\
1, & k=l,\end{cases}
\end{aligned}
$$


where the last equality is due to (29). Moreover, for $k=0$ and $l$ negative,

$$
\left\langle h_{k}, h_{l}\right\rangle=\frac{2}{2 n+1} \sum_{i=-n}^{n} \frac{1}{\sqrt{2}} \cos \left(l \pi x_{i}\right)=\frac{1}{\sqrt{2}\left(n+\frac{1}{2}\right)} \sum_{i=-n}^{n} \cos \left(\frac{2 l \pi i}{2 n+1}\right)=0 .
$$

Similarly, for $k=0$ and $l$ positive,

$$
\left\langle h_{k}, h_{l}\right\rangle=\frac{2}{2 n+1} \sum_{i=-n}^{n} \frac{1}{\sqrt{2}} \sin \left(l \pi x_{i}\right)=\frac{1}{\sqrt{2}\left(n+\frac{1}{2}\right)} \sum_{i=-n}^{n} \sin \left(\frac{2 l \pi i}{2 n+1}\right)=0 .
$$

Finally,

$$
\left\langle h_{0}, h_{0}\right\rangle=\frac{2}{2 n+1} \sum_{i=-n}^{n}(1 / \sqrt{2})^{2}=1 \text {. }
$$

The proof Lemma 3.2 is complete.

Case 3. In the case of Chebyshev design, the system $\left\{t_{k}\right\}_{k=0}^{\infty}$ of normalized Chebyshev polynomials is used. These polynomials are defined as follows:

$$
t_{k}(x)= \begin{cases}1 & , k=0 \\ \sqrt{2} \cos (k \arccos x) & , k=1,2, \ldots\end{cases}
$$

Each element of this system belongs to the weighted space $L_{2}([-1,1], \omega)$ with weight function $\omega(x)=1 / \sqrt{1-x^{2}}$.

Lemma 3.3. The system $\left\{t_{k}\right\}_{k=0}^{\infty}$ is complete and orthonormal with respect to the scalar product

$$
(f, g)=\frac{1}{\pi} \int_{-1}^{1} \frac{f(x) g(x)}{\sqrt{1-x^{2}}} d x
$$

Moreover, the polynomials $t_{k}(x), k=0,1, \ldots$ are orthonormal over the Chebyshev 
design (19), i.e.,

$$
\left\langle t_{k}, t_{l}\right\rangle=\frac{1}{n} \sum_{i=1}^{n} t_{k}\left(x_{i}\right) t_{l}\left(x_{i}\right)=\delta_{k l}, \quad k, l=0,1, \ldots
$$

Proof. First, we show that the Chebyshev polynomials form an orthonormal system in the weighted $L_{2}$-space $L_{2}\left([-1,1],\left(1-x^{2}\right)^{-1 / 2}\right)$. For any positive integer $k$, using identity (26),

$$
\begin{aligned}
\left\|t_{k}\right\|^{2} & =\frac{1}{\pi} \int_{-1}^{1} \frac{t_{k}^{2}(x)}{\sqrt{1-x^{2}}} d x=\frac{2}{\pi} \int_{-1}^{1} \frac{\cos ^{2}(k \arccos x)}{\sqrt{1-x^{2}}} d x \\
& =\frac{1}{\pi} \int_{-1}^{1} \frac{1+\cos (2 k \arccos x)}{\sqrt{1-x^{2}}} d x \\
& =\frac{1}{\pi} \int_{-1}^{1} \frac{1}{\sqrt{1-x^{2}}} d x+\frac{1}{\pi} \int_{-1}^{1} \frac{\cos (2 k \arccos x)}{\sqrt{1-x^{2}}} d x \\
& =\left.\frac{-\arccos x}{\pi}\right|_{-1} ^{1}-\frac{1}{2 k \pi} \int_{-2 k \pi}^{0} \cos u d u \\
& =\left.\frac{-\arccos x}{\pi}\right|_{-1} ^{1}-\left.\frac{1}{2 k \pi} \sin u\right|_{-2 k \pi} ^{0}=1 .
\end{aligned}
$$

For $k=0$,

$$
\left\|t_{0}\right\|^{2}=\frac{1}{\pi} \int_{-1}^{1} \frac{1^{2}}{\sqrt{1-x^{2}}} d x=\frac{1}{\pi} \int_{-1}^{1} \frac{1}{\sqrt{1-x^{2}}} d x=\left.\frac{1}{\pi} \arccos (x)\right|_{-1} ^{1}=1 .
$$


Next, for positive integers $k \neq l$, by identity $(25)$,

$$
\begin{aligned}
\left(t_{k}, t_{l}\right) & =\frac{2}{\pi} \int_{-1}^{1} \frac{\cos (k \arccos x) \cos (l \arccos x)}{\sqrt{1-x^{2}}} d x \\
& =\frac{1}{\pi} \int_{-1}^{1} \frac{\cos ((k+l) \arccos x)+\cos ((k-l) \arccos x)}{\sqrt{1-x^{2}}} d x \\
& =\frac{1}{\pi} \int_{-1}^{1} \frac{\cos ((k+l) \arccos x)}{\sqrt{1-x^{2}}} d x+\frac{1}{\pi} \int_{-1}^{1} \frac{\cos ((k-l) \arccos x)}{\sqrt{1-x^{2}}} d x \\
& =-\left.\frac{\sin u}{\pi(k+l)}\right|_{(k+l) \pi} ^{0}-\left.\frac{\sin u}{\pi(k-l)}\right|_{(k+l) \pi} ^{0}=0 .
\end{aligned}
$$

Also, for $k=0$ and $l=1,2, \ldots$,

$$
\left(t_{0}, t_{l}\right)=\frac{\sqrt{2}}{\pi} \int_{-1}^{1} \frac{\cos (l \arccos x)}{\sqrt{1-x^{2}}} d x=-\left.\frac{\sin u}{l \pi}\right|_{l \pi} ^{0}=0
$$

The above covers all the cases where $k \neq l$. Therefore, the system $\left\{t_{k}(x)\right\}_{k=0}^{\infty}$ is orthonormal with respect to the scalar product $(f, g)=\pi^{-1} \int_{-1}^{1} \frac{f(x) g(x)}{\sqrt{1-x^{2}}} d x$.

Next, we will verify that the Chebyshev system $\left\{t_{k}\right\}_{k=0}^{\infty}$ is complete in the weighted $L_{2}$-space. For this, assume that $g(x) \in L_{2}([-1,1], \omega)$ satisfies

$$
\int_{-1}^{1} \frac{g(x) \cos (k \arccos x)}{\sqrt{1-x^{2}}} d x=0, \quad k=0,1, \ldots
$$

Using the change of variable $x=\cos y$, for $k=0,1 \ldots$,

$$
0=\int_{-1}^{1} \frac{g(x) \cos (k \arccos x)}{\sqrt{1-x^{2}}} d x=-\int_{0}^{\pi} g(\cos y) \cos (k y) d y
$$

We know (see the proof of Lemma 1) that $\{\cos (k y)\}_{k=0}^{\infty}$ is complete in $L_{2}[0, \pi]$. Therefore, it follows immediately from (30) that $g(x)=0$ for almost all $x \in[-1,1]$, as required. Thus, completness is proved.

Finally, we will show that the Chebyshev polynomial system $\left\{t_{k}\right\}_{k=0}^{\infty}$ is orthonor- 
mal over the Chebyshev design $\left\{x_{i}\right\}_{i=1}^{n}$, where $x_{i}=\cos \frac{(2 i-1) \pi}{2 n}$. For positive integers $k \neq l$, due to identities (25) and (24),

$$
\begin{aligned}
\left\langle t_{k}, t_{l}\right\rangle & =\frac{1}{n} \sum_{i=1}^{n} t_{k}\left(x_{i}\right) t_{l}\left(x_{i}\right) \\
& =\frac{2}{n} \sum_{i=1}^{n} \cos \left(k \arccos x_{i}\right) \cos \left(l \arccos x_{i}\right) \\
& =\frac{2}{n} \sum_{i=1}^{n} \cos \left(k \arccos \left(\cos \frac{(2 i-1) \pi}{2 n}\right)\right) \cos \left(l \arccos \left(\cos \frac{(2 i-1) \pi}{2 n}\right)\right) \\
& =\frac{1}{n} \sum_{i=1}^{n}\left(\cos \frac{(2 i-1)(k+l) \pi}{2 n}+\cos \frac{(2 i-1)(k-l) \pi}{2 n}\right) \\
& =\frac{1}{2 n} \frac{\sin (k+l) \pi}{\sin \frac{(k+l) \pi}{2 n}}+\frac{1}{2 n} \frac{\sin (k-l) \pi}{\sin \frac{(k-l) \pi}{2 n}}=0 .
\end{aligned}
$$

For $k=0$ and $l=1,2, \ldots$, and using the identity (24)

$$
\begin{aligned}
\left\langle t_{0}, t_{l}\right\rangle & =\frac{1}{n} \sum_{i=1}^{n} t_{0}\left(x_{i}\right) t_{l}\left(x_{i}\right)=\frac{1}{n} \sum_{i=1}^{n} \sqrt{2} \cos \left(l \arccos x_{i}\right) \\
& =\frac{\sqrt{2}}{n} \sum_{i=1}^{n} \cos \left(l \arccos \left(\cos \frac{(2 i-1) \pi}{2 n}\right)\right) \\
& =\frac{\sqrt{2}}{n} \sum_{i=1}^{n} \cos \frac{(2 i-1) l \pi}{2 n}=\frac{\sqrt{2}}{2 n} \frac{\sin \pi k}{\sin \left(\frac{\pi k}{2 n}\right)}=0
\end{aligned}
$$

The above covers all the cases where $k \neq l$. For positive $k=l$, applying (24) and 
$(26)$

$$
\begin{aligned}
\left\langle t_{k}, t_{k}\right\rangle & =\frac{1}{n} \sum_{i=1}^{n} t_{k}\left(x_{i}\right) t_{k}\left(x_{i}\right) \\
& =\frac{2}{n} \sum_{i=1}^{n} \cos \left(k \arccos \left(\cos \frac{(2 i-1) \pi}{2 n}\right)\right) \cos \left(l \arccos \left(\cos \frac{(2 i-1) \pi}{2 n}\right)\right) \\
& =\frac{2}{n} \sum_{i=1}^{n} \cos ^{2}\left(k \arccos \left(\cos \frac{(2 i-1) \pi}{2 n}\right)\right)=\frac{2}{n} \sum_{i=1}^{n} \cos ^{2}\left(\frac{(2 i-1) k \pi}{2 n}\right) \\
& =\frac{1}{n} \sum_{i=1}^{n}\left(1+\cos \frac{(2 i-1) 2 k \pi}{2 n}\right)=1+\frac{1}{n} \sum_{i=1}^{n} \cos \frac{(2 i-1) 2 k \pi}{2 n} \\
& =1+\frac{1}{2 n} \frac{\sin 2 k \pi}{\sin \left(\frac{k \pi}{2 n}\right)}=1 .
\end{aligned}
$$

And for $k=l=0$,

$$
\left\langle t_{0}, t_{0}\right\rangle=\frac{1}{n} \sum_{i=1}^{n} t_{0}\left(x_{i}\right) t_{0}\left(x_{i}\right)=\frac{1}{n} \sum_{i=1}^{n} 1^{2}=1
$$

Thus, we have shown that the normalized Chebyshev polynomials $t_{k}(x), k=0,1, \ldots$ constitute a complete double-orthonormal system. Lemma 3.3 is proved.

Lemmas 3.1, 3.2, and 3.3 will be now used to construct appropriate projection estimators of an unknown regression function $f$. Assume that $f$ is square integrable (with or without weight) on a finite interval $[a, b]$. In the following, the interval $[a, b]$ will represent either $[0,1]$ or $[-1,1]$, depending on the case.

Case 1. Consider the space $L_{2}([0,1])$ and the uniform design defined by (17). The regression function $f \in L_{2}([0,1])$ can be represented by its cosine-Fourier series

$$
f(x)=\sum_{k=0}^{\infty} d_{k} q_{k}(x)
$$

with Fourier coefficients $d_{k}=\left(f, q_{k}\right)=\int_{0}^{1} f(x) q_{k}(x) d x$. Taking the $N$ th partial sum 
of the series and estimating the first $N$ Fourier coefficients by means of the estimators

$$
\tilde{d}_{k}=\tilde{d}_{k}(\mathbf{Y}):=\left\langle\mathbf{Y}, q_{k}\right\rangle=\frac{1}{n} \sum_{i=1}^{n} Y_{i} q_{k}\left(x_{i}\right), \quad k=0, \ldots, N-1
$$

where $\left\{x_{i}\right\}_{i=1}^{n}$ is the uniform design on $[0,1]$, we arrive at the projection estimator

$$
\tilde{f}_{n, 1}(x)=\sum_{k=0}^{N-1} \tilde{d}_{k} q_{k}(x)
$$

where $N=N_{n}$ is such that, as $n \rightarrow \infty$,

$$
N \rightarrow \infty \text { and } n^{-1} N \rightarrow 0
$$

As shown above, $\tilde{d}_{k}$ is an unbiased estimator of $d_{k}, k=0, \ldots, N-1$. Also, as in the case of kernel estimation, the above assumption on $N$ ensures that the projection estimator is, at least, consistent.

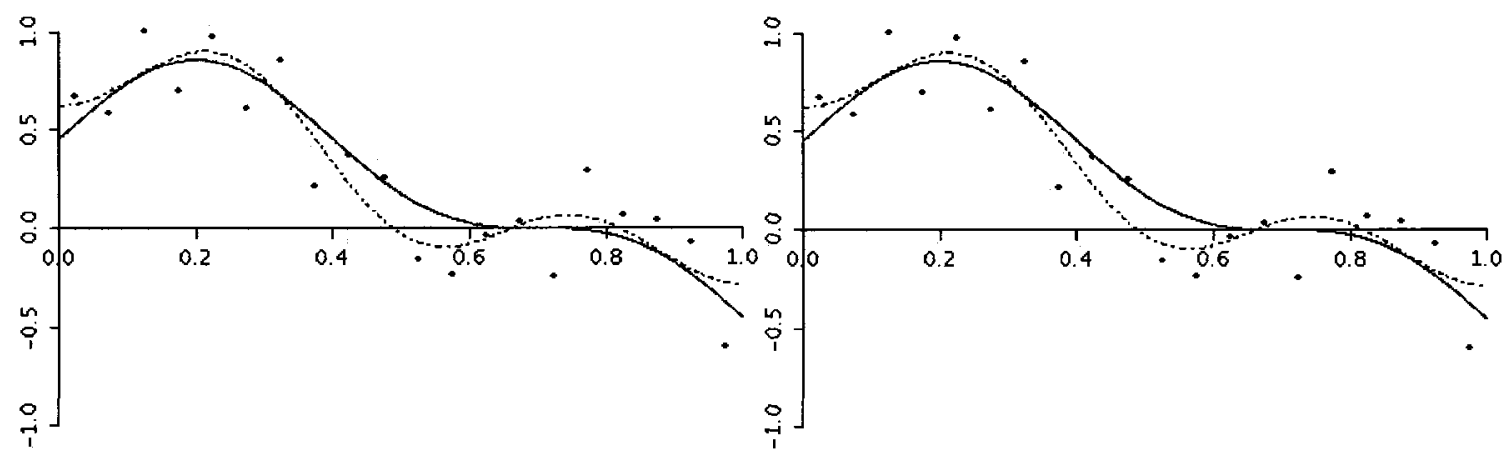
(a) $N_{C V}=5$
(b) $N_{G C V}=5$

Figure 6: The regression estimator $\tilde{f}_{n, 1}(x)=\sum_{k=0}^{N-1} \tilde{d}_{k} q_{k}(x)$ (dotted line) is shown. It is based on $n=20$ data points from $Y_{i}=f\left(x_{i}\right)+\varepsilon_{i}$, where $x_{i}=\frac{2 i-1}{2 n}$. The regression function is given by $f(x)=-0.95 \cos ^{3}(2 \pi x+1.6 \pi)$ (solid line) and the error terms $\varepsilon_{1}, \ldots, \varepsilon_{n}$ are randomly generated from a normal distribution with mean 0 and standard deviation 0.2. The smoothing parameter values, $N_{C V}=5$ and $N_{G C V}=5$, were obtained by the methods of cross-validation and generalized cross-validation, respectively (see Chapter 5 for details). 
Case 2. Consider the space $L_{2}([-1,1])$ and the uniform design $\left\{x_{i}\right\}_{i=-n}^{n}$ defined in (18). The regression function $f \in L_{2}([-1,1])$ can be written in the form of its trigonometric-Fourier series

$$
f(x)=\sum_{k=0}^{\infty} a_{k} h_{k}(x)
$$

where $a_{k}=\left(f, h_{k}\right)=\int_{-1}^{1} f(x) h_{k}(x) d x$. Taking the $N$ th partial sum of the series and estimating the first $N$ Fourier coefficients by their unbiased estimators

$$
\tilde{a}_{k}=\tilde{a}_{k}(\mathbf{Y}):=\left\langle\mathbf{Y}, h_{k}\right\rangle=\frac{2}{2 n+1} \sum_{i=-n}^{n} Y_{i} h_{k}\left(x_{i}\right), \quad k=0, \ldots, N-1
$$

we obtain the projection estimator

$$
\tilde{f}_{n, 2}(x)=\sum_{k=0}^{N-1} \tilde{a}_{k} h_{k}(x)
$$

where $N=N_{n}$ satisfies, as $n \rightarrow \infty$,

$$
N \rightarrow \infty \text { and } \quad n^{-1} N \rightarrow 0
$$




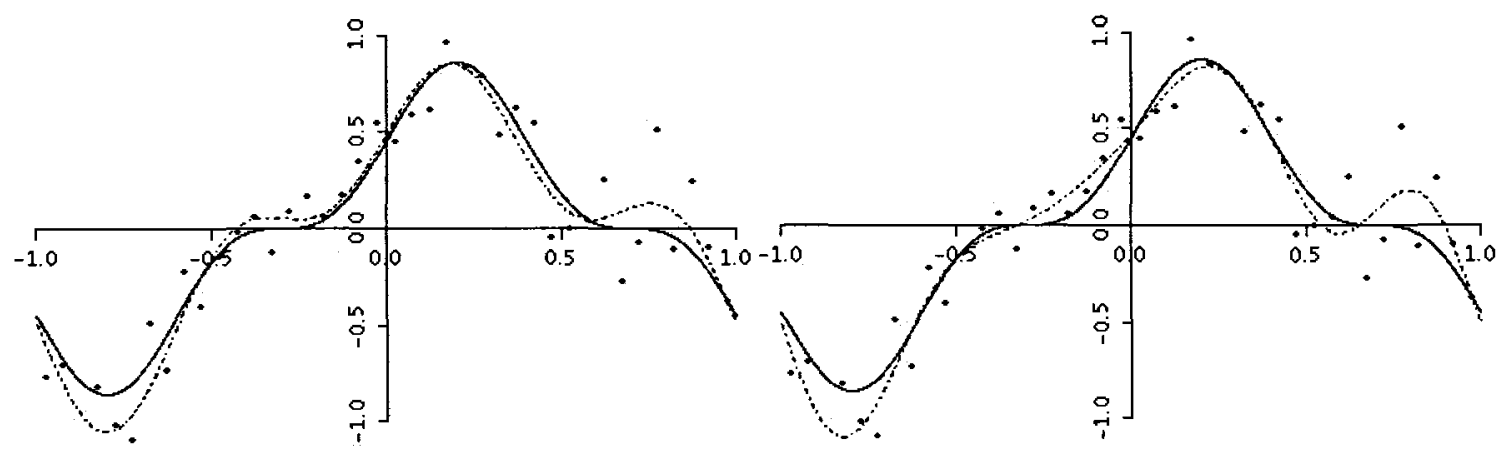

(a) $N_{C V}=7$

(b) $N_{G C V}=9$

Figure 7: The regression estimator $\tilde{f}_{n, 2}(x)=\sum_{k=0}^{N-1} \tilde{a}_{k} h_{k}(x)$ (dotted line) is shown. It is based on $n=40$ data points from $Y_{i}=f\left(x_{i}\right)+\varepsilon_{i}$, where $x_{i}=\frac{2 i}{2 n+1}$. The regression function is given by $f(x)=-0.95 \cos ^{3}(2 \pi x+1.6 \pi)$ (solid line) and the error terms $\varepsilon_{1}, \ldots, \varepsilon_{n}$ are randomly generated from a normal distribution with mean 0 and standard deviation 0.2 . The smoothing parameters $N_{C V}=7$ and $N_{G C V}=9$ were obtained by the methods of cross-validation and generalized crossvalidation, respectively (see Chapter 5 for details).

Case 3. Consider the space $L_{2}([-1,1], \omega)$ with weight function $\omega(x)=1 / \sqrt{1-x^{2}}$ and scalar product (21). Assuming that $f \in L_{2}([-1,1], \omega)$, we can expand $f(x)$ into the Chebyshev-Fourier series

$$
f(x)=\sum_{k=0}^{\infty} b_{k} t_{k}(x)
$$

with Fourier coefficients

$$
b_{k}=\left(f, t_{k}\right)=\frac{1}{\pi} \int_{-1}^{1} \frac{f(x) t_{k}(x)}{\sqrt{1-x^{2}}} d x .
$$

Cutting the summation at a finite point $(N-1)$ and estimating $b_{k}$ by its unbiased estimator

$$
\tilde{b}_{k}:=\left\langle\mathbf{Y}, t_{k}\right\rangle=\frac{1}{n} \sum_{i=1}^{n} Y_{i} t_{k}\left(x_{i}\right), \quad k=0, \ldots, N-1
$$


we obtain a projection estimator of $f(x)$ of the form

$$
\tilde{f}_{n, 3}(x)=\sum_{k=0}^{N-1} \tilde{b}_{k} t_{k}(x)
$$

where, as before, $N=N_{n}$ is such that, as $n \rightarrow \infty$,

$$
N \rightarrow \infty \text { and } n^{-1} N \rightarrow 0
$$

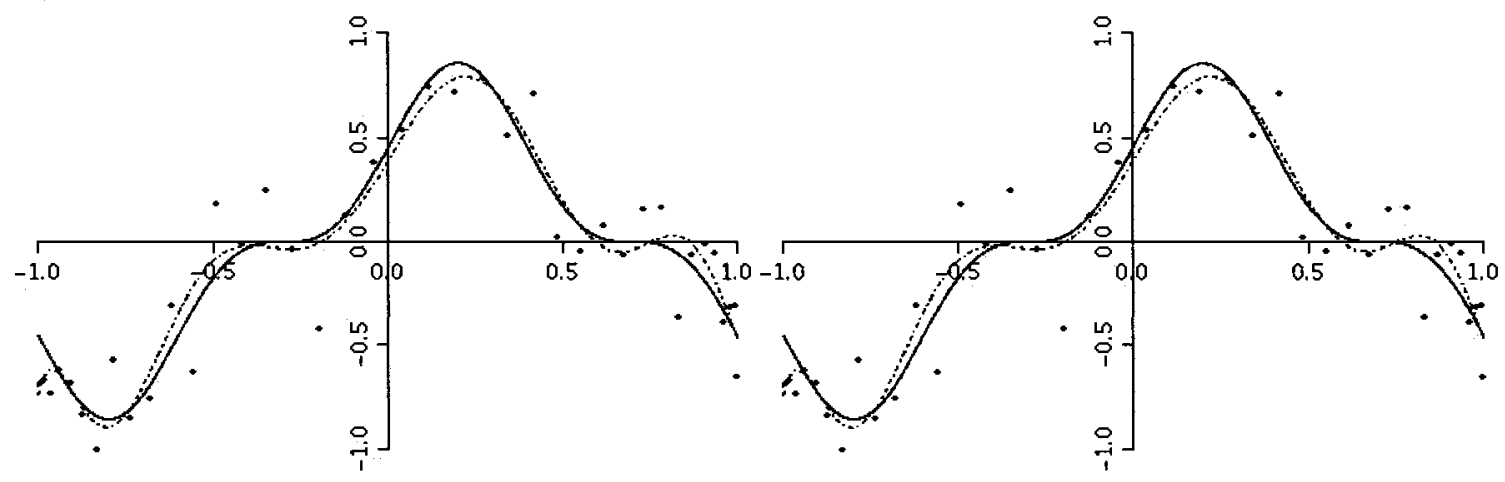
(a) $N_{C V}=10$
(b) $N_{G C V}=10$

Figure 8: The regression estimator $\tilde{f}_{n, 3}(x)=\sum_{k=0}^{N-1} \tilde{b}_{k} t_{k}(x)$ (dotted line) is shown. It is based on $n=40$ data points from $Y_{i}=f\left(x_{i}\right)+\varepsilon_{i}$, where $x_{i}=\frac{(2 i-1) \pi}{2 n}$. The regression function is given by $f(x)=-0.95 \cos ^{3}(2 \pi x+1.6 \pi)$ (solid line) and the error terms $\varepsilon_{1}, \ldots, \varepsilon_{n}$ are randomly generated from a normal distribution with mean 0 and standard deviation 0.2. The smoothing parameters $N_{C V}=10$ and $N_{G C V}=10$ were obtained by the methods of cross-validation and generalized cross-validation, respectively (see Chapter 5 for details).

An important property of the projection estimators constructed above is that they are linear in the vector of observations $\mathbf{Y}=\left(Y_{1}, \ldots, Y_{n}\right)^{\top}$. Indeed, consider the projection estimator $\tilde{f}_{n}(x)=\sum_{k=0}^{N-1} \tilde{c}_{k} \phi_{k}(x)$, where $\left\{\phi_{k}\right\}_{k=1}^{\infty}$ is a complete doubleorthonormal system for a given design $\left\{x_{i}\right\}_{i=1}^{n}$ and where $\tilde{c}_{k}=\left\langle\mathbf{Y}, \phi_{n}\right\rangle$. Changing the 
order of summation, we obtain

$$
\begin{aligned}
\tilde{f}_{n}(x) & =\sum_{k=0}^{N-1} \tilde{c}_{k} \phi_{k}(x)=\sum_{k=0}^{N-1}\left(\frac{1}{n} \sum_{i=1}^{n} Y_{i} \phi_{k}\left(x_{i}\right)\right) \phi_{k}(x)=\frac{1}{n} \sum_{i=1}^{n} Y_{i} \sum_{k=0}^{N-1} \phi_{k}\left(x_{i}\right) \phi_{k}(x) \\
& =\frac{1}{n} \sum_{i=1}^{n} Y_{i} k_{n}\left(x, x_{i}\right)
\end{aligned}
$$

where $k_{n}\left(x, x_{i}\right)=\sum_{k=0}^{N-1} \phi_{k}\left(x_{i}\right) \phi_{k}(x)$.

Figures 6-8 show projection estimators for different designs when the smoothing parameter $N$ is chosen by the methods of cross-validation and generalized crossvalidation. Comparing Figure 7 and Figure 8 shows that near the boundary points $x= \pm 1$ the accuracy of the estimator $\tilde{f}_{n, 3}(x)$ based on the Chebyshev design is higher. The estimator $\tilde{f}_{n, 2}(x)$ based on the uniform design performs less well. Visually, the risk of $\tilde{f}_{n, 2}(x)$ near $x= \pm 1$ is higher relative to the risk computed at the interior points. The Chebyshev design has a larger frequency of observations near the endpoints than in the interior, and can therefore reduce the risk near the boundaries by increasing the amount of information that is available for the estimator. A detailed study of this boundary effect can be found in Artiles (2001), Chapter 3 .

It is worth noting that in some interesting cases, the optimal choice of the parameter $N$ that leads to an asymptotically minimax estimator is nearly inversely proportional to the optimal choice of bandwidth $h$ when the kernel estimator $f_{n}(x)=$ $\sum_{i=1}^{n} n^{-1} K_{h}\left(x-x_{i}\right) Y_{i}$ is used for estimating the regression function. See, for example, Guerre and Tsybakov (1998) and Levit and Stepanova (2004) for this comparison.

\subsection{Relationship to Kernel Regression Estimators}

In the previous section, we have seen that the asymptotic risk of a kernel regression estimator decays to 0 as $n \rightarrow \infty$. Now, let us compare the performance of a kernel 
estimator with that of an orthogonal series estimator. One key difference between these two types of estimators is that a kernel estimator can be applied to any design, while an orthogonal series estimator can be constructed only for a few specific designs, such as the uniform design or the Chebyshev design. Using the argument of Eubank (1999), Section 4.2, we give an example of an instance when a kernel estimator is approximately equal to a damped cosine series estimator.

Let $\mathbf{Y}=\left(Y_{1}, \ldots, Y_{n}\right)^{\top}$ be the response vector in model (1) with observation interval $[-1,1]$ and assume the uniform design $x_{i}=(2 i-1) / 2 n, i=1, \ldots, n$. Now, let $x_{k}$ be a design point that satisfies $x_{k} \in\left[h+(2 n)^{-1}, 1-h-(2 n)^{-1}\right]$. This assumption on $x_{k}$ is made to avoid difficulties at the boundaries. Suppose we wish to estimate the regression function $f$ at the point $x_{k}$ by means of kernel estimator

$$
f_{n}(x)=n^{-1} \sum_{i=1}^{n} K_{h}\left(x-x_{i}\right) Y_{i}
$$

For a kernel $K$ with support on $[-1,1]$, the scaled kernel $K_{h}\left(x_{k}-x_{i}\right)$ is zero if $\left|x_{k}-x_{i}\right|>h^{-1}$, that is, if $i<k-n h$ and $i>k+n h$. Because $i$ is an integer, we have $K_{h}\left(x_{k}-x_{i}\right)=0$ if $i<k-[n h]$ and $i>k+[n h]$, where $[n h]$ denotes the integer part of $n h$. In this case, the vector of scaled kernels at the design point will be

$$
\begin{aligned}
\mathbf{K}_{n}= & \left(K_{h}\left(x_{1}-x_{k}\right), \ldots, K_{h}\left(x_{n}-x_{k}\right)\right)^{\top} \\
= & \left(0, \ldots, 0, K_{h}\left(-\frac{[n h]}{n h}\right), K_{h}\left(-\frac{[n h]-1}{n h}\right), \ldots\right. \\
& \left.\ldots, K_{h}\left(\frac{[n h]-1}{n h}\right), K_{h}\left(\frac{[n h]}{n h}\right), 0, \ldots, 0\right)^{\top} .
\end{aligned}
$$

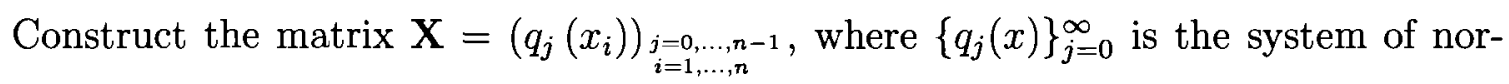


malized cosine polynomials defined by

$$
q_{j}(x)= \begin{cases}1 & , j=0, \\ \sqrt{2} \cos (j \pi x) & , j=1,2, \ldots\end{cases}
$$

As shown in Chapter 3, the cosine sequence is orthonormal with respect to the uniform design. Therefore, $\mathbf{X}^{\top} \mathbf{X}=\mathbf{X X}^{\top}=n \mathbf{I}$. Using this fact, we can express the kernel estimator $f_{n}$ evaluated at the $k$ th design point, $f_{n}\left(x_{k}\right)$, as the transpose of a vector $\mathbf{m}_{n}=n^{-1} \mathbf{X}^{\top} \mathbf{K}_{n}$ times a vector $\mathbf{b}=n^{-1} \mathbf{X}^{\top} \mathbf{Y}=\left(n^{-1} \sum_{i=1}^{n} Y_{i} q_{j}\left(x_{i}\right)\right)_{j=0}^{n-1}$ of the sample cosine Fourier coefficients defined in Section 3.2:

$$
\begin{aligned}
f_{n}\left(x_{k}\right) & =n^{-1} \mathbf{K}_{n}^{\top} \mathbf{Y}=n^{-1} \mathbf{K}_{n}^{\top}\left(n^{-1} \mathbf{X} \mathbf{X}^{\top}\right) \mathbf{Y} \\
& =n^{-1} \mathbf{K}_{n}^{\top} \mathbf{X} n^{-1} \mathbf{X}^{\top} \mathbf{Y}=\mathbf{m}_{n}^{\top} \mathbf{b} .
\end{aligned}
$$

In particular, the $j$ th column of $\mathbf{m}_{n}, j=1, \ldots, n-1$ is

$$
\begin{aligned}
\left(\mathbf{m}_{n}\right)_{j} & =(n h)^{-1} \sum_{i=1}^{n} \sqrt{2} \cos \left(j \pi x_{i}\right) K\left(\frac{x_{k}-x_{i}}{h}\right) \\
& =(n h)^{-1} \sum_{i=1}^{n} \sqrt{2} \cos \left(j \pi\left(x_{i}-x_{k}\right)+j \pi x_{k}\right) K\left(\frac{x_{k}-x_{i}}{h}\right) \\
& =(n h)^{-1} \sum_{l=-[n h]}^{[n h]} \sqrt{2} \cos \left(-j \pi \frac{l}{n}+j \pi x_{k}\right) K\left(\frac{l}{n h}\right) .
\end{aligned}
$$

Using the trigonometric identity $\cos (x-y)=\cos x \cos y+\sin x \sin y$, we can obtain for $j=1, \ldots, n$ 


$$
\begin{aligned}
\left(\mathbf{m}_{n}\right)_{j} & =(n h)^{-1} \sum_{l=-[n h]}^{[n h]} \sqrt{2} \cos \left(-j \pi \frac{l}{n}+j \pi x_{k}\right) K\left(\frac{l}{n h}\right) \\
& =\sqrt{2}(n h)^{-1} \sum_{l=-[n h]}^{[n h]} K\left(\frac{l}{n h}\right)\left(\cos \left(-j \pi \frac{l}{n}\right) \cos \left(j \pi x_{k}\right)+\sin \left(-j \pi \frac{l}{n}\right) \sin \left(j \pi x_{k}\right)\right) \\
& =\sqrt{2}(n h)^{-1} \sum_{l=-[n h]}^{[n h]} K\left(\frac{l}{n h}\right) \cos \left(j \pi \frac{l}{n}\right) \cos \left(j \pi x_{k}\right) \\
& =\sqrt{2} \cos \left(j \pi x_{k}\right) n^{-1} \sum_{l=-[n h]}^{[n h]} h^{-1} K\left(\frac{l}{n h}\right) \cos \left(j \pi \frac{l}{n}\right) \\
& =\sqrt{2} \cos \left(j \pi x_{k}\right) m_{j n},
\end{aligned}
$$

where $m_{j n}=n^{-1} \sum_{l=-[n h]}^{[n h]} h^{-1} K\left(\frac{l}{n h}\right) \cos \left(j \pi \frac{l}{n}\right)$. For $j=0$,

$$
\begin{aligned}
\left(\mathbf{m}_{n}\right)_{0} & =(n h)^{-1} \sum_{l=-[n h]}^{[n h]} \sqrt{2} K\left(\frac{l}{n h}\right) \\
& =\sqrt{2} m_{0 n},
\end{aligned}
$$

where $m_{0 n}=n^{-1} \sum_{l=-[n h]}^{[n h]} h^{-1} K\left(\frac{l}{n h}\right)$. Thus, the kernel regression estimator $f_{n}(x)$ can be written as a damped cosine series estimator as follows

$$
\begin{aligned}
f_{n}(x) & =m_{0 n} b_{0 n}+\sum_{j=1}^{n-1} m_{j n} b_{j n} \sqrt{2} \cos \left(j \pi x_{k}\right) \\
& =\sum_{j=0}^{n-1} m_{j n} b_{j n} q_{j}\left(x_{k}\right) .
\end{aligned}
$$

Here $b_{j n}$ is the sample $j$ th cosine Fourier coefficient and the damping factor $m_{j n}$ is approximately the $j$ th cosine Fourier transform of the kernel function $K$ evaluated at the point $j \pi h$. The behaviour of the Fourier transforms of standard kernels with 
finite support is well-known, and so the damping factors are easy to work with.

For example, the uniform and the Epanechnikov kernels can be used in this case since they have support on $[-1,1]$. Their cosine Fourier transforms are shown in Figure 9. Notice that they are symmetric about 0 and attain their maximum at $x=0$.

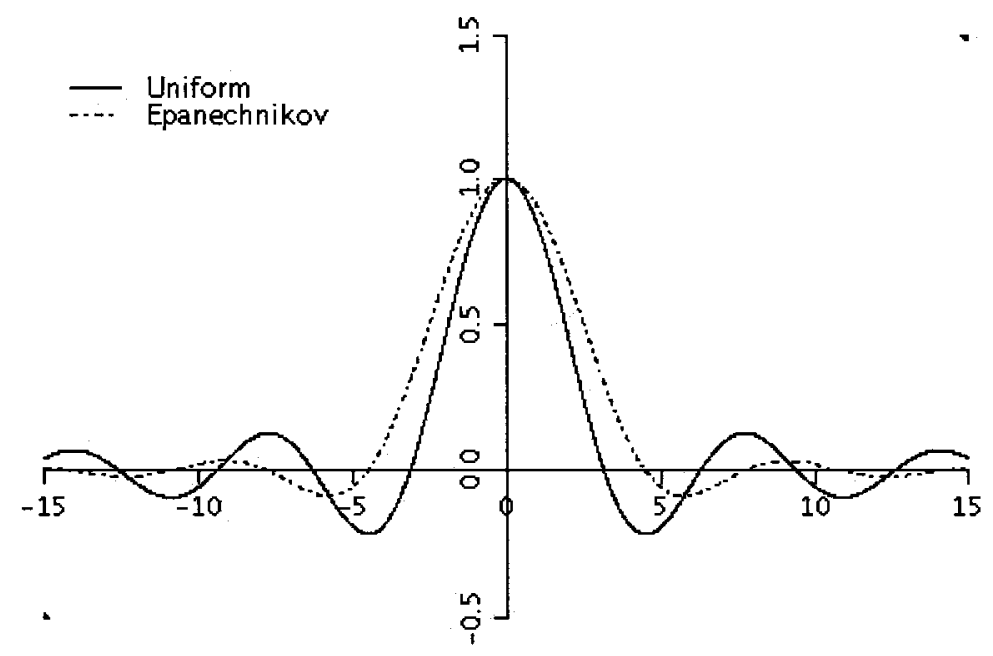

Figure 9: Cosine Fourier transforms of the uniform and Epanechnikov kernels (kernels with support on $[-1,1]$.)

This means that the damping factor $m_{j n}$ tends to be lower for larger values of $|j|$, that is, this damping factor tends to give more weight to the lower frequency Fourier coefficients (the leading terms of the estimator) than to the higher frequency Fourier coefficients of the orthogonal series estimator. Although, this argument makes many assumptions, it is very useful as it links the topics of kernel regression estimation with orthogonal series estimation. 


\section{Spline Smoothing}

\subsection{Definition and Basic Properties of Spline Functions}

Smoothing spline estimators of the regression curve are widely used in the areas of data fitting and geometric modeling. Historically, splines were primarily seen as a topic in Numerical Analysis, and their approximation properties were first investigated in detail by Schoenberg (1946). They first appeared in the context of smoothing as a method of "graduating" data (see Whittaker, 1923), that is, as a method of removing fluctuations due to random errors in raw data by achieving a balance between a measure of fit and a measure of smoothness of the estimated function. It was not until relatively recently that splines were applied to the problem of nonparametric regression by Wahba (1978).

Definition 4.1. Let $\left\{x_{1}, \ldots, x_{n}\right\}$ be a set of $n$ distinct design points satisfying

$$
a<x_{1}<\ldots<x_{n}<b
$$

and let $x_{0}=a, x_{n+1}=b$. A spline of degree $r$ (order $\left.r+1\right)$ on the interval $[a, b]$ with respect to $\left\{x_{0}, x_{1}, \ldots, x_{n}, x_{n+1}\right\}$ is an $(r-1)$-times continuously differentiable function that agrees with a polynomial of degree at most $r$ on each grid interval $\left[x_{i}, x_{i+1}\right]$ for $i=0, \ldots, n$.

Let $S^{r}\left(x_{0}, \ldots, x_{n+1}\right)$ denote the space of splines of degree $r$ on the interval $[a, b]$ with respect to the knots $x_{1}, \ldots, x_{n}$ with $x_{0}=a, x_{n+1}=b$. It follows from this definition that $s \in S^{r}\left(x_{0}, \ldots, x_{n+1}\right)$ is a piecewise polynomial of degree $r$ on any subinterval $\left[x_{i}, x_{i+1}\right]$, which has $r-1$ continuous derivatives and has a discontinuous $r$ th derivative with jumps at the design points $x_{1}, \ldots, x_{n}$.

Figure 10 shows a spline $s$ of degree 3, also known as a cubic spline, with re- 


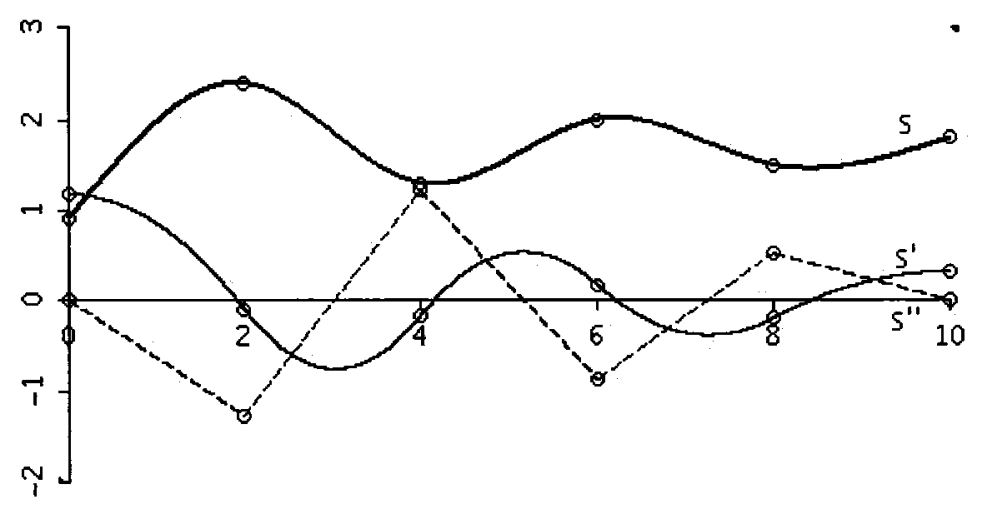

Figure 10: A cubic spline $s$ (thick solid line), its first derivative $s^{\prime}$ ( thin solid line) and its second derivative $s^{\prime \prime}$ (dashed line) with respect to $\{0,2,4,6,8,10\}$.

spect to $\{0,2,4,6,8,10\}$. The first derivative of the cubic spline is a spline of degree 2 (thin solid line). The second derivative of the cubic spline is a spline of degree 1. As suggested in the definition, the cubic spline is twice continuously differentiable. Moreover, computing the third derivative of $s$ reveals jumps at the design points. Figure 10 was obtained by fitting an interpolating spline to the data points $(0,0.9),(2,2.4),(4,1.3),(6,2),(8,1.5),(10,1.8)$ using Matlab. The resulting equations of the piecewise third degree polynomials that form the cubic spline $s$ were plotted with S-Plus. The equations were differentiated once to obtain and plot the first derivative $s^{\prime}$ and then were differentiated again to obtain the second derivative $s^{\prime \prime}$.

Natural splines form a useful subspace of the space of splines. Natural splines are obtained by solving the optimization problem of finding a nonparametric regression estimator that balances smoothness with fit to the data.

Definition 4.2. A natural spline of degree $r=2 m-1$ (order $2 m$ ) on the interval $[a, b]$ with knots $x_{1}, \ldots, x_{n}$, where $a=x_{0}<x_{1}<\ldots<x_{n}<x_{n+1}=b$, is a spline function $s \in S^{r}\left(x_{0}, \ldots, x_{n+1}\right)$ such that $s(x)$ agrees with a polynomial of degree at most $m$ outside of the interval $\left[x_{1}, x_{n}\right]$.

Denote the space of natural splines of degree $2 m-1$ on the interval $[a, b]$ with 
knots $x_{1}, \ldots, x_{k}$, and $x_{0}=a, x_{n+1}=b$ by $N S^{2 m-1}\left(x_{0}, \ldots, x_{n+1}\right)$. Because the natural spline space $N S^{2 m-1}\left(x_{0}, \ldots, x_{n+1}\right)$ is obtained by imposing $2 m$ linear restrictions on the spline space $S^{2 m-1}\left(x_{0}, \ldots, x_{n+1}\right)$, namely ensuring that every element of the space of natural splines is also a polynomial of degree at most $m$ outside of the interval $\left[x_{1}, x_{n}\right]$, its dimension is

$\operatorname{dim}\left\{N S^{2 m-1}\left(x_{0}, \ldots, x_{n+1}\right)\right\}=\operatorname{dim}\left\{S^{2 m-1}\left(x_{0}, \ldots, x_{n+1}\right)\right\}-2 m=(n+2 m)-2 m=n$

That is, the dimension of the natural spline space does not depend on the degree, but on the number of design knots, $n$. As we shall see, fitting smoothing splines will require the selection of a set of convenient basis functions for the space of natural splines. A popular basis for $N S^{2 m-1}\left(x_{0}, \ldots, x_{n+1}\right)$ is the $B$-spline basis. For some positive integer $r$, the $\mathbf{B}$-spline basis is a set

$$
\mathcal{B}_{r}=\left\{b_{1}^{r}(x), \ldots, b_{n}^{r}(x)\right\}
$$

where $b_{i}^{r}$ are $B$-splines of degree $r, i=1, \ldots, n$ defined in terms of so-called "divided differences". The functions $b_{i}^{r}(x)$ are defined explicitly in Theorem 17.1 of Greville (1969). Statistical software such as S-Plus offers functions that compute B-splines for use in smoothing. However, in this thesis, we do not touch on theoretical aspects that would require using the explicit form of the B-spline basis $\mathcal{B}_{r}$.

Theorem 4.2 (Greville, 1969). The set of B-splines (31) with $r=2 m-1$ forms a basis of the natural spline space $N S^{2 m-1}\left(x_{0}, \ldots, x_{n+1}\right)$.

Theorem 4.2 implies that any element $s$ of the space $N S^{2 m-1}\left(x_{0}, \ldots, x_{n+1}\right)$ can 
be written in the form,

$$
s(x)=\sum_{i=1}^{n} c_{i} b_{i}^{2 m-1}(x),
$$

where $c_{1}, \ldots, c_{n}$ are constants called De Boor points of $s(x)$.

From now on, let us assume, without loss of generality, that $a=0$ and $b=1$. De Boor (1963) was the first to show that a natural spline that agrees with the data at the design points is the unique minimizer, among all functions $g \in W_{2}^{m}[0,1]$, of a measure of curvature over the entire curve

$$
J_{m}(g)=\int_{0}^{1}\left(g^{(m)}(x)\right)^{2} d x
$$

This fact will be used below to show that a natural spline is an optimal estimator in the sense that it can be used to balance, based on the value of a smoothing parameter, good fit to the data with a measure of smoothness.

Lemma 4.1 (De Boor, 1963). Let $q_{1}, \ldots, q_{n}$ be a basis for $N S^{2 m-1}\left(x_{0}, \ldots, x_{n+1}\right)$ and let $\boldsymbol{a}=\left(a_{1}, \ldots, a_{n}\right)^{\top}$ be a vector of constants. Put $\boldsymbol{Q}=\left(q_{j}\left(x_{i}\right)\right)_{n \times n}$ and $\boldsymbol{g}=$ $\left(g\left(x_{1}\right), \ldots, g\left(x_{n}\right)\right)^{\top}$, for some function $g$. Then if $n \geq m$, the unique minimizer of $J_{m}(g)=\int_{0}^{1}\left(g^{(m)}(x)\right)^{2} d x$ over all $g \in W_{2}^{m}[0,1]$ that satisfy $\boldsymbol{g}=\boldsymbol{a}$ is

$$
s=\sum_{j=1}^{n} d_{j} q_{j}
$$

where $\boldsymbol{d}=\left(d_{1}, \ldots, d_{n}\right)^{\top}$ is the unique solution to $\boldsymbol{Q d}=\boldsymbol{a}$. In particular, the matrix $\boldsymbol{Q}$ has full rank $n$. 


\subsection{Spline Smoothing Estimators}

The aim of the problem of nonparametric regression is to select, from among a class of estimators, an estimator that is as "close" as possible to the true regression function, and that has certain desirable properties. Some criteria for measuring "closeness" were discussed in the introduction to this thesis. In choosing a performance criterion, one must consider the important properties desired from a "good" estimator. First, we would like the estimator to minimize some measure of distance between itself and the true regression function, and second, we would like the estimator to be reasonably smooth. The first criterion suggests using a measure of fit, such as the average regression sum of squares, which can be calculated from the data. The average regression sum of squares for some function $g$ is a local performance criterion for the deviation of $g$ from the response values at the design points:

$$
n^{-1} R S S(g)=n^{-1} \sum_{i=1}^{n}\left(Y_{i}-g\left(x_{i}\right)\right)^{2}
$$

In fact, the average regression sum of squares is an estimator for the prediction risk (its properties will be discussed in detail in Chapter 5). A small value of (33) implies that values of $g$ and the observed values of $Y$ are close at the design points. However, agreement at the design points does not imply agreement elsewhere.

Theorem 4.2 (see, for example, Deuflhart and Hoffman, 2000). For $n+1$ distinct design points $x_{0}, \ldots, x_{n}$ and corresponding observations $g\left(x_{i}\right), i=0, \ldots, n$ there exists a unique interpolating polynomial $P$ from the space $\mathcal{P}_{n}\left[x_{0}, x_{n}\right]$ of all polynomials of order $n$ such that $P\left(x_{i}\right)=g\left(x_{i}\right)$ for $i=0, \ldots, n$.

It follows form Theorem 4.2 that one can always find at least one estimator that interpolates the data, that is, one can always interpolate the data by fitting a polynomial of sufficiently high degree. Interpolating the data minimizes the average regression 
sum of squares despite the fact that the polynomial may oscillate wildly between the design points. Because such unreasonable oscillation between the design points is not a desirable property for most estimators, we must impose a restriction on oscillations of the estimator. For $g \in W_{2}^{m}[0,1]$, we can use (32) as a measure of curvature of $g$. Until the work of Reinsch (1967), splines had been used for data interpolation, that is, for minimization of (33) only. But, as demonstrated by Theorem 4.2, this can result in an estimator with large oscillations between the nodes. Reinsch (1967) proposed to use spline smoothing, which is a compromise between strict data interpolation and data smoothing, by minimizing for some $\lambda>0$ the quantity

$$
I_{m}(g)=n^{-1} \sum_{i=1}^{n}\left(Y_{i}-g\left(x_{i}\right)\right)^{2}+\lambda \int_{0}^{1}\left(g^{(m)}(x)\right)^{2} d x
$$

obtained by adding (32) and (33). The parameter $\lambda$ governs the tradeoff between smoothness and goodness of fit. For this reason, $\lambda$ is often called the smoothing parameter. For given values of $m$ and $\lambda$, the aim is to choose an optimal estimator that minimizes criterion $(34)$ over $W_{2}^{m}[0,1]$. Such an optimal estimator achieves a balance between good fit at the design points and reasonable smoothness as discussed earlier. The smoothing parameter $\lambda$ dictates the emphasis that is put on smoothness over fit. A large $\lambda$ causes the optimal estimator to emphasize smoothness, while a small $\lambda$ leads to more emphasis on goodness-of-fit at the design points. In the limit, choosing $\lambda=0$ results in functions that interpolate the data (for example, by fitting a polynomial of sufficiently high degree to the data), since this sets (33) to zero. Choosing $\lambda=\infty$ produces polynomial estimators of degree $m-1$, since their $m$ th derivative is zero, and thus (32) is minimized. 
Now, consider the regression model

$$
Y_{i}=f\left(x_{i}\right)+\varepsilon_{i}, \quad i=1, \ldots, n,
$$

where $\varepsilon_{i}$ are independent $N\left(0, \sigma^{2}\right)$ normal random variables, $x_{i}$ are design points on the interval $[0,1]$, and $f$ is an unknown regression function that belongs to $W_{2}^{m}[0,1]$. To simplify this setup, assume further that the design points are distinct and that $0<x_{1}<\ldots<x_{n}<1$. Put $x_{0}=0$ and $x_{n+1}=1$ and assume, for now, that a value of $\lambda$ is given. Later, we will generalize these assumptions to the case of non-distinct design points and the case of distinct error term variances (heteroskedastic data). The choice of smoothing parameter $\lambda$ will be discussed in Chapter 5 , and an argument for choosing $m=3$ will be given at the end of this chapter.

The following theorem presents spline smoothing estimators as the solution to an optimization problem for balancing agreement at the design points with smoothness. It is shown that, for a given smoothing parameter $\lambda$, (34) is minimized when we estimate the regression function $f$ by means of a natural spline of degree $2 m-1$ that agrees with the data at the design points.

Theorem 4.3 (Reinsch, 1967). Let $q_{1}, \ldots, q_{n}$ be a basis for the set of natural splines of degree $2 m-1$ with knots $x_{1}, \ldots, x_{n}$ and define the $n \times n$ matrix $\boldsymbol{Q}=\left(q_{j}\left(x_{i}\right)\right)_{n \times n}$. Then, if $n \geq m$ the unique minimizer of (34) is

$$
s=\sum_{j=1}^{n} d_{\lambda j} q_{j}
$$

where $\boldsymbol{d}_{\lambda}=\left(d_{\lambda 1}, \ldots, d_{\lambda n}\right)^{\top}$ is the unique solution of the system

$$
\left(\mathbf{Q}^{\top} \mathbf{Q}+n \lambda \Omega\right) \mathbf{d}=\mathbf{Q}^{\top} \mathbf{Y}
$$


with vector $\boldsymbol{Y}=\left(Y_{1}, \ldots, Y_{n}\right)^{\top}$ and matrix

$$
\boldsymbol{\Omega}=\left(\int_{0}^{1} q_{i}^{(m)}(x) q_{j}^{(m)}(x) d x\right)_{n \times n}
$$

A more straightforward proof than that of Reinsch (1967) is outlined in Eubank (1999), Section 5.2. Let us give a detailed proof of this result.

Proof. We will show that the estimator that minimizes criterion (34) is, in fact, the natural spline (36) whose coefficients are solutions of (37). Let us examine both terms of (34). The first term is minimized by choosing $g$ such that $g\left(x_{i}\right)=Y_{i}$ for $i=1, \ldots, n$. Assuming that $n \geq m$, due to Lemma 4.1 the unique minimizer of $J_{m}(g)=\int_{0}^{1}\left(g^{(m)}(x)\right)^{2} d x$ over all $g \in W_{2}^{m}[0,1]$ that satisfies $g\left(x_{i}\right)=Y_{i}, i=1, \ldots, n$ is $s=\sum_{j=1}^{n} d_{j} q_{j}$, where $\mathbf{d}=\left(d_{1}, \ldots, d_{n}\right)^{\top}$ is the unique solution to $\mathbf{Q d}=\mathbf{Y}$. That is, if we minimize (34) over all functions of the form $s=\sum_{j=1}^{n} d_{j} q_{j}$, we ensure that interpolation becomes possible (although not always necessary). Thus, minimizing (34) is reduced to minimizing

$$
\begin{aligned}
S(\mathbf{d}) & =\frac{1}{n}(\mathbf{Y}-\mathbf{Q d})^{\top}(\mathbf{Y}-\mathbf{Q d})+\lambda \mathbf{d}^{\top} \mathbf{\Omega} \mathbf{d} \\
& =\frac{1}{n} \mathbf{Y}^{\top} \mathbf{Y}-\frac{2}{n} \mathbf{d}^{\top} \mathbf{Q}^{\top} \mathbf{Y}+\frac{1}{n} \mathbf{d}^{\top} \mathbf{Q}^{\top} \mathbf{Q} \mathbf{d}+\lambda \mathbf{d}^{\top} \boldsymbol{\Omega} \mathbf{d}
\end{aligned}
$$

Recall that for two vectors $\mathbf{a}=\left(a_{1}, \ldots, a_{n}\right)^{\top}$ and $\mathbf{b}=\left(b_{1}, \ldots, b_{n}\right)^{\top}$ and a symmetric $n \times n$ matrix $\mathbf{A}$, we have

$$
\frac{d}{d \mathbf{b}}\left(\mathbf{a}^{\top} \mathbf{b}\right)=\frac{d}{d \mathbf{b}}\left(\mathbf{b}^{\top} \mathbf{a}\right)=\mathbf{a}
$$

and

$$
\frac{d}{d \mathbf{b}}\left(\mathbf{b}^{\top} \mathbf{A b}\right)=2 \mathbf{A b}
$$


where $\frac{d}{d \mathbf{b}}=\left(\frac{d}{d b_{1}}, \ldots, \frac{d}{d b_{n}}\right)^{\top}$. The proofs of (38) and (39) are given in the Appendix. Therefore,

$$
\frac{d S(\mathbf{d})}{d \mathbf{d}}=-\frac{2}{n} \mathbf{Q}^{\top} \mathbf{Y}+\frac{2}{n} \mathbf{Q}^{\top} \mathbf{Q} \mathbf{d}+2 \lambda \Omega \mathbf{d}
$$

Setting

$$
\frac{d S(\mathbf{d})}{d \mathbf{d}}=0
$$

gives the equation

$$
\mathbf{Q}^{\top} \mathbf{Y}=\mathbf{Q}^{\top} \mathbf{Q} \mathbf{d}+n \lambda \boldsymbol{\Omega} \mathbf{d}
$$

or equivalently,

$$
\left(\mathbf{Q}^{\top} \mathbf{Q}+n \lambda \Omega\right) \mathbf{d}=\mathbf{Q}^{\top} \mathbf{Y}
$$

It remains to show that system (37) has a unique solution. According to Lemma 4.1, the matrix $\mathbf{Q}$ is of full rank. Next, for all nonzero $\mathbf{d} \in \mathbb{R}^{n}$,

$$
\begin{aligned}
\mathbf{d}^{\top} \boldsymbol{\Omega d} & =\sum_{i=1}^{n} \sum_{j=1}^{n} d_{i} d_{j} \Omega_{i j} \\
& =\sum_{i=1}^{n} \sum_{j=1}^{n} d_{i} d_{j} \int_{0}^{1} q_{i}^{(m)}(x) q_{j}^{(m)}(x) d x \\
& =\int_{0}^{1} \sum_{i=1}^{n} d_{i} q_{i}^{(m)}(x) \sum_{j=1}^{n} d_{j} q_{j}^{(m)}(x) d x \\
& =\int_{0}^{1}\left(h^{(m)}(x)\right)^{2} d x \geq 0,
\end{aligned}
$$

where $h^{(m)}(x)=\sum_{i=1}^{n} d_{i} q_{i}^{(m)}(x)$. That is, $\Omega$ is positive semi-definite, and hence 
$\mathbf{Q}^{\top} \mathbf{Q}+n \lambda \boldsymbol{\Omega}$ is positive definite. Therefore, the solution is unique. Theorem 4.3 is proved.

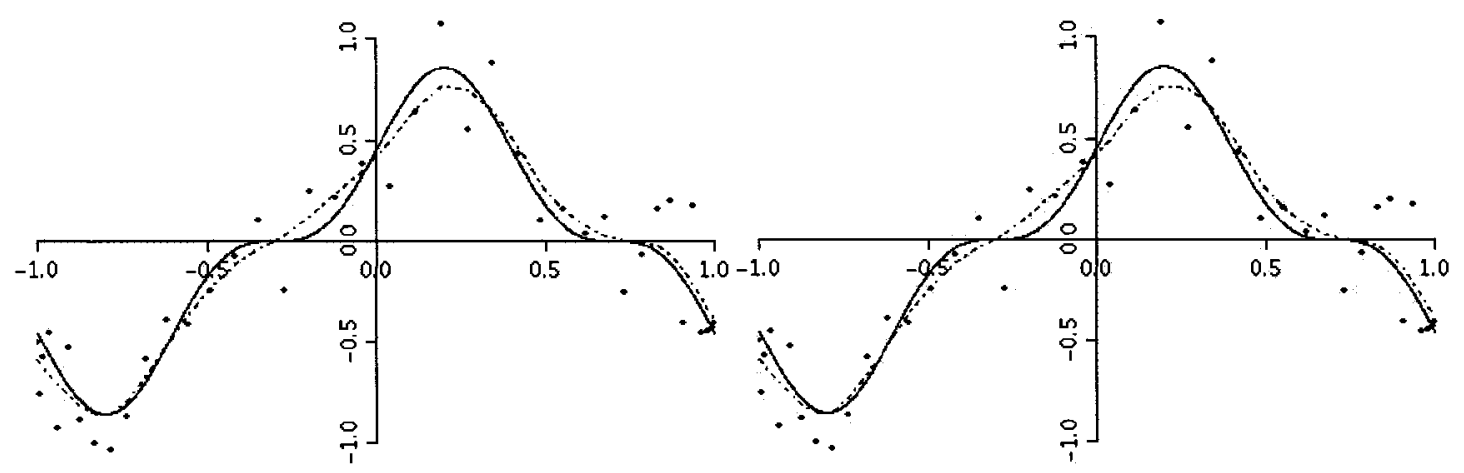

(a) $\lambda_{C V}=0.000099595$

(b) $\lambda_{G C V}=0.0001048302$

Figure 11: A cubic smoothing spline (dotted line) fit to $n=40$ data points from $Y_{i}=$ $f\left(x_{i}\right)+\varepsilon_{i}$, where $\left\{x_{i}\right\}_{i=1}^{n}$ follows the Chebyshev design. The regression function is $f(x)=$ $-0.95 \cos ^{3}(2 \pi x+1.6 \pi)$ (solid line) and the error terms $\varepsilon_{1}, \ldots, \varepsilon_{n}$ are randomly generated from a normal distribution with mean 0 and standard deviation 0.2. The spline smoother was produced by the function smooth.spline() in S-Plus.

So far, we have relied on the assumption that the design knots are distinct. However, many data sets have replications, that is, they have more than one observation corresponding to a single design point. For example, a data set of drop out rates versus crime rates in different cities may have replications if multiple cities have the same drop out rate (but not necessarily the same crime rates). This is equivalent to having overlapping, or non-distinct design points. In such cases, the statistician cannot simply choose to use only one of the replicate observations for a design point without some loss of information.

Example (Liver Function Test): Consider the example of a data set with replications, which uses the score from a liver function test as a predictor of survival times of liver patients. A liver function test is a clinical biochemistry laboratory blood test that evaluates the state of a subject's liver. This test is a tool that can provide early 
detection of liver diseases that are difficult to detect from their mild initial symptoms. We are interested in answering the question of how the score of the liver function test is related to the survival time of a liver patient. This is a typical instance of a case where parametric regression is not appropriate, since it would require the knowledge of the form of the regression function up to a finite number of parameters, which is exactly what we must find.

\begin{tabular}{|c|c|c|c|c|}
\hline Observation & $\begin{array}{c}\text { Liver Function } \\
\text { Test Score } \\
i\end{array}$ & $\begin{array}{c}\text { Survival Time } \\
X_{i}\end{array}$ & $\begin{array}{c}\text { Average } \\
\text { Survival Time } \\
\bar{Y}_{i}\end{array}$ & $\begin{array}{c}\text { Number of } \\
\text { Observations } \\
n_{i}\end{array}$ \\
\hline 1 & 2.1 & 70 & 70 & 1 \\
\hline 2 & 2.4 & 203,217 & 210 & 2 \\
\hline 3 & 0.74 & 71 & 71 & 1 \\
\hline 4 & 2.85 & $153,120,109$ & 127.333 & 3 \\
\hline \multicolumn{5}{|c|}{$\vdots$} \\
\hline 46 & 3.95 & 830,330 & 580 & 2 \\
\hline 47 & 6.4 & 483 & 483 & 1 \\
\hline
\end{tabular}

Table 3: Liver Function Test Data.

Criterion (34) must be generalized to work with non-distinct design knots. We will see that this criterion is just a weighted version of (34). Then, we will find an estimator that minimizes this weighted criterion, thus providing a solution to Exercise 5.9.4 in Eubank (1999).

To generalize the smoothing criterion (34), rewrite model (35) with $n$ distinct design points as

$$
Y_{i j}=f\left(x_{i j}\right)+\varepsilon_{i j}, \quad i=1, \ldots, r, \quad j=1, \ldots, n_{i}
$$

where $\sum_{i=1}^{r} n_{i}=n$. Recall that the first part of the spline criterion (34) for model 


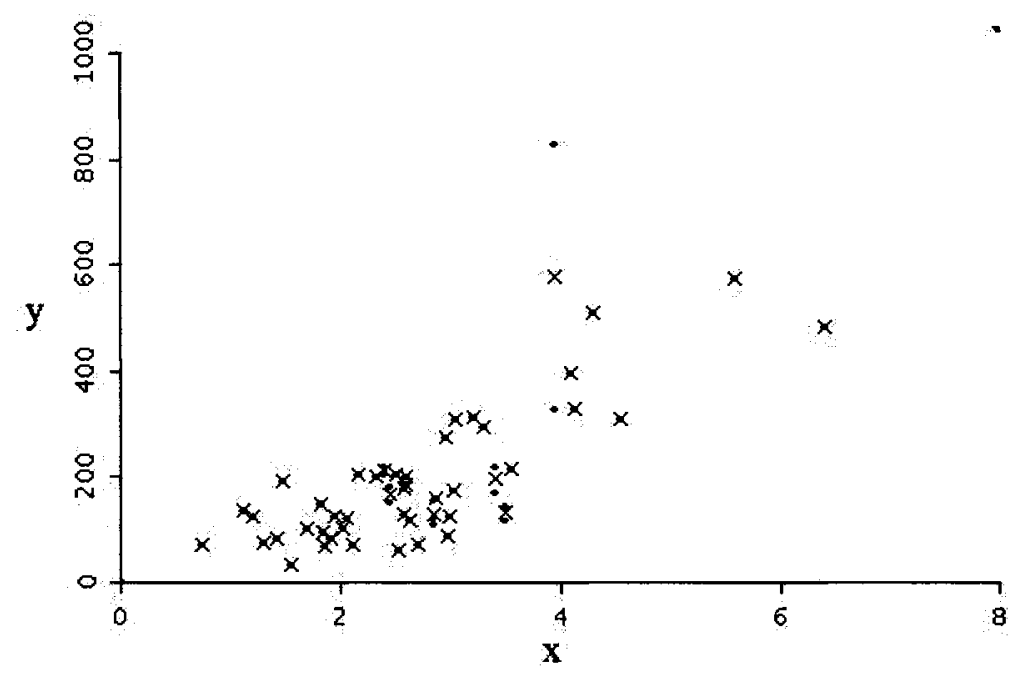

Figure 12: Liver function test scores $\left(X_{i}\right)$ versus survival times $\left(Y_{i}\right)$ of liver patients. The data set was obtained from the paper by Shih and Weisberg (1986). The data has several replications (more than one response value for a single value $X_{i}$ ). For the purpose of spline smoothing, the replicate response were averaged for every level $X_{i}$.

(35) with distinct design points is the average regression sum of squares

$$
n^{-1} R S S(g)=n^{-1} \sum_{i=1}^{r}\left(Y_{i}-g\left(x_{i}\right)\right)^{2} .
$$

Under model (40) this becomes

$$
n^{-1} R S S(g)=n^{-1} \sum_{i=1}^{r} \sum_{j=1}^{n_{i}}\left(Y_{i j}-g\left(x_{i j}\right)\right)^{2}
$$

This statistic measures the deviation of each observation from the estimate $g$ of $f$. Now, assume that not all design points are distinct, that is, assume that $x_{i j}=x_{i}$ for $i=1, \ldots, r$ and $j=1, \ldots, n_{i}$. The regression sum of squares (assuming all design points to be distinct) is also known as the average lack-of-fit sum of squares (SSLF) 
and can be decomposed (see Kutner, 2005, pages 124-125) into

$$
n^{-1} \operatorname{SSLF}(g)=\text { Error SS - Pure Error SS. }
$$

Note that (41) also takes into account the variance of the $n_{i}, i=1, \ldots, r$ observations corresponding to the same design point $x_{i}, i=1, \ldots, r$ around the regression estimate. This is often called "pure error". So we must subtract the pure error effect from (41) as follows:

$$
n^{-1} \operatorname{RSS}_{\text {distinct }}(g)=n^{-1} \sum_{i=1}^{r} \sum_{j=1}^{n_{i}}\left(Y_{i j}-g\left(x_{i}\right)\right)^{2}-n^{-1} \sum_{i=1}^{r} \sum_{j=1}^{n_{i}}\left(Y_{i j}-\bar{Y}_{i}\right)^{2},
$$

where $\bar{Y}_{i}=n_{i}^{-1} \sum_{j=1}^{n_{i}} Y_{i j}$ and

$$
\begin{aligned}
\sum_{i=1}^{r} \sum_{j=1}^{n_{i}}\left(Y_{i j}-g\left(x_{i}\right)\right)^{2} & =\sum_{i=1}^{r} \sum_{j=1}^{n_{i}}\left(Y_{i j}-\bar{Y}_{i}+\bar{Y}_{i}-g\left(x_{i}\right)\right)^{2} \\
& =\sum_{i=1}^{r} \sum_{j=1}^{n_{i}}\left(Y_{i j}-\bar{Y}_{i}\right)^{2}+\sum_{i=1}^{r} n_{i}\left(\bar{Y}_{i}-g\left(x_{i}\right)\right)^{2} \\
& +2 \sum_{i=1}^{r} \sum_{j=1}^{n_{i}}\left(Y_{i j}-\bar{Y}_{i}\right)\left(\bar{Y}_{i}-g\left(x_{i}\right)\right) .
\end{aligned}
$$

The cross-product term is equal to zero. Indeed,

$$
\begin{aligned}
\sum_{i=1}^{r} \sum_{j=1}^{n_{i}}\left(Y_{i j}-\bar{Y}_{i}\right)\left(\bar{Y}_{i}-g\left(x_{i}\right)\right)= & \sum_{i=1}^{r} \sum_{j=1}^{n_{i}}\left(Y_{i j} \bar{Y}_{i}-Y_{i j} g\left(x_{i}\right)-\bar{Y}_{i}^{2}+\bar{Y}_{i} g\left(x_{i}\right)\right) \\
= & \sum_{i=1}^{r} \bar{Y}_{i} \sum_{j=1}^{n_{i}} Y_{i j}-\sum_{i=1}^{r} g\left(x_{i}\right) \sum_{j=1}^{n_{i}} Y_{i j} \\
& -\sum_{i=1}^{r} n_{i} \bar{Y}_{i}^{2}+\sum_{i=1}^{r} n_{i} \bar{Y}_{i} g\left(x_{i}\right) .
\end{aligned}
$$


Using the fact that $\sum_{i=1}^{r} Y_{i j}=n_{i} \bar{Y}_{i}$,

$$
\begin{aligned}
& \sum_{i=1}^{r} \bar{Y}_{i} \sum_{j=1}^{n_{i}} Y_{i j}-\sum_{i=1}^{r} g\left(x_{i}\right) \sum_{j=1}^{n_{i}} Y_{i j}-\sum_{i=1}^{r} n_{i} \bar{Y}_{i}^{2}+\sum_{i=1}^{r} n_{i} \bar{Y}_{i} g\left(x_{i}\right) \\
& =\sum_{i=1}^{r} n_{i} \bar{Y}_{i}^{2}-\sum_{i=1}^{r} n_{i} g\left(x_{i}\right) \bar{Y}_{i}-\sum_{i=1}^{r} n_{i} \bar{Y}_{i}^{2}+\sum_{i=1}^{r} n_{i} g\left(x_{i}\right) \bar{Y}_{i} \\
& =0 .
\end{aligned}
$$

Therefore,

$$
\sum_{i=1}^{r} \sum_{j=1}^{n_{i}}\left(Y_{i j}-g\left(x_{i}\right)\right)^{2}=\sum_{i=1}^{r} \sum_{j=1}^{n_{i}}\left(Y_{i j}-\bar{Y}_{i}\right)^{2}+\sum_{i=1}^{r} n_{i}\left(\bar{Y}_{i}-g\left(x_{i}\right)\right)^{2} .
$$

And hence,

$$
n^{-1} R S S_{d i s t i n c t}(h)=n^{-1} \sum_{i=1}^{r} n_{i}\left(\bar{Y}_{i}-g\left(x_{i}\right)\right)^{2}
$$

Thus, we arrive at the following generalization of criterion (34) for non-distinct design points in model (35):

$$
\tilde{I}_{m}(g)=n^{-1} \sum_{i=1}^{r} n_{i}\left(\bar{Y}_{i}-g\left(x_{i}\right)\right)^{2}+\lambda \int_{0}^{1} g^{(m)}(x)^{2} d x
$$

Note that the second term is independent of the design points, and hence is not affected by the change.

Now, we will use Theorem 4.4 to find the estimator that minimizes $\tilde{I}_{m}(g)$. For this, consider the more general criterion that includes (43) as a special case:

$$
I_{\omega}(g)=n^{-1} \sum_{i=1}^{n} w_{i}\left(Y_{i}-g\left(x_{i}\right)\right)^{2}+\lambda \int_{0}^{1} g^{(m)}(x)^{2} d x
$$

where $\lambda$ is a positive parameter and $w_{1}, \ldots, w_{n}$ are positive constants (weights). Set- 
ting $w_{i}=n_{i}, i=1, \ldots, r$ and $w_{i}=0, i=r+1, \ldots, n$ gives (43). Criterion (44) can also be used to deal with heteroskedastic data, that is, data from model (35) where $\varepsilon_{i}$ are independent $N\left(0, \sigma_{i}^{2}\right)$ normal random errors with possibly different variances. To work with this model, one can use (44) with $w_{i}=\sigma_{i}^{-2}$. Note that Lemma 4.1 can also be applied to the minimization of (44), since this is still equivalent to choosing $g$ such that $g\left(x_{i}\right)=a_{i}$ and minimizing $J_{m}(g)=\int_{0}^{1}\left(g^{(m)}(x)\right)^{2} d x$ over all $f \in W_{2}^{m}[0,1]$. Therefore, by Lemma 4.1 , the unique minimizer of (44) is a natural spline $s=\sum_{j=1}^{n} b_{j} t_{j}$. The coefficients are obtained by the following theorem.

Theorem 4.4 (Reinsch, 1967). Let $q_{1}, \ldots, q_{n}$ be a basis for the set of natural splines of degree $2 m-1$ with knots $x_{1}, \ldots, x_{n}$ and define the matrix $\boldsymbol{Q}=\left(q_{j}\left(x_{i}\right)\right)_{n \times n}$. Then, if $n \geq m$ the unique minimizer of (44) is

$$
s=\sum_{j=1}^{n} d_{\lambda j} q_{j},
$$

where $\boldsymbol{d}=\left(d_{\lambda 1}, \ldots, d_{\lambda n}\right)^{\top}$ is the unique solution of the equation system

$$
\left(\mathbf{Q}^{\top} \mathbf{W} \mathbf{Q}+n \lambda \Omega\right) \mathbf{d}=\mathbf{Q}^{\top} \mathbf{W} \mathbf{Y}
$$

with $\boldsymbol{W}=\operatorname{diag}\left(w_{1}, \ldots, w_{n}\right)$.

Proof (Exercise 5.9.3 in Eubank, 1999). The proof is completely analogous to that of Theorem 4.3. The first term in (44) is minimized by choosing $g$ such that $g\left(x_{i}\right)=Y_{i}$. Therefore, (44) must be minimized over all functions of the form $s=\sum_{j=1}^{n} d_{j} q_{j}$, and the problem is reduced to minimizing

$$
\begin{aligned}
S_{\mathbf{W}}(\mathbf{d}) & =\frac{1}{n}(\mathbf{Y}-\mathbf{Q d})^{\top} \mathbf{W}(\mathbf{Y}-\mathbf{Q d})+\lambda \mathbf{d}^{\top} \mathbf{\Omega} \mathbf{d} \\
& =\frac{1}{n} \mathbf{Y}^{\top} \mathbf{W} \mathbf{Y}-\frac{2}{n} \mathbf{d}^{\top} \mathbf{Q}^{\top} \mathbf{W} \mathbf{Y}+\frac{1}{n} \mathbf{d}^{\top} \mathbf{Q}^{\top} \mathbf{W} \mathbf{Q d}+\lambda \mathbf{d}^{\top} \mathbf{\Omega} \mathbf{d}
\end{aligned}
$$


Using (38) and (39), the derivative of $S_{\mathbf{W}}(\mathbf{d})$ with respect to $\mathbf{d}$ is equal to

$$
\frac{d S_{\mathbf{W}}(\mathbf{d})}{d \mathbf{d}}=-\frac{2}{n} \mathbf{Q}^{\top} \mathbf{W} \mathbf{Y}+\frac{2}{n} \mathbf{Q}^{\top} \mathbf{W Q d}+2 \lambda \Omega \mathbf{d}
$$

Setting

$$
\frac{d S_{\mathbf{W}}(\mathbf{d})}{d \mathbf{d}}=0
$$

leads to the equation

$$
\mathbf{Q}^{\top} \mathbf{W} \mathbf{Y}=\mathbf{Q}^{\top} \mathbf{W Q d}+n \lambda \boldsymbol{\Omega d},
$$

or equivalently,

$$
\left(\mathbf{Q}^{\top} \mathbf{W Q}+n \lambda \Omega\right) \mathbf{d}=\mathbf{Q}^{\top} \mathbf{W} \mathbf{Y}
$$

Using the same reasoning as in the proof of Theorem 4.3, we may conclude that $\left(\mathbf{Q}^{\top} \mathbf{W Q}+n \lambda \Omega\right)$ is positive definite. Therefore, (45) has a unique solution. Theorem 4.4 is proved.

Note: When proving Theorem 4.4 we found a misprint in Theorem 5.3 of Eubank (1999), where the matrix $\mathbf{W}$ on the right hand side of equation (45) is omitted.

The estimator obtained in Theorem 4.4 generalizes the estimator obtained in Theorem 4.3. Recall that Theorem 4.3 has been used to fit a smoothing spline to a set of observations from model (35) with distinct design points. We shall now use Theorem 4.4 to obtain the spline smoothing estimator for model (40) with non-distinct design points.

Theorem 4.5. Let $q_{1}, \ldots, q_{m}$ be a basis for the set of natural splines of degree $2 m-1$ with distinct knots $x_{1}, \ldots, x_{m}$ and define $\boldsymbol{Q}=\left(q_{j}\left(x_{i}\right)\right)_{n \times n}$. Assume that 
the observations are $Y_{i j}=f\left(x_{i}\right)+\varepsilon_{i j}, i=1, \ldots, m, j=1, \ldots, n_{i}$. Then (44) is minimized when $g$ is a natural spline

$$
s=\sum_{i=1}^{m} d_{\lambda i} q_{i}
$$

where $\boldsymbol{d}=\left(d_{\lambda 1}, \ldots, d_{\lambda m}\right)^{\top}$ is the unique solution of the equation system

$$
\left(\mathbf{Q}^{\top} \mathbf{W} \mathbf{Q}+n \lambda \Omega\right) \mathbf{d}=\mathbf{Q}^{\top} \mathbf{W} \overline{\boldsymbol{Y}}
$$

with $\boldsymbol{W}=\operatorname{diag}\left(w_{1}, \ldots, w_{m}\right)$ and $\overline{\boldsymbol{Y}}=\left(\bar{Y}_{1}, \ldots, \bar{Y}_{m}\right)^{\top}$.

Let us return to the example of the liver function test as a predictor of survival times. In Figure 13, we fit a weighted smoothing spline estimator to the averaged responses for every level $X_{i}$ (with weights $w_{i}=n_{i}, i=1, \ldots, m$ ) using bandwidths obtained by the methods of cross-validation and generalized cross-validation discussed in Chapter 5. The resulting estimator suggests, as expected, that there is a positive relationship between the predictor and the response. In particular, we find that for low values of the liver function test score, the survival time increases with the test score at an increasing rate. Moreover, for high values of the liver function test score, the survival time increases with the test score at a decreasing rate. The residual plot and the QQ-plot are shown in Figures 14 and 15. From these we can conclude that, at least approximately, model (40) is satisfied, and the use of the weighted spline smoother is justified. 


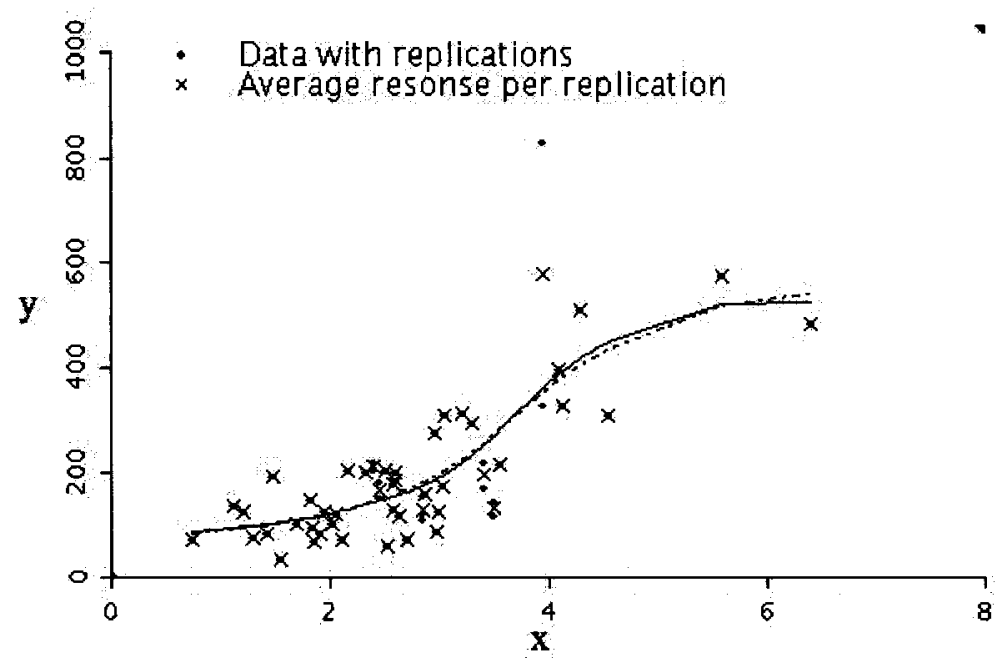

Figure 13: Liver function test scores $\left(X_{i}\right)$ versus survival times $\left(Y_{i}\right)$ of liver patients with weighted smoothing spline estimators (based on the averaged responses for every $X_{i}$ with weights $w_{i}=n_{i}$, $i=1, \ldots, m$ ). The smoothing parameters were chosen by the methods of cross-validation (estimator as solid line, $\lambda_{C V}=0.0019944384$ ) and generalized cross-validation (estimator as dotted line, $\lambda_{C V}=$ 0.003827022).

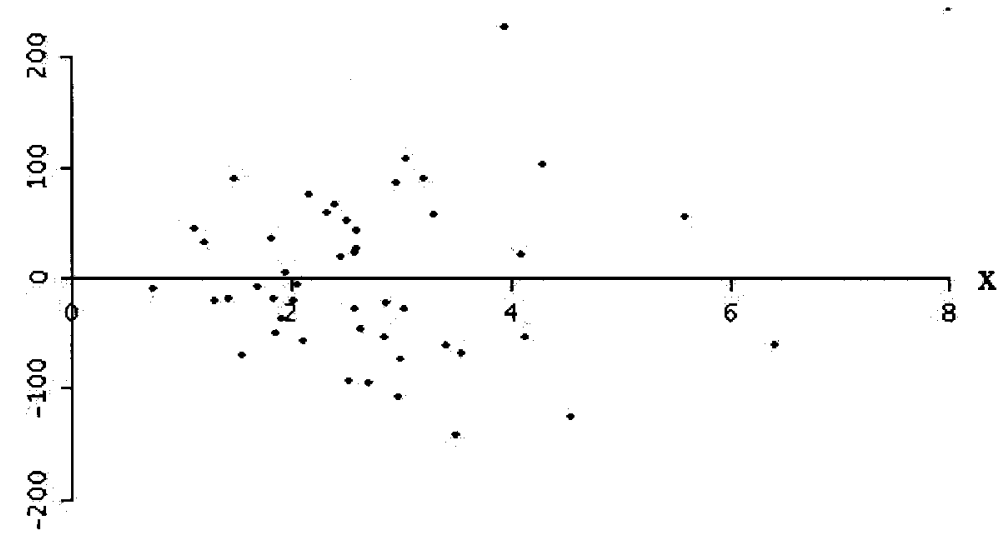

Figure 14: Residual plot for weighted smoothing spline estimator (based on the averaged responses for every $X_{i}$ with weights $w_{i}=n_{i}, i=1, \ldots, m$ ) with smoothing parameter $\lambda_{C V}=0.0019944384$. 


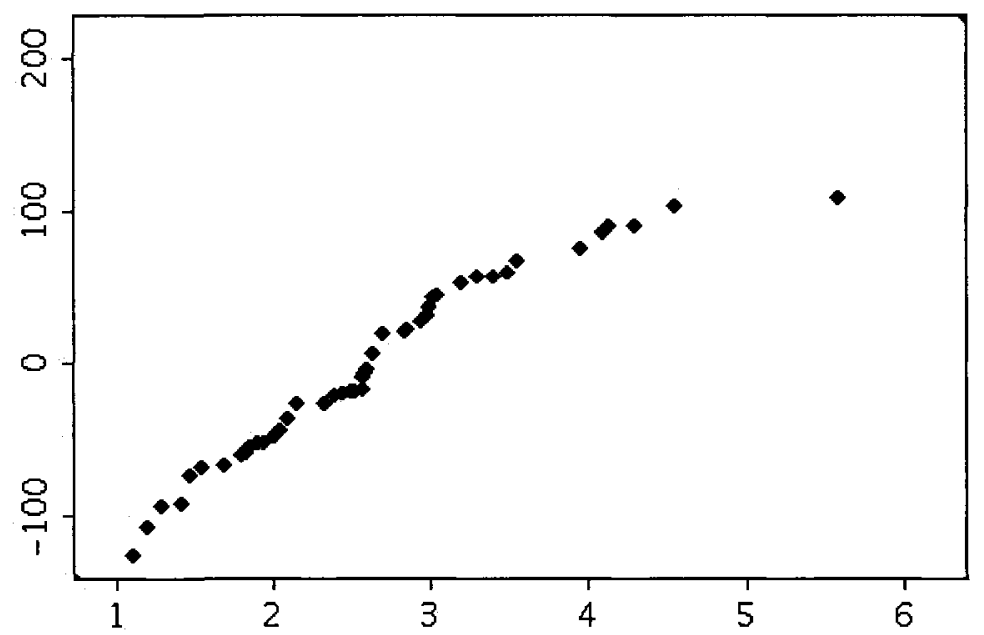

Figure 15: QQ-plot for weighted smoothing spline estimator (based on the averaged responses for every $X_{i}$ with weights $w_{i}=n_{i}, i=1, \ldots, m$ ) with smoothing parameter $\lambda_{C V}=0.0019944384$

Once again, consider model (35). Let us show that the vector of the spline smoothing estimator at the design points, $\mathbf{f}_{n}=\left(f_{n}\left(x_{1}\right), \ldots, f_{n}\left(x_{n}\right)\right)^{\top}$, can be written in the form

$$
\mathbf{f}_{n}=\mathbf{S}_{n} \mathbf{Y}
$$

where $\mathbf{S}_{n}$ is a symmetric, positive semi-definite $n \times n$ matrix. By Theorem 4.4 , the spline estimator has the form $f_{n}=\sum_{j=1}^{n} d_{\lambda j} q_{j}$, where $\mathbf{d}_{\lambda}=\left(d_{\lambda \mathbf{l}}, \ldots, d_{\lambda n}\right)^{\top}$ is the unique solution of the equation system

$$
\left(\mathbf{Q}^{\top} \mathbf{W Q}+n \lambda \Omega\right) \mathbf{d}=\mathbf{Q}^{\top} \mathbf{W} \mathbf{Y}
$$


Since $\left(\mathbf{Q}^{\top} \mathbf{W} \mathbf{Q}+n \lambda \boldsymbol{\Omega}\right)$ is nonsingular, we obtain

$$
\begin{gathered}
\mathbf{d}=\left(\mathbf{Q}^{\top} \mathbf{W} \mathbf{Q}+n \lambda \boldsymbol{\Omega}\right)^{-1} \mathbf{Q}^{\top} \mathbf{W} \mathbf{Y}, \\
\text { or equivalently } \\
\mathbf{Q} \mathbf{d}=\mathbf{Q}\left(\mathbf{Q}^{\top} \mathbf{W} \mathbf{Q}+n \lambda \Omega\right)^{-1} \mathbf{Q}^{\top} \mathbf{W} \mathbf{Y}
\end{gathered}
$$

Using the fact that $\mathbf{f}_{n}=\mathbf{Q d}$, we can write

$$
\mathbf{f}_{n}=\mathbf{S}_{n} \mathbf{Y}
$$

with symmetric and positive definite matrix $\mathbf{S}_{n}=\mathbf{Q}\left(\mathbf{Q}^{\top} \mathbf{W} \mathbf{Q}+n \lambda \Omega\right)^{-1} \mathbf{Q}^{\top} \mathbf{W}$.

In our previous discussion, the values of $m$ (the order of the Sobolev space $\left.W_{2}^{m}[0,1]\right)$ and $\lambda$ (the Lagrange multiplier) were assumed known. In practice, however, $\lambda$ and $m$ are rarely known and must be estimated form the data. It is possible to use any of the methods discussed in Chapter 5 to estimate $m$ from the data. However, this process can be computationally demanding. In practice, a spline of degree $m=3$ (cubic spline) is used. Theoretical results discussed in the next section show that, although increasing $m$ improves the smoother's performance to some extent, its benefit over using a cubic spline is very small. The choice of the smoothing parameter $\lambda$ does not pose computational difficulties, and can be estimated by using the methods of cross-validation or generalized cross-validation as shown in Chapter 5 . 


\subsection{Relationship to Other Regression Estimators}

As we have seen, a spline smoothing estimator is the unique solution to the minimization problem

$$
I(g) \longrightarrow \min _{g \in W_{2}^{m}[0,1]}
$$

where $I(g)$ is given by (34) (or (44) in the case of replications). Recall that the orthogonal series estimator $\tilde{f}_{n}$ discussed in Chapter 3 is just the estimated partial sum of the Fourier series of regression function $f$, which has the property of best approximation. Thus, both estimators are obtained as solutions to certain optimization problems. Therefore, it is not surprising that splines can be represented as orthogonal series with an appropriate choice of natural spline basis functions (as shown in the work of Demmler and Reinsch, 1975). Moreover, from the work of Silverman (1984) we see that under certain conditions, a smoothing spline estimator can be approximated by a kernel estimator. Therefore, in some cases, the choice of the type of regression estimator (kernel estimator, orthogonal series estimator, or smoothing spline) is not as crucial as the choice of the smoothing parameter.

Demmler and Reinsch (1975) showed that the spline smoothing estimator that minimizes (34) can be written in the form of a Demmler-Reinsch Fourier series using a basis $\mathcal{Q}=\left\{q_{1}, \ldots, q_{n}\right\}$ for $N S^{2 m-1}\left(x_{0}, \ldots, x_{n+1}\right)$ that has the following properties. First, $q_{1}, \ldots, q_{m}$ must span the space of $m$ th order polynomials, each basis function $q_{i}$ must have at least $i-1$ sign changes on the interval $(0,1), i=1, \ldots, m$. Besides this, the matrix $\mathbf{Q}=\left(q_{j}\left(x_{i}\right)\right)_{n \times n}$ must have the orthogonality property $\mathbf{Q}^{\top} \mathbf{Q}=n \mathbf{I}=\mathbf{Q Q}^{\top}$. The Demmler-Reinsch basis functions appear to admit a simple closed form only in the special case of $m=1$ and a uniform design. Also, put $\boldsymbol{\Omega}=\operatorname{diag}\left(0, \ldots, 0, \gamma_{1}, \ldots, \gamma_{n-m}\right)$, where $0<\gamma_{1} \leq \ldots \leq \gamma_{n-m}$ are constants defined 
in Demmler and Reinsch (1975) (they are known as Demmler-Reinsch eigenvalues). Under these conditions, the $m$ th order spline regression estimator can be written in the Demmler-Reinsch representation as

$$
f_{n}=\sum_{j=1}^{m} d_{j} q_{j}+\sum_{j=m+1}^{n} \frac{d_{j}}{1+\lambda \gamma_{j-m}} q_{j}
$$

as shown in Demmler and Reinsch (1975). That is, a spline regression estimator with appropriate choice of basis functions can be written as a weighted orthogonal series estimator with the sample Demmler-Rinsch Fourier coefficients

$$
d_{j}=n^{-1} \sum_{i=1}^{n} Y_{i} q_{j}\left(x_{i}\right), \quad j=1, \ldots, n
$$

and weights $\left(1+\lambda \gamma_{j-m}\right)^{-1}, j=m+1, \ldots, n$, called damping factors. Recall that for smoothing splines, the factor $\lambda$ controls the emphasis that is put on smoothness versus fit at the design points. In the Demmler-Reinsch representation $\lambda$ retains its meaning. Indeed, using the results of Nussbaum (1985), Speckman (1985) and Golubev and Nussbaum (1990), as $n \rightarrow \infty, \gamma_{j}$ can be written as

$$
\gamma_{j}=C(j \pi)^{2 m-1}(1+o(1))
$$

where $C$ is a constant that depends only on $m$ and on the type of design. Therefore, as $\lambda \rightarrow \infty$, the regression estimator reduces to $f_{n}=\sum_{j=1}^{m} d_{j} q_{j}$, an $m$ th degree polynomial that gives the smoothest fit to the data, that is, one that minimizes $J_{m}\left(f_{n}\right)$. Moreover, when $\lambda=0$, the regression function reduces to $f_{n}=\sum_{j=1}^{n} d_{j} q_{j}$, which is an interpolating polynomial that provides the fit to the data with the most agreement at the design points.

Silverman (1984) showed that, under certain smoothness conditions, a smoothing 
spline estimator essentially takes the form of a kernel estimator with a specific kernel function, as $n \rightarrow \infty$. More precisely, assume that the estimated function $f$ belongs to $W_{2}^{2}[0,1]$. Then, it is well-know (see equation (2.2) of Wahba, 1975) that the smoothing spline estimator can be written in the form,

$$
f_{n}(x)=n^{-1} \sum_{i=1}^{n} W_{n}\left(x, x_{i}\right) Y_{i},
$$

where the weight function $W_{n}(x, y)$ depends on the design points $x_{1}, \ldots, x_{n}$ and on the smoothing parameter $\lambda$. Theorem A from Silverman (1984) shows that, in particular, in the case of a uniform design, assuming that the smoothing parameter $\lambda=\lambda_{n}$ satisfies $\lambda \rightarrow 0$ and $\lambda n^{1-\varepsilon} \rightarrow \infty$, for some $\varepsilon>0$ and as $n \rightarrow \infty$, the weight function $W_{n}\left(x, x_{i}\right), i=1, \ldots, n$ will satisfy for all $x$ for which $x_{i}+\left(n^{-1} \lambda\right)^{1 / 4} x$ lies in $[0,1]$

$$
\left(n^{-1} \lambda\right)^{1 / 4} W_{n}\left(x_{i}+\left(n^{-1} \lambda\right)^{1 / 4} x, x_{i}\right) \approx K_{s}(x)
$$

where $K_{s}$ is the so-called spline kernel given by

$$
K_{s}(u)=\frac{1}{2} \exp \left(-\frac{|u|}{\sqrt{2}}\right) \sin \left(\frac{|u|}{\sqrt{2}}+\frac{\pi}{4}\right)
$$

The function $K_{s}$ is shown in Figure 16. It is straightforward to show that $K_{s}$ satisfies the usual assumptions on kernel functions. Using the transformation $s=x_{i}+x h$ in (47) with $h=\left(n^{-1} \lambda\right)^{1 / 4}$, for sufficiently large $n$ and small $\lambda$,

$$
W_{n}\left(s, x_{i}\right) \approx \frac{1}{h} K_{s}\left(\frac{s-x_{i}}{h}\right)
$$

In summary, Theorem A from Silverman (1984) implies that inside the interval $[0,1]$, spline weights are asymptotically equivalent to kernel weights. Figure 17 shows the 


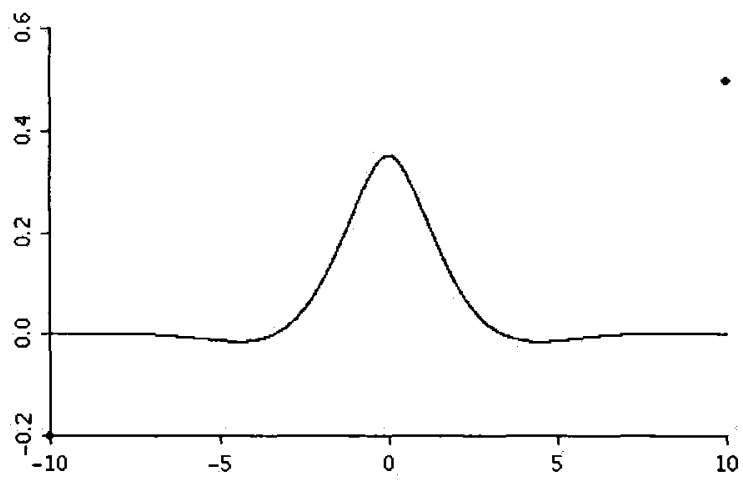

Figure 16: Spline kernel function $K_{s}(u)=\frac{1}{2} \exp \left(-\frac{|u|}{\sqrt{2}}\right) \sin \left(\frac{|u|}{\sqrt{2}}+\frac{\pi}{4}\right)$.

spline smoother and the kernel regression estimator with kernel $K_{s}$ and bandwidth $\left(n^{-1} \lambda\right)^{1 / 4}$ on the same plot. As predicted by Silverman, the two estimators nearly coincide inside the interval. The smoothing spline estimator exhibits better behaviour near the boundary than the kernel estimator.

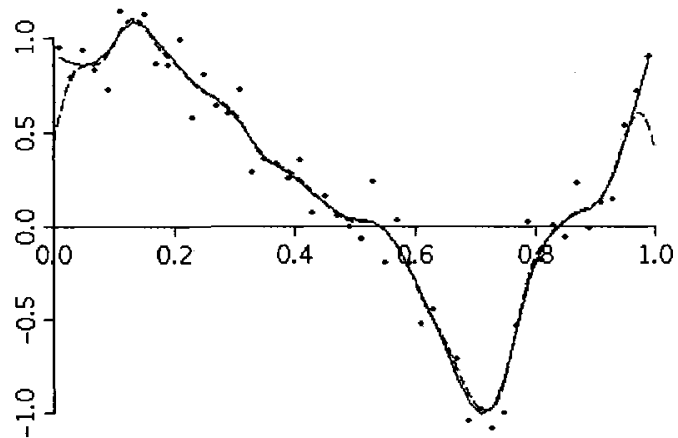

(a) $\lambda_{C V}=1.576048 \times 10^{-5}$

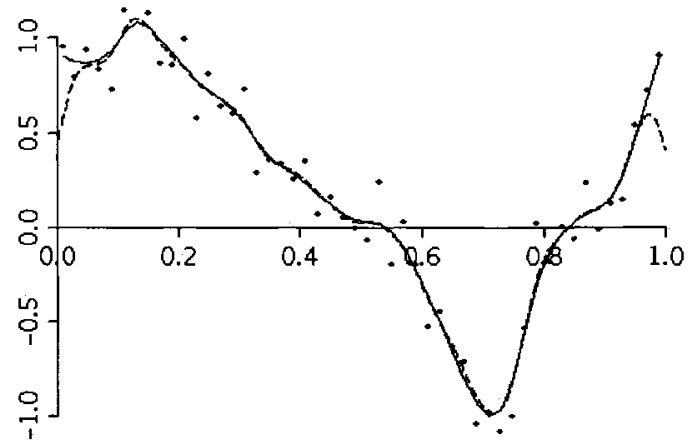

(b) $\lambda_{G C V}=1.757194 \times 10^{-5}$

Figure 17: A cubic smoothing spline (solid line) fit to $n=50$ data points using model $Y_{i}=$ $f\left(x_{i}\right)+\varepsilon_{i}$, where $\left\{x_{i}\right\}_{i=1}^{50}$ is a uniform design. The regression function is $f(x)=\cos ^{3}(2 \pi x)$ and the error terms $\varepsilon_{1}, \ldots, \varepsilon_{n}$ are randomly generated from an $N\left(0,(0.15)^{2}\right)$ normal distribution. The spline smoother was produced by the function smooth.spline() in S-Plus with smoothing parameter $\lambda$ chosen by the methods of cross-validation and generalized cross-validation, respectively. An equivalent spline kernel fit to the data (dotted line) uses a kernel regression estimator with the spine kernel $K_{s}$ and a bandwidth of $\left(n^{-1} \lambda\right)^{1 / 4}$, where $\lambda=\lambda_{C V}$ and $\lambda=\lambda_{G C V}$. 


\subsection{Asymptotic Performance}

Cox (1983) showed that when the true regression function is an element of $W_{2}^{m}[0,1]$, it is possible for a spline smoothing estimator obtained as a solution to the minimization problem

$$
n^{-1} \sum_{i=1}^{n}\left(Y_{i}-g\left(x_{i}\right)\right)^{2}+\lambda \int_{0}^{1} g^{(m)}(x)^{2} d x \rightarrow \min _{g \in W_{2}^{m}[0,1]}
$$

to attain the optimal rate of convergence $r_{n}=n^{-m /(2 m+1)}$ that was derived by Speckman (1985). That is, for $f \in W_{2}^{m}[0,1], m \geq 2$ and small $\lambda$

$$
L\left(f_{n}, f\right)=n^{-1} \sum_{k=1}^{n}\left(f\left(x_{k}\right)-f_{n}\left(x_{k}\right)\right)^{2}=O_{P}(\lambda)+O_{P}\left(n^{-1} \lambda^{-1 / 2 m}\right)
$$

In other words, the loss function decays to zero at the rate $O_{P}(\lambda)+O_{P}\left(n^{-1} \lambda^{-1 / 2 m}\right)$. Therefore, with the smoothing parameter chosen to be $\lambda=n^{-2 m /(2 m+1)}$, the loss (48) becomes

$$
L\left(f_{n}, f\right)=O_{P}\left(n^{-2 m /(2 m+1)}\right)
$$

which implies that

$$
R\left(f_{n}, f\right)=\mathbf{E} L\left(f_{n}, f\right)=O\left(n^{-2 m /(2 m+1)}\right)
$$

That is, the smoothing spline estimator attains the optimal rate of convergence.

However, a problem arises when we try to obtain an optimal smoothing spline estimator by setting the bandwidth to $\lambda_{n}=n^{-2 m /(2 m+1)}$, because $m$ may be unknown. Instead of having to select a value for $m$, cubic splines (degree $m=3$ ) are often used for spline smoothing, as they are known to perform well under many designs. 
Carter, Eagleson and Silverman (1992) investigated how the cubic spline would perform with respect to the optimal estimator of Speckman (1985) in terms of the rate of convergence. They found that, under certain conditions, the rate of convergence for the cubic smoothing spline is only $9 \%$ less efficient than that of Speckman's optimal estimator.

Another argument in favour of using cubic splines is provided by Theorem 5.25 of Cho (2007). This theorem deals with the problem of estimating an unknown regression function $f$ that belongs to the class $\mathcal{F}_{\gamma}(M)$ of analytic functions that are bounded in absolute value by a constant $M>0$ on a symmetric strip of size $2 \gamma$ around the real axis. For estimating $f \in \mathcal{F}_{\gamma}(M)$, Cho suggested using so-called C-spline estimators. These are kernel regression estimators with the kernel given by a fundamental cardinal spline of degree $m$ (order $m+1$ ). C-spline estimators are shown to be asymptotically optimal in the minimax sense if the degree $m$ satisfies $m \geq$ $\max \left(\pi \gamma s_{n}, 3\right)$, where $\gamma$ is the parameter involved in the definition of the functional class $\mathcal{F}_{\gamma}(M)$ and $s_{n}=(2 \pi \gamma)^{-1} \log \log n+O(1), n \rightarrow \infty$, is the asymptotically optimal bandwidth. Even for large sample sizes, $\max \left(\pi \gamma s_{n}, 3\right)=3$, which leads to a cubic smoothing spline.

The problem of choosing the smoothing parameter $\lambda$ adaptively, that is, by relying on the data only, will be discussed in Chapter 5 . 


\section{Choice of Smoothing Parameter}

As before, consider the fixed design model (1)

$$
Y_{i}=f\left(x_{i}\right)+\varepsilon_{i}, i=1, \ldots, n,
$$

where $f$ is an unknown regression function, $x_{i}$ are fixed design points chosen by the experimenter, and $\varepsilon_{i}$ are independent $N\left(0, \sigma^{2}\right)$ random variables. The quality of any of the nonparametric regression estimators of $f$ discussed in the thesis strongly depends on the choice of smoothing parameter, such as the bandwidth, $h$, in the case of kernel type estimators. Recall that the accuracy of estimation is often measured by the risk

$$
R\left(f_{n}, f\right)=n^{-1} \sum_{i=1}^{n} \mathbf{E}\left(f\left(x_{i}\right)-f_{n}\left(x_{i}\right)\right)^{2},
$$

where an estimator $f_{n}$ of $f$ depends on the smoothing parameter, which we will call $h$. This chapter addresses the important problem of choosing the parameter $h$. Therefore, from now on we will denote the risk by $R(h)$ rather than $R\left(f_{n}, f\right)$.

Ideally, given a regression estimator $f_{n}$, we would like to choose a smoothing parameter such that the resulting regression estimate is as close as possible to the true regression function $f$. Since we do not know the true regression function, our goal is to estimate the bandwidth $h_{\text {opt }}$ that minimizes the risk

$$
R(h)=n^{-1} \sum_{i=1}^{n} \mathbf{E}\left(f\left(x_{i}\right)-f_{n}\left(x_{i}\right)\right)^{2},
$$

or the related prediction risk criterion

$$
P(h)=n^{-1} \sum \mathbf{E}\left(Y_{i}^{*}-f_{n}\left(x_{i}\right)\right)^{2},
$$


where $Y_{i}^{*}$ is a future observation from the regression model $Y_{i}^{*}=f_{n}\left(x_{i}\right)+\varepsilon_{i}^{*}$, with $\varepsilon_{i}^{*}$ independent of each other and independent of the normal $N\left(0, \sigma^{2}\right)$ random errors $\varepsilon_{i}$. It will be shown that merely choosing the bandwidth that minimizes the unbiased prediction risk criterion requires the knowledge of the error term variance $\sigma^{2}$. As this is not usually available in practice, we must base our choice of $h$ on the sample only, that is, by using an adaptive method. Two such methods will be discussed, namely, the method of cross-validation (CV) and the method of generalized cross-validation (GCV). The method of cross-validation involves calculating an estimate of the prediction risk that is nearly unbiased in the first place and so does not require a bias correction term that depends on $\sigma^{2}$. The method of generalized cross-validation aims to reduce the bias for estimating the prediction risk by a correction factor and is easier to compute. It will be shown that CV and GCV are often asymptotically equivalent. Moreover, it will be shown that, for large $n$, the bandwidth minimizing either $C V(h)$, or $G C V(h)$, or the unbiased risk criterion will tend to the optimal bandwidth at the rate of $n^{-1 / 5}$.

It will now be convenient to consider model (1) in matrix notation. Recall that

$$
\begin{gathered}
\mathbf{Y}=\left(Y_{1}, \ldots, Y_{n}\right)^{\top}, \\
\mathbf{f}=\left(f\left(x_{1}\right), \ldots, f\left(x_{n}\right)\right)^{\top} \\
\boldsymbol{\varepsilon}=\left(\varepsilon_{1}, \ldots, \varepsilon_{n}\right)^{\top} .
\end{gathered}
$$

Then model (1) takes the form

$$
\mathbf{Y}=\mathbf{f}+\varepsilon
$$


We are interested in estimating the true regression function $f(x)$ by an estimator $f_{n}(x)=f_{n}(\mathbf{Y}, x, h)$ from the class of all linear estimators. The most popular regression estimators, such as kernel estimators, projection estimators and smoothing splines, belong to this class. Recall that a linear estimator of the regression function at point $x$ has the form

$$
f_{n}(x)=\sum_{i=1}^{n} k_{n}\left(x, x_{i} ; h\right) Y_{i}
$$

where $k_{n}\left(x, x_{i} ; h\right)$ is a weight function and $h=h_{n}$ is a smoothing parameter that depends on $n$. The estimator $\mathbf{f}_{n}=\left(f_{n}\left(x_{1}\right), \ldots, f_{n}\left(x_{n}\right)\right)^{\top}$ of $\mathbf{f}=\left(f\left(x_{1}\right), \ldots, f\left(x_{n}\right)\right)^{\top}$ can be written in the form

$$
\mathbf{f}_{n}=\mathbf{S}_{n} \mathbf{Y}
$$

where

$$
\mathbf{S}_{n}=\left(\mathbf{S}_{i j}\right)_{i, j=1}^{n}, \quad \mathbf{S}_{i j}=\mathbf{S}_{i j}^{(n)}=k_{n}\left(x_{i}, x_{j} ; h\right)
$$

In order to guarantee convergence of the estimator to the regression function in mean square error, certain assumptions on the smoothing parameter are needed. For example, for kernel type estimators we have assumed that the bandwidth $h$ satisfies, as $n \rightarrow \infty$,

$$
h \rightarrow 0, \quad n h \rightarrow \infty
$$

Similarly, in the case of smoothing splines, we have assumed that the smoothing parameter $\lambda$, that balances smoothness versus fit, satisfies, as $n \rightarrow \infty$,

$$
\lambda \rightarrow 0, \quad n \lambda \rightarrow \infty
$$

Eubank (1999) shows that in some cases, the weaker assumption $n^{1-\delta} \lambda \rightarrow \infty$ for 
some $\delta \in(0,1)$ is enough to guarantee convergence of the estimator to the regression function in mean square error. In the case of projection estimators, we assume that the parameter $N$ that defines the partial sum of the Fourier series used for estimating $f(x)$ satisfies, as $n \rightarrow \infty$,

$$
N \rightarrow \infty, \quad N n^{-1} \rightarrow 0
$$

In some interesting cases, the parameters $h$ and $N$, that determine the optimal kernel estimator and the optimal projection estimator respectively, are approximately inversely proportional to each other.

Typically, optimal bandwidths that arise in problems of estimating regression functions of finite smoothness are of order $n^{-\delta}$ for some $\delta \in(0,1)$ that depends on the smoothness conditions on $f$. Therefore, when dealing with kernel type estimators, following Hall, Härdle, and Marron (1988), we define the set of possible bandwidths to be

$$
H_{n}=\left\{h=O\left(n^{-1+\delta}\right), \quad \text { for some } \quad \delta \in(0,1)\right\} .
$$

Clearly, any bandwidth $h$ that belongs to $H_{n}$ satisfies (50).

The goal of the statistician is to select from the parameter space $H_{n}$ a "good" smoothing parameter $h$, that is, one that leads to low values of the risk $R(h)$ and of the prediction risk $P(h)$, where

$$
R(h)=n^{-1} \sum_{i=1}^{n} \mathbf{E}\left(f\left(x_{i}\right)-f_{n}\left(x_{i}\right)\right)^{2}
$$

and

$$
P(h)=n^{-1} \sum \mathbf{E}\left(Y_{i}^{*}-f_{n}\left(x_{i}\right)\right)^{2} .
$$

Define the optimal bandwidth $h_{\text {opt }}$ as a bandwidth that minimizes a given performance criterion. Ideally, we would like to obtain an estimate $\hat{h}_{\text {opt }}$ that converges as fast as 
possible to the optimal bandwidth.

Calculation of performance criterion (51) requires the knowledge of the regression function, which is not known in practice. Therefore, we must select a performance criterion that has an intuitively plausible and easily computable estimator. For this reason, the prediction risk

$$
P(h)=n^{-1} \sum \mathbf{E}\left(Y_{i}^{*}-f_{n}\left(x_{i}\right)\right)^{2}
$$

is often used as a performance measure. The average regression sum of squares has a similar form to the prediction risk, but is based on $n$ past, instead of future, observations. For this reason the prediction risk can be estimated by the average regression sum of squares given by

$$
n^{-1} R S S(h)=n^{-1} \sum_{i=1}^{n}\left(Y_{i}-f_{n}\left(x_{i}\right)\right)^{2}
$$

Note that $R S S(h)$ can be represented as a quadratic form in $\mathbf{Y}$ :

$$
\begin{aligned}
R S S(h) & =\left(\mathbf{Y}-\mathbf{f}_{n}\right)^{\top}\left(\mathbf{Y}-\mathbf{f}_{n}\right)=\left(\mathbf{Y}-\mathbf{S}_{n} \mathbf{Y}\right)^{\top}\left(\mathbf{Y}-\mathbf{S}_{n} \mathbf{Y}\right) \\
& =\mathbf{Y}^{\top}\left(\mathbf{I}-\mathbf{S}_{n}\right)^{\top}\left(\mathbf{I}-\mathbf{S}_{n}\right) \mathbf{Y}=\mathbf{Y}^{\top}\left(\mathbf{I}-\mathbf{S}_{n}\right)^{2} \mathbf{Y}
\end{aligned}
$$

where $\left(\mathbf{I}-\mathbf{S}_{n}\right)^{2}$ is a symmetric $n \times n$ matrix. Then, using (2), (49), and recalling 
that $\mathbf{E} \varepsilon=\mathbf{0}$, the expected value of this statistic is

$$
\begin{gathered}
\mathbf{E}\left(n^{-1} R S S(h)\right) \\
=n^{-1} \mathbf{E}\left((\mathbf{f}+\boldsymbol{\varepsilon})^{\top}\left(\mathbf{I}-\mathbf{S}_{n}\right)^{2}(\mathbf{f}+\boldsymbol{\varepsilon})\right) \\
=n^{-1}\left[\mathbf{E}\left(\mathbf{f}^{\top}\left(\mathbf{I}-\mathbf{S}_{n}\right)^{2} \mathbf{f}\right)+\mathbf{E}\left(\mathbf{f}^{\top}\left(\mathbf{I}-\mathbf{S}_{n}\right)^{2} \boldsymbol{\varepsilon}\right)+\mathbf{E}\left(\boldsymbol{\varepsilon}^{\top}\left(\mathbf{I}-\mathbf{S}_{n}\right)^{2} \mathbf{f}\right)+\mathbf{E}\left(\boldsymbol{\varepsilon}^{\top}\left(\mathbf{I}-\mathbf{S}_{n}\right)^{2} \varepsilon\right)\right] \\
=n^{-1}\left[\mathbf{f}^{\top}\left(\mathbf{I}-\mathbf{S}_{n}\right)^{2} \mathbf{f}+\mathbf{f}^{\top}\left(\mathbf{I}-\mathbf{S}_{n}\right)^{2} \mathbf{E}(\boldsymbol{\varepsilon})+\mathbf{E}\left(\boldsymbol{\varepsilon}^{\top}\right)\left(\mathbf{I}-\mathbf{S}_{n}\right)^{2} \mathbf{f}+\mathbf{E}\left(\boldsymbol{\varepsilon}^{\top}\left(\mathbf{I}-\mathbf{S}_{n}\right)^{2} \boldsymbol{\varepsilon}\right)\right] \\
=n^{-1}\left[\mathbf{f}^{\top}\left(\mathbf{I}-\mathbf{S}_{n}\right)^{2} \mathbf{f}+\mathbf{E}\left(\varepsilon^{\top}\left(\mathbf{I}-\mathbf{S}_{n}\right)^{2} \varepsilon\right)\right] .
\end{gathered}
$$

Next, using $\mathbf{E} \boldsymbol{\varepsilon} \boldsymbol{\varepsilon}^{\top}=\sigma^{2} \mathbf{I}$ and applying the multivariate property B1 (see Appendix) to the last term, we get

$$
\begin{aligned}
\mathbf{E}\left(n^{-1} R S S(h)\right) & =n^{-1} \mathbf{f}^{\top}\left(\mathbf{I}-\mathbf{S}_{n}\right)^{2} \mathbf{f}+n^{-1} \operatorname{tr}\left[\sigma^{2} \mathbf{I}\left(\mathbf{I}-\mathbf{S}_{n}\right)^{2}\right] \\
& =n^{-1} \mathbf{f}^{\top}\left(\mathbf{I}-\mathbf{S}_{n}\right)^{2} \mathbf{f}+n^{-1} \sigma^{2} \operatorname{tr}\left[\left(\mathbf{I}-\mathbf{S}_{n}\right)^{2}\right] \\
& =n^{-1} \mathbf{f}^{\top}\left(\mathbf{I}-\mathbf{S}_{n}\right)^{2} \mathbf{f}+\sigma^{2}+n^{-1} \sigma^{2} \operatorname{tr}\left[\mathbf{S}_{n}^{2}\right]-2 n^{-1} \sigma^{2} \operatorname{tr}\left[\mathbf{S}_{n}\right]
\end{aligned}
$$

To establish the relationship between $\mathbf{E}\left(n^{-1} R S S(h)\right)$ and $P(h)$, recall from Chapter 1 that the prediction risk is related to the risk $R(h)$ through the relation

$$
P(h)=\sigma^{2}+R(h) .
$$


Therefore, an alternative representation of the prediction risk $P(h)$ is

$$
\begin{gathered}
P(h)=\sigma^{2}+n^{-1} \mathbf{E}\left[\sum_{i=1}^{n}\left(f\left(x_{i}\right)-f_{n}\left(x_{i}\right)\right)^{2}\right] \\
=\sigma^{2}+n^{-1} \mathbf{E}\left(\mathbf{f}-\mathbf{f}_{n}\right)^{\top}\left(\mathbf{f}-\mathbf{f}_{n}\right)=\sigma^{2}+n^{-1} \mathbf{E}\left(\mathbf{f}-\mathbf{S}_{n} \mathbf{Y}\right)^{\top}\left(\mathbf{f}-\mathbf{S}_{n} \mathbf{Y}\right) \\
=\sigma^{2}+n^{-1} \mathbf{E}\left[\left(\mathbf{f}-\mathbf{S}_{n}(\mathbf{f}+\boldsymbol{\varepsilon})\right)^{\top}\left(\mathbf{f}-\mathbf{S}_{n}(\mathbf{f}+\boldsymbol{\varepsilon})\right)\right] \\
=\sigma^{2}+n^{-1} \mathbf{E}\left(\mathbf{f}^{\top}\left(\mathbf{I}-\mathbf{S}_{n}\right)^{\top}\left(\mathbf{I}-\mathbf{S}_{n}\right) \mathbf{f}-\mathbf{f}^{\top}\left(\mathbf{I}-\mathbf{S}_{n}\right)^{\top} \mathbf{S}_{n} \boldsymbol{\varepsilon}-\boldsymbol{\varepsilon}^{\top} \mathbf{S}_{n}^{\top}\left(\mathbf{I}-\mathbf{S}_{n}\right) \mathbf{f}+\boldsymbol{\varepsilon}^{\top} \mathbf{S}_{n}^{\top} \mathbf{S}_{n} \varepsilon\right) \\
=\sigma^{2}+n^{-1} \mathbf{f}^{\top}\left(\mathbf{I}-\mathbf{S}_{n}\right)^{2} \mathbf{f}+n^{-1} \mathbf{E} \boldsymbol{\varepsilon}^{\top} \mathbf{S}_{n}^{2} \varepsilon .
\end{gathered}
$$

From this, using property B1 from the Appendix, we obtain

$$
\begin{aligned}
P(h) & =\sigma^{2}+n^{-1} \mathbf{f}^{\top}\left(\mathbf{I}-\mathbf{S}_{n}\right)^{2} \mathbf{f}+n^{-1} \operatorname{tr}\left[\mathbf{I} \sigma^{2} \mathbf{S}_{n}^{2}\right] \\
& =\sigma^{2}+n^{-1} \mathbf{f}^{\top}\left(\mathbf{I}-\mathbf{S}_{n}\right)^{2} \mathbf{f}+n^{-1} \sigma^{2} \operatorname{tr}\left[\mathbf{S}_{n}^{2}\right]
\end{aligned}
$$

Combining this identity with (53) shows that the estimator $n^{-1} R S S(h)$ is biased for $P(h)$ :

$$
\begin{aligned}
\mathbf{E}\left(n^{-1} R S S(h)\right) & =n^{-1} \mathbf{f}^{\top}\left(\mathbf{I}-\mathbf{S}_{n}\right)^{2} \mathbf{f}+\sigma^{2}+n^{-1} \sigma^{2} \operatorname{tr}\left[\mathbf{S}_{n}^{2}\right]-2 n^{-1} \sigma^{2} \operatorname{tr}\left[\mathbf{S}_{n}\right] \\
& =P(h)-2 n^{-1} \sigma^{2} \operatorname{tr}\left[\mathbf{S}_{n}\right] .
\end{aligned}
$$

For given $n$, the estimator $n^{-1} R S S(h)$ tends to underestimate the prediction risk by $2 n^{-1} \sigma^{2} \operatorname{tr}\left[\mathbf{S}_{n}\right]$, a term that depends on $h$ and will therefore lead to a biased estimator of the optimal bandwidth. Intuitively, this downward bias can be explained by the fact that for each $i, Y_{i}$ is used in the estimator of the regression function, $f_{n}\left(x_{i}\right)$, to which it is compared. That is, each $Y_{i}$ is used to predict itself, and hence the estimator's performance will tend to be superior to the performance measured by the prediction risk which is based on new observations of $Y$. 
When $\sigma^{2}$ is known, it is possible to correct for the bias of $n^{-1} R S S(h)$. That is, the optimal bandwidth can be estimated by the bandwidth that minimizes the following unbiased estimator of the prediction risk from among a set of bandwidths chosen by the experimenter:

$$
\hat{h}_{\text {opt }}=\underset{h}{\operatorname{argmin}} \hat{P}(h),
$$

where

$$
\hat{P}(h)=n^{-1} R S S(h)+2 n^{-1} \sigma^{2} \operatorname{tr}\left[\mathbf{S}_{n}\right]
$$

or equivalently, by (54), one that minimizes the unbiased estimator for the risk

$$
\hat{R}(h)=\hat{P}(h)-\sigma^{2} .
$$

The above are called unbiased prediction risk criterion and unbiased risk criterion respectively. In practice, $\sigma^{2}$ is not usually known, so estimators (56) and (57) cannot be used. In such cases, an appropriate method of choosing $h$ must somehow "adapt" to the unknown parameters in our model. Such methods are often called adaptive. We will consider two adaptive methods in detail: (i) leave-one-out crossvalidation (CV) and (ii) generalized cross-validation (GCV).

The method of leave-one-out cross-validation prescribes to calculate the value of $C V(h)$, a modified version of the average squared error (52), at a set of bandwidths chosen by the experimenter:

$$
\begin{aligned}
C V(h) & =n^{-1} \sum_{i=1}^{n}\left\{Y_{i}-f_{n,-i}\left(x_{i}\right)\right\}^{2} \\
& =n^{-1}\left(\mathbf{Y}-\mathbf{f}_{h,-i}\right)^{\top}\left(\mathbf{Y}-\mathbf{f}_{h,-i}\right)
\end{aligned}
$$


where

$$
f_{n,-i}(x)=\sum_{\substack{j=1 \\ j \neq i}}^{n} k_{n}\left(x, x_{j}, h\right) Y_{j},
$$

is the leave-one-out estimator of $f(x)$ and

$$
\mathbf{f}_{h,-i}=\left(f_{n,-i}\left(x_{1}\right), \ldots, f_{n,-i}\left(x_{n}\right)\right)^{\top}
$$

The quantity $C V(h)$ is a modification of $n^{-1} R S S$ obtained by removing the $i$ th observation from the estimator $f_{n}$. This ensures that $Y_{i}$ is not used to predict itself in order to partially remove bias. The bandwidth $\hat{h}_{C V}$ that minimizes the crossvalidation score (58) over $H_{n}$ is taken as an estimate of the optimal bandwidth:

$$
\hat{h}_{C V}=\underset{h \in H_{n}}{\operatorname{argmin}} C V(h)
$$

Another popular method for choosing a smoothing parameter is the generalized cross-validation method, or $G C V$, proposed by Craven and Wahba (1979). The generalized cross-validation score consists of the statistic $n^{-1} R S S(h)$ multiplied by a penalty factor that corrects the downward bias caused by the bias term in expansion (55). Assuming that $\operatorname{tr}\left[\mathbf{S}_{n}\right]<n$, the $G C V$ criterion is given by,

$$
G C V(h)=n^{-1} R S S(h)\left(n^{-1} \operatorname{tr}\left[\mathbf{I}-\mathbf{S}_{n}\right]\right)^{-2} .
$$

Let us compute the quantity $G C V(h)$ for some specific estimators. First, consider the kernel regression estimator

$$
f_{n}(x)=n^{-1} \sum_{i=1}^{n} K_{h}\left(x-x_{i}\right) Y_{i}
$$


where $K_{h}(x)=h^{-1} K\left(h^{-1} x\right)$ and $K(x)$ satisfies

$$
\begin{gathered}
K(x)=K(-x), \\
\max _{x \in \mathbb{R}} K(x)=K(0), \\
\int_{-\infty}^{\infty} K^{2}(x) d x<\infty .
\end{gathered}
$$

In this case, the elements of $\mathbf{S}_{n}=\left(\mathbf{S}_{i j}\right)_{i, j=1}^{n}$ can be written as follows

$$
\mathbf{S}_{i j}=k_{n}\left(x_{i}, x_{j} ; h\right)=n^{-1} K_{h}\left(x_{i}-x_{j}\right)
$$

In particular,

$$
k_{n}\left(x_{i}, x_{i} ; h\right)=(n h)^{-1} K(0), \quad i=1, \ldots, n
$$

and hence the diagonal elements of $\mathbf{S}_{n}$ are equal to

$$
\mathbf{S}_{i i}=(n h)^{-1} K_{n}(0), \quad i=1, \ldots, n
$$

Thus, for the kernel estimator $f_{n}$, the $G C V$ criterion is given by

$$
\begin{aligned}
G C V(h) & =n^{-1} R S S(h)\left(n^{-1} \operatorname{tr}\left[\mathbf{I}-\mathbf{S}_{n}\right]\right)^{-2} \\
& =n^{-1} R S S(h)\left(n^{-1} \sum_{i=1}^{n}\left(1-\mathbf{S}_{i i}\right)\right)^{-2} \\
& =n^{-1} R S S(h)\left(n^{-1} \sum_{i=1}^{n}\left(1-(n h)^{-1} K_{n}(0)\right)\right)^{-2} \\
& =n^{-1} R S S(h)\left(1-(n h)^{-1} K_{n}(0)\right)^{-2} .
\end{aligned}
$$

Next, consider the smoothing spline $f_{n}$ as an estimator of $f$. Recall that $f_{n}$ is the 
solution to the minimization problem

$$
I_{w}(g)=n^{-1} \sum_{i=1}^{n} w_{i}\left(Y_{i}-g\left(x_{i}\right)\right)^{2}+\lambda \int_{0}^{1}\left(g^{(m)}(x)\right)^{2} d x \rightarrow \min _{g \in W_{2}^{m}[0,1]}
$$

The quality of $f_{n}$ is largely controlled by the parameter $\lambda$ whose role is somewhat similar to the role of the bandwidth $h$ in kernel estimation. As shown in Chapter 4, the vector $\mathbf{f}_{n}$ of the smoothing spline estimator evaluated at the design points can be written in the form

$$
\mathbf{f}_{n}=\mathbf{S}_{n} \mathbf{Y}
$$

where the matrix $\mathbf{S}_{n}$ is given by

$$
\mathbf{S}_{n}=\mathbf{Q}\left(\mathbf{Q}^{\top} \mathbf{W} \mathbf{Q}+n \lambda \boldsymbol{\Omega}\right)^{-1} \mathbf{Q}^{\top} \mathbf{W}
$$

with $\mathbf{Q}=\left(q_{j}\left(x_{i}\right)\right)_{n \times n}$ being a matrix of basis functions for the natural spline space of degree $2 m-1, \boldsymbol{\Omega}=\left(\int_{0}^{1} q_{i}^{(m)}(x) q_{j}^{(m)}(x) d x\right)_{n \times n}$, and $\mathbf{W}=\operatorname{diag}\left\{w_{1}, \ldots, w_{n}\right\}$ being the vector of weights from (62). However, it is hard to write the functions $\mathbf{S}_{i j}=$ $k_{n}\left(x_{i}, x_{j} ; \lambda\right)$ explicitly. It turns out that their dependence of the parameter $\lambda$ and the design is very complicated. Despite this difficulty, there are computational methods of evaluating the spline smoother weights $\mathbf{S}_{i j}=k_{n}\left(x_{i}, x_{j}, \lambda\right)$ efficiently for a given data set (see, for example, Section XIV of De Boor, 1978). One may use such methods to calculate the $G C V$ criterion from

$$
G C V(\lambda)=n^{-1} R S S(\lambda)\left(n^{-1} \operatorname{tr}\left[\mathbf{I}-\mathbf{Q}\left(\mathbf{Q}^{\top} \mathbf{W} \mathbf{Q}+n \lambda \mathbf{\Omega}\right)^{-1} \mathbf{Q}^{\top} \mathbf{W}\right]\right)^{-2}
$$

Alternatively, one may approximate the smoothing spline weights $\mathbf{S}_{i j}=k_{n}\left(x_{i}, x_{j} ; \lambda\right)$ 
by the weights given by the equivalent spline kernel representation from Silverman (1984), as discussed in Chapter 4, and use them to calculate the $G C V$ score obtained for the kernel regression method. Finally, consider the projection estimator

$$
\tilde{f}_{n}(x)=\sum_{k=0}^{N-1} \tilde{c}_{k} \phi_{k}(x)
$$

where $\left\{\phi_{k}\right\}_{k=0}^{\infty}$ is a complete double-orthogonal system with respect to the design $\left\{x_{i}\right\}_{i=1}^{n}$ and $\tilde{c}_{k}$ is an unbiased estimator of the $k$ th Fourier coefficient $c_{k}, k=1, \ldots, N-$ 1. The derivation at the end of Chapter 3 shows that the elements of $\mathbf{S}_{n}=\left(\mathbf{S}_{i j}\right)_{i, j=1}^{n}$ are equal to

$$
\mathbf{S}_{i j}=n^{-1} \sum_{k=0}^{N-1} \phi_{k}\left(x_{i}\right) \phi_{k}\left(x_{j}\right) .
$$

Hence, for the projection estimator $\tilde{f}_{n}(x)$, the $G C V$ criterion takes the form

$$
\begin{aligned}
G C V(N) & =n^{-1} R S S(N)\left(n^{-1} \operatorname{tr}\left[\mathbf{I}-\mathbf{S}_{n}\right]\right)^{-2} \\
& =n^{-1} R S S(N)\left(n^{-1} \sum_{i=1}^{n}\left(1-\mathbf{S}_{i i}\right)\right)^{-2} \\
& =n^{-1} R S S(N)\left(n^{-1} \sum_{i=1}^{n}\left(1-n^{-1} \sum_{k=0}^{N-1} \phi_{k}^{2}\left(x_{i}\right)\right)\right)^{-2} \\
& =n^{-1} R S S(N)\left(1-n^{-1} \sum_{k=0}^{N-1} n^{-1} \sum_{i=1}^{n} \phi_{k}^{2}\left(x_{i}\right)\right)^{-2}
\end{aligned}
$$

where $n^{-1} \sum_{i=1}^{n} \phi_{k}^{2}\left(x_{i}\right)=1$ due to the double-orthonormality of the system $\left\{\phi_{k}\right\}_{k=0}^{\infty}$ with respect to the design points $\left\{x_{i}\right\}_{i=1}^{n}$, and so, 


$$
n^{-1} R S S(N)\left(1-n^{-1} \sum_{k=0}^{N-1} n^{-1} \sum_{i=1}^{n} \phi_{k}^{2}\left(x_{i}\right)\right)^{-2}=n^{-1} R S S(N)\left(1-\frac{N}{n}\right)^{-2}
$$

Therefore, for the projection estimator $\tilde{f}_{n}(x)$,

$$
G C V(N)=n^{-1} R S S(N)\left(1-\frac{N}{n}\right)^{-2}
$$

Since $n^{-1} N \rightarrow 0$, as $n \rightarrow \infty$, the correction factor $\left(1-n^{-1} N\right)^{-2}$ tends to 1 , as $n$ tends to infinity.

The following theorem of Craven and Wahba (1979) shows that the generalized cross-validation score $G C V(h)$ is nearly an unbiased estimator of the prediction risk $P(h)$

Theorem 5.1 (Craven and Wahba, 1979). Let $\tau_{j}(h)=n^{-1} \operatorname{tr}\left[\mathbf{S}_{n}^{j}\right], j=1,2$, and assume that $\tau_{\mathbf{1}}(h)<1$. Then,

$$
|\mathbf{E G C V}(h)-P(h)| / R(h) \leq g(h)
$$

where

$$
g(h)=\left[2 \tau_{1}(h)+\tau_{1}(h)^{2} / \tau_{2}(h)\right] /\left(1-\tau_{1}(h)\right)^{2} .
$$

Now, let us compare the performance of the bandwidth selectors $C V(h)$ and $G C V(h)$ for the estimators studied in this thesis. First, consider the kernel estimator

$$
f_{n}(x)=n^{-1} \sum_{i=1}^{n} K_{h}\left(x-x_{i}\right) Y_{i}=(n h)^{-1} \sum_{i=1}^{n} K\left(h^{-1}\left(x-x_{i}\right)\right) Y_{i}
$$

It is well-known (see, for example, Härdle, Hall, and Marron, 1988) that under certain 
smoothness assumptions on $f$ and $K$, the cross-validation and generalized crossvalidation scores, $C V(h)$ and $G C V(h)$, satisfy

$$
\frac{G C V(h)}{n^{-1} R S S(h)}=1+2 n^{-1} h^{-1} K(0)+O_{P}\left(n^{-2} h^{-2}\right)
$$

and

$$
\frac{C V(h)}{n^{-1} R S S(h)}=1+2 n^{-1} h^{-1} K(0)+O_{P}\left(n^{-2} h^{-2}\right),
$$

uniformly over $h \in H_{n}$. Inspection of the proofs of these two results shows that they continue to hold, with some constant $c$ in the place of $K(0)$, for the linear estimator

$$
f_{n}(x)=\sum_{i=1}^{n} k_{n}\left(x, x_{i} ; h\right) Y_{i}=\frac{1}{n} \sum_{i=1}^{n} K_{n}\left(x, x_{i} ; h\right) Y_{i}
$$

for which

$$
K_{n}(x, x ; h)=c h^{-1}+o\left(h^{-1}\right) .
$$

In the case of the kernel estimator, the function $K_{n}$ in representation (66) satisfies $K_{n}\left(x_{i}, x_{i} ; h\right)=h^{-1} K(0)$. Also, as indicated in Chapter 4, under some regularity conditions, the spline smoother under uniform design can be approximated by the kernel estimator (Silverman, 1984)

$$
f_{n}(x)=(n h)^{-1} \sum_{i=1}^{n} K_{s}\left(h^{-1}\left(x-x_{i}\right)\right) Y_{i}
$$

where $K_{s}$ is the spline kernel given by

$$
K_{s}(u)=\frac{1}{2} \exp \left(-\frac{|u|}{\sqrt{2}}\right) \sin \left(\frac{|u|}{\sqrt{2}}+\frac{\pi}{4}\right)
$$


Therefore, under these conditions, function $K_{n}$ in (66) satisfies $K_{n}\left(x_{i}, x_{i} ; h\right)=h^{-1} K_{s}(0)$. Note that relations (64) and (65) are derived under the assumption of a compactly supported kernel function $K$, which does not hold in the case of the spline kernel $K_{s}$. Nevertheless, it looks plausible that (64) and (65) would continue to hold, because $K_{s}(x)$ decreases fast, namely at an exponential rate, as $|x| \rightarrow \infty$. Projection estimator (63) can be also rewritten in the desired form, as shown in Chapter 2:

$$
\begin{aligned}
\tilde{f}_{n}(x) & =\sum_{k=0}^{N-1} \tilde{c}_{k} \phi_{k}(x)=n^{-1} \sum_{i=1}^{n} Y_{i}\left(\sum_{k=0}^{N-1} \phi_{k}\left(x_{i}\right) \phi_{k}(x)\right) \\
& =n^{-1} \sum_{i=1}^{n} K_{n}\left(x, x_{i} ; N\right) Y_{i},
\end{aligned}
$$

with $K_{n}\left(x, x_{i} ; N\right)=\sum_{k=0}^{N-1} \phi_{k}\left(x_{i}\right) \phi_{k}(x)$. It can be shown that $K_{n}\left(x_{i}, x_{i} ; N\right)=$ $\sum_{k=0}^{N-1} \phi_{k}\left(x_{i}\right) \phi_{k}\left(x_{i}\right)=O(N)$. Consider, for example, the Chebyshev case. Using identity 1.351 .2 of Gradshteyn and Ryzhik (1979), we get for $i=1, \ldots, n$,

$$
\begin{aligned}
\sum_{k=0}^{N-1} t_{k}^{2}\left(x_{i}\right) & =1+\sum_{k=1}^{N-1} 2 \cos ^{2}\left(k \arccos x_{i}\right) \\
& =N+\frac{\cos \left(N y_{i}\right) \sin \left((N-1) y_{i}\right)}{\sin y_{i}}=O(N),
\end{aligned}
$$

where $x_{i}=\cos y_{i}$. In some interesting cases, the optimal value of the parameter $N$ in orthogonal series estimation is related to the optimal value of the bandwidth $h$ in kernel estimation through the relation $h=N^{-1}(1+o(1))$. Then, property (67) is again satisfied.

Thus, under some regularity conditions (see, Härdle, Hall, and Marron, 1988), for the linear estimators discussed in this thesis, the cross-validation and generalized cross-validation selection criteria perform nearly equally well, provided $n$ is sufficiently large. 
Now, assume a fixed design model

$$
Y_{i}=f\left(x_{i}\right)+\varepsilon_{i}, \quad i=1, \ldots, n
$$

with uniform design $\left\{x_{i}\right\}_{i=1}^{n}$, where $x_{i}=(i-1) / n$. Suppose that the kernel estimator $f_{n}(x)=n^{-1} \sum_{i=1}^{n} K_{h}\left(x-x_{i}\right) Y_{i}$, where $K(x)$ is a symmetric probability density with $\int x K(x) d x=0$, is used. Recall that the risk $R(h)$ and its unbiased estimator $\hat{R}(h)$ are given by

$$
R(h)=n^{-1} \sum_{i=1}^{n} \mathbf{E}\left(f\left(x_{i}\right)-f_{n}\left(x_{i}\right)\right)^{2}, \quad \hat{R}(h)=n^{-1} R S S(h)-\sigma^{2}+2 n^{-1} \sigma^{2} \operatorname{tr}\left[\mathbf{S}_{n}\right] .
$$

Define $h_{n}$ and $h_{n}^{*}$ as follows:

$$
h_{n}^{*}=\underset{h}{\operatorname{argmin}} R(h) \quad \text { and } \quad h_{n}=\underset{h}{\operatorname{argmin}} \hat{R}(h)
$$

Also let $\theta_{n}=n^{-1 / 5} h_{n}^{-1}, \theta_{n}^{*}=n^{-1 / 5} h_{n}^{*-1}$, and let $\theta^{*}=\lim _{n \rightarrow \infty} \theta_{n}^{*}$. Then due to Corollary 2.2. from Rice (1984), under mild regularity conditions on $f$ and $K$,

$$
\theta_{n} \stackrel{\mathbf{P}}{\longrightarrow} \theta^{*}, \quad n \rightarrow \infty
$$

This result shows that the difference between the bandwidth minimizing the risk $R(h)$ and the bandwidth minimizing the unbiased risk criterion $\hat{R}(h)$ tends to 0 at a rate of $n^{-1 / 5}$ for kernel estimators in the uniform design case. That is,

$$
n^{-1 / 5}\left(h_{n}^{-1}-h_{n}^{*-1}\right) \stackrel{\mathbf{P}}{\longrightarrow} 0, \quad n \rightarrow \infty .
$$

However, the unbiased risk criterion requires the knowledge of $\sigma^{2}$, which is not usually available in practice. Now, we give arguments showing that $G C V(h)$ and $C V(h)$ are 
asymptotically equivalent to $\hat{R}(h)$ for $h \in H_{n}$, and hence that the above result by Rice applies to both $C V(h)$ and $G C V(h)$, provided $n$ is sufficiently large.

Consider, for example, the $G C V(h)$ criterion given by (60). It follows form (61) that, in the case of the kernel estimator, $G C V(h)$ can be rewritten as

$$
\begin{aligned}
G C V(h) & =n^{-1} R S S(h)\left(n^{-1} \operatorname{tr}\left[\mathbf{I}-\mathbf{S}_{n}\right]\right)^{-2} \\
& =n^{-1} R S S(h)\left(1-(n h)^{-1} K(0)\right)^{-2} \\
& =n^{-1} \operatorname{RSS}(h) \Xi_{G C V}\left((n h)^{-1} K(0)\right),
\end{aligned}
$$

where $\Xi_{G C V}$ is the GCV penalizing function

$$
\Xi_{G C V}(u)=(1-u)^{-2}
$$

with first-order Taylor expansion $\Xi_{G C V}(u)=1+2 u+O\left(u^{2}\right)$ for $u \rightarrow 0$. For any $h \in H_{n},(n h)^{-1} K(0) \rightarrow 0$, as $n \rightarrow \infty$. This allows us to express the following as a Taylor expansion

$$
G C V(h)=n^{-1} R S S(h)\left(1+2(n h)^{-1} K(0)+O_{P}\left((n h)^{-2}\right)\right) .
$$

Therefore, applying (56) and (57),

$$
\begin{aligned}
G C V(h) & =n^{-1} R S S(h)\left(n^{-1} \operatorname{tr}\left[\mathbf{I}-\mathbf{S}_{n}\right]\right)^{-2} \\
& =\left(\hat{R}(h)+\sigma^{2}-2 n^{-1} \sigma^{2} \operatorname{tr}\left[\mathbf{S}_{n}\right]\right)\left(1+2(n h)^{-1} K(0)+O_{P}\left((n h)^{-2}\right)\right) \\
& =\hat{R}(h)+\sigma^{2}+O_{P}\left((n h)^{-1}\right) .
\end{aligned}
$$

In this expansion the second term does not depend on $h$, and the third term is of higher order than the previous terms. So, for large $n$, the value of the bandwidth 
that minimizes $G C V(h)$ will be nearly equal to the value of the bandwidth that minimizes $\hat{R}(h)$. Therefore, due to the result of Rice, for sufficiently large $n$, the bandwidth minimizing $G C V(h)$ for a kernel regression estimator will tend to the optimal bandwidth at the rate of $n^{-1 / 5}$. It can be shown that, under the same assumptions on the model and the estimator, a similar conclusion holds for $C V(h)$ (see, for example, Härdle, 1989, p. 174) 


\section{Conclusion}

Much of the fundamental literature on nonparametric regression estimation is not straightforward and requires the knowledge of various mathematical disciplines at an advanced level. In this thesis, we have reviewed and presented some of the important results on this subject. A combination of theoretical justification with informal discussion and simulations should provide insight into the field of nonparametric regression estimation. We have set out to provide an introduction to this field that we hope will be accessible and interesting for Master's level students. 


\section{Appendix}

\section{Useful Inequality}

Minkowski's Inequality for Integrals: Let $f(x)$ and $g(x)$ be two functions such that $|f(x)|^{p}$ and $|g(x)|^{p}$ are integrable on $[a, b]$, where $p \geq 1$. Then

$$
\left(\int_{a}^{b}|f(x)+g(x)|^{p} d x\right)^{1 / p} \leq\left(\int_{a}^{b}|f(x)|^{p} d x\right)^{1 / p}+\left(\int_{a}^{b}|g(x)|^{p} d x\right)^{1 / p}
$$

\section{Multivariate Identities}

Let $\boldsymbol{W}=\left\{w_{i j}\right\}$ be a symmetric $n \times n$ matrix and let $\boldsymbol{\varepsilon}=\left(\varepsilon_{1}, \ldots, \varepsilon_{n}\right)^{\top}$ be a vector of random variables. Consider the quadratic form in $\varepsilon$

$$
Q=\varepsilon^{T} W \varepsilon
$$

If $\mathrm{E} \varepsilon=\boldsymbol{O}$ and $\mathrm{E} \varepsilon \varepsilon^{T}=\Sigma$, then

$$
\mathrm{E} Q=\operatorname{tr}[\Sigma \boldsymbol{W}]
$$

\section{Differentiation Rules}

Let $\mathbf{a}=\left(a_{1}, \ldots, a_{n}\right)^{\top}$ and $\mathbf{b}=\left(b_{1}, \ldots, b_{n}\right)^{\top}$ be two vectors and let $\mathbf{A}$ be a symmetric $n \times n$ matrix. Then, using the notation $\frac{d}{d \mathbf{b}}=\left(\frac{d}{d b_{1}}, \ldots, \frac{d}{d b_{n}}\right)^{\top}$,

$$
\frac{d}{d \mathbf{b}}\left(\mathbf{a}^{\top} \mathbf{b}\right)=\frac{d}{d \mathbf{b}}\left(\mathbf{b}^{\top} \mathbf{a}\right)=\mathbf{a}
$$




$$
\frac{d}{d \mathbf{b}}\left(\mathbf{b}^{\top} \mathbf{A b}\right)=2 \mathbf{A b}
$$

Proof: Note that $\mathbf{a}^{\top} \mathbf{b}$ is a scalar equal to

$$
\mathbf{a}^{\top} \mathbf{b}=\mathbf{b}^{\top} \mathbf{a}=\sum_{i=1}^{n} a_{i} b_{i}
$$

For $k=1, \ldots, n$,

$$
\frac{d}{d b_{k}} \sum_{i=1}^{n} a_{i} b_{i}=a_{k}
$$

Thus (B3) holds true.

Next,

$$
\mathbf{b}^{\top} \mathbf{A} \mathbf{b}=\sum_{i=1}^{n} \sum_{j=1}^{n} b_{i} a_{i j} b_{j}
$$

Using the symmetry of $\mathbf{A}$, for $k=1, \ldots, n$,

$$
\frac{d}{d b_{k}} \sum_{i=1}^{n} \sum_{j=1}^{n} b_{i} a_{i j} b_{j}=2 \sum_{i=1}^{n} a_{i k} b_{k}
$$

- the $i$ th component of the vector $2 \mathrm{Ab}$. Thus (B4) also holds. 


\section{References}

[1] L. M. Artiles (2001). Adaptive Minimax Estimation in Classes of Smooth Functions. Ph.D. Thesis, Utrecht University, Utrecht.

[2] L. M. Artiles and B. Y. Levit (2003). Adaptive estimation of analytic functions on an interval. Math. Methods Statist., 12, 62-94.

[3] J. K. Benedetti (1977). On the nonparametric estimation of regression functions. J. Roy. Stat. Soc. B, 39, 248-253.

[4] I. N. Bronshteyn, K. A. Semendyayev, G. Musiol, H. Muehlig (1998). Handbook of Mathematics. Springer, Berlin.

[5] C. K. Carter, G. K. Eagleson, B. W. Silverman (1992). A comparison of the Reinsch and Speckman splines. Biometrika, 79, 81-91.

[6] N. N. Čencov (1962). Statistical Decision Rules and Optimal Inference. American Mathematical Society, Providence, R.I.

[7] J. Cho (2007). Optimal Design in Regression and Spline Smoothing. Ph.D. Thesis, Queen's University, Kingston.

[8] D. D. Cox (1983). Asymptotics for M-type smoothing splines. Ann. Statist., 11, 530-551.

[9] P. Craven and G. Wahba (1979). Smoothing noisy data with spline functions. Numer. Math., 31, 377-403.

[10] C. De Boor (1963). Best approximation properties of spline functions of odd degree. Journal of Mathematics and Mechanics, 12, 747-750.

[11] C. De Boor (1978). A Practical Guide to Splines. Springer-Verlag, New York.

[12] A. Demmler and C. Reinsch (1975). Oscillation matrices with spline smoothing. Numer. Math., 24, 375-382.

[13] P. Deufhard and A. Hohmann (2000). Numerical Analysis in Modern Scientific Computing. Springer, New York.

[14] S. Efromovich (1996). On nonparametric regression for iid observations in a general setting. Ann. Statist., 24, 1126-1144.

[15] E. Engel (1857). Die vorherrschenden Gewerbszweige in den Gerichtsämtern mit Beziehung auf die Productions- und Consumptionsverhältnisse des Königreichs Sachsen. Z. Statist. Bureaus K. Sächs. Ministerium Innern, 8(9), 153-182.

[16] R. L. Eubank (1999). Nonparametric Regression and Spline Smoothing. Marcel Dekker, New York.

[17] J. Fan and I. Gijbels (1996). Local Polynomial Modeling and its Applications. Chapman and Hall, London.

[18] G. M. Fikhtengolts (1963). Course of Differential and Integral Calculus. Izdatel'stvo FizikoMatematicheskoy Literatury, Moscow. 
[19] F. Galton (1886). Regression towards mediocrity in hereditary stature. Journal of the Anthropological Institute, 15, 246-263.

[20] T. Gasser and H. G. Müller (1979). Kernel estimation of regression functions. Smoothing Techniques for Curve Estimation. Lecture Notes in Mathematics. Springer, New York.

[21] T. Gasser, H. G. Müller, W. Köhler, L. Molinari, A. Prader (1984). Nonparametric regression analysis of growth curves. Ann. Statist., 12, 210-229.

[22] Y. K. Golubev, B. Y. Levit, A.B. Tsybakov (1996). Asymptotic efficient estimation of analytic functions in Gaussian noise. Bernoulli, 2, 167-181.

[23] Y. K. Golubev and B. Y. Levit (1996). Asymptotically efficient estimation for analytic distributions. Math. Methods Statist. 5(3) 357-368.

[24] G. Golubev and M. Nussbaum (1990). A risk bound in Sobolev class regression. Ann. Statist., $18,758-778$.

[25] I. S. Gradshteyn and I. M. Ryzhik (1979). Tables of Integrals, Series, and Products. Academic Press, New York.

[26] T. N. E. Greville (1969). Theory and Application of Spline Functions. Academic Press, New York.

[27] E. Guerre and A. B. Tsybakov (1998). Exact asymptotic minimax constants for the estimation of analytic functions in $L_{p}$. Probab. Theory Relat. Fields, 112, 33-51.

[28] P. Hall, W. Härdle, J.S. Marron (1988). How far are automatically chosen regression smoothing parameters from their optimum? JASA, 83, 86-95.

[29] W. Härdle (1989). Applied Nonparamertic Regression. Cambridge University Press, Cambridge.

[30] W. Härdle, M. Müller, S. Sperlich, A. Werwatz (2000). Nonparametric and Semiparametric Models. Springer-Verlag, New York.

[31] I. A. Ibragimov and R. Z. Hasminskii (1983). Estimation of distribution density. J. Soviet. Math., 25, 40-57.

[32] I. A. Ibragimov and R. Z. Hasminskii (1984). On nonparametric estimation of a linear functional in Gaussian white noise. Prob. Theory Appl., 29, 18-32.

[33] M. H. Kutner (2005). Applied Linear Statistical Models. McGraw-Hill Irwin, Boston.

[34] B. Y. Levit and N. A. Stepanova (2004). Efficient estimation of multivariate analytic functions in cube-like domains. Math. Methods Statist., 13, 253-281.

[35] H. G. Müller (1988). Nonparametric Analysis of Longitudinal Data. Lecture Notes in Statistics. Springer, New York.

[36] E. A. Nadaraya (1964). On estimating regression. Theor. Probab. Appl., 9, 141-142.

[37] S. Nikolskii (1975). Approximations of Functions of Several Variables and Imbedding Theorems. Springer-Verlag, Berlin Heidelberg New York. 
[38] M. Nussbaum (1985). Spline smoothing in regression models and asymptotic efficiency in $L_{2}$. Ann. Statist., 13, 984-997.

[39] E. Parzen (1958). On asymptotically efficient estimates of the spectral density function of a stationary time series. J. Roy. Stat. Soc. Ser. B , 20, 303-322.

[40] E. Parzen (1962). On estimation of a probability density function and mode. Ann. Statist., 33, 1065-1076.

[41] M. S. Pinsker (1980). Optimal filtration of square-intagrable signals in Gaussian noise. Problems of Information Transmission, 16, 120-133.

[42] M. B. Priestly and M. T. Chao (1972). Nonparametric function fitting. J. Roy. Stat. Soc. B Met., 34, 385-392.

[43] Y. V. Prokhorov (1999). Probability and Mathematical Statistics Encyclopedia. Bol'shaya Rossiyskaya Entsiklopedia, Moscow.

[44] C. Reinsch (1967). Smoothing by spline functions. Numer. Math., 10, 177-183.

[45] J. Rice (1984). Bandwidth choice for nonparametric regression. Ann. Statist., 12, 1215-1230.

[46] M. Rosenblatt (1956). Some regression problems in time series analysis. Proceedings of the Third Berkley Symposium on Mathematical Statistics and Probability, 1, 165-168.

[47] D. Ruppert and M. P. Wand (1994). Multivariate weighted least squares regression. Ann. Statist., 22, 1346-1370.

[48] I. J. Schoenberg (1946). Contributions to the problem of approximation of equidistant data by analytic functions. Q. Appl. Math., 4, 45-99 and 112-141.

[49] W. J. Shih and S. Weisberg (1986). Assessing influence in multiple linear regression with incomplete data. Technometrics, 28, 231-239.

[50] B.W. Silverman (1984). Spline smoothing: the equivalent variable kernel method. Ann. Statist., 12, 898-916.

[51] P. Speckman (1985). Spline smoothing and optimal rates of convergence in nonparametric regression models. Ann. Statist., 13, 970-983.

[52] P. K. Suetin (1979). Classical Orthogonal Polynomials. Nauka, Moscow. (In Russian).

[53] A. F. Timan (1994). Theory of Approximation of Functions of a Real Variable. Dover Publications, New York.

[54] A. Vretblad (2003). Fourier Analysis and its Applications. Springer, New York.

[55] G. Wahba (1975). Optimal convergence properties of variable knot, kernel and orthogonal series methods for density estimation. Ann. Statist., 3, 15-29.

[56] G. Wahba (1978). Improper priors, spline smoothing and the problem of guarding against model errors in regression. J. Roy. Stat. Soc. Ser. B , 40, 364-372.

[57] G. G. Walter (1977). Properties of Hermite series estimation of probability density. Ann. Statist., 5, 1258-1264. 
[58] L. Wasserman (2006). All of Nonparametric Statistics. Springer, New York.

[59] G. S. Watson (1964). Smoothing regression analysis. Sankhyā Series A, 26, 359-372.

[60] E. T. Whittaker (1923). On a new method of graduation. P. Edinbugh Math. Soc., 41, 63-75. 